\title{
Palladium-Catalyzed 1,3-Difunctionalization using Terminal Al- kenes with Alkenyl Nonaflates and Aryl Boronic Acids
}

\author{
Matthew S. McCammant, Takashi Shigeta, and Matthew S. Sigman* \\ Department of Chemistry, University of Utah, 315 South 1400 East, Salt Lake City, Utah 84112, United States
}

Table of Contents

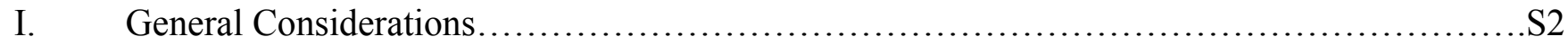

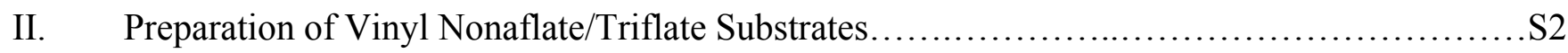

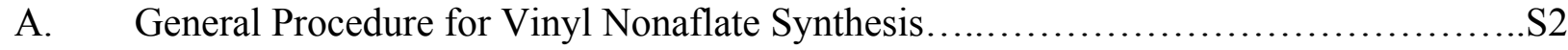

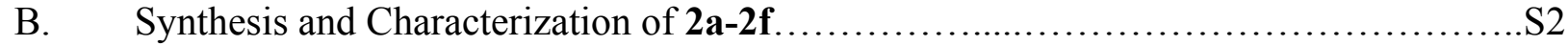

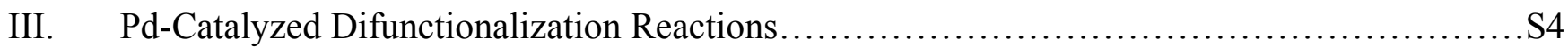

A. General Procedure for Reaction Optimization..................................S4

B. Tabular Summary of Reaction Optimization Studies............................... 5

C. Optimized General Procedure.................................................. 5

D. Synthesis and Characterization of $\mathbf{4 a - 4 v \ldots \ldots \ldots \ldots \ldots \ldots \ldots \ldots \ldots \ldots \ldots \ldots \ldots \ldots \ldots \ldots \ldots \ldots \ldots \ldots}$

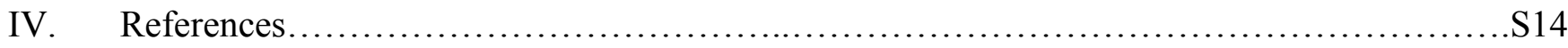

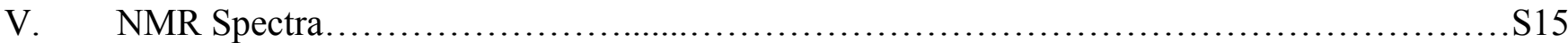




\section{General Considerations}

Anhydrous dimethylformamide (DMF) was purchased from Sigma-Aldrich and stored over activated 3 $\AA$ molecular sieves $(3 \AA \mathrm{MS}) . \mathrm{Pd}_{2}(\mathrm{dba})_{3} \cdot \mathrm{CHCl}_{3}$ was synthesized according to known procedure. ${ }^{1}$ Unless otherwise noted all reagents and solvents were purchased from Sigma-Aldrich, Frontier Scientific, Combi-Blocks, Oakwood Chemical, or TCI and used without further purification. ${ }^{1} \mathrm{H}$ NMR spectra were obtained at $500 \mathrm{MHz}$ or $400 \mathrm{MHz}$. Chemical shifts are reported in ppm and referenced to the $\mathrm{CHCl}_{3}$ singlet at $7.26 \mathrm{ppm}$. The abbreviations $\mathrm{s}, \mathrm{d}, \mathrm{t}, \mathrm{q}$, quint, sext, dd, ddd, app. dt, dt, and $\mathrm{m}$ stand for the resonance multiplicities singlet, doublet, triplet, quartet, quintet, sextet, doublet of doublets, doublet of doublets of doublets, apparent doublet of triplet, doublet of triplet, and multiplet, respectively. ${ }^{13} \mathrm{C}$ NMR spectra were obtained at 126 or $100 \mathrm{MHz}$ and referenced to the centerline of the $\mathrm{CDCl}_{3}$ triplet at 77.23 ppm. Flash chromatography was performed using Silicycle SiliaFlash F60 silica gel (230-400 mesh). Silver nitrate impregnated silica gel $(10 \% \mathrm{w} / \mathrm{w})$ for flash chromatography was prepared by dissolving $\mathrm{AgNO}_{3}$ in acetonitrile, and then mixing with silica gel. ${ }^{2-3}$ This was concentrated in vacuo, and then further dried in a $150{ }^{\circ} \mathrm{C}$ oven for three hours. Silver impregnated silica gel was stored in the absence of light until use. Gas Chromatography (GC) separations were performed with an HP6890 GC with a flame ionization detector equipped with a DB-5 column using a 50:1 split. IR spectra were recorded using a Thermo Nicolet FTIR. HRMS data were obtained on a Waters LCP Premier XE instrument by ESI/TOF. LR GC-MS data were obtained on an Agilent Technologies 5975C VL MSD instrument.

\section{Preparation of Vinyl Nonaflate/Triflate Substrates}

\section{A. General Procedure for Vinyl Nonaflate Synthesis}

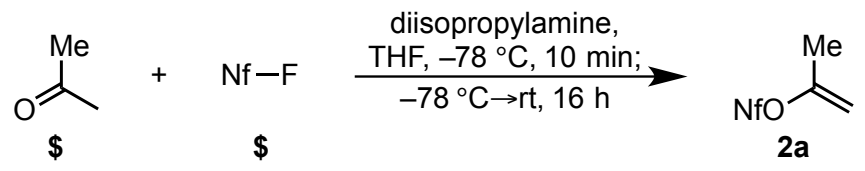

To an oven dried $250 \mathrm{~mL}$ round bottom flask under an $\mathrm{N}_{2}$ atmosphere was added $3.08 \mathrm{~mL}(22 \mathrm{mmol}$, 1.1 equiv) freshly distilled diisopropylamine and THF $(80 \mathrm{~mL})$. The solution was cooled to $-78^{\circ} \mathrm{C}$, and then $8.0 \mathrm{~mL}$ ( $2.5 \mathrm{M}$ in hexanes, $20 \mathrm{mmol}, 1.0$ equiv) $n$-BuLi was added dropwise. The reaction mixture was stirred for 10 minutes, and then $1.47 \mathrm{~mL}(20 \mathrm{mmol}, 1.0$ equiv) acetone (freshly distilled from $\mathrm{CaSO}_{4}$ ) was added dropwise. The mixture was stirred for 10 minutes at $-78{ }^{\circ} \mathrm{C}$, then warmed to room temperature. After 30 minutes the reaction flask was cooled back to $-78{ }^{\circ} \mathrm{C}$, and then $4.31 \mathrm{~mL}(24$ mmol, 1.2 equiv) perfluoro-1-butanesulfonyl fluoride was added. The reaction was stirred for 16 hours while slowly warming to room temperature. After completion, the reaction was quenched with a saturated solution of $\mathrm{NaHCO}_{3}(100 \mathrm{~mL})$ and transferred to a separatory funnel with $\mathrm{Et}_{2} \mathrm{O}(50 \mathrm{~mL})$. The organic layer was washed with a $1 \mathrm{M}$ solution of $\mathrm{NaOH}(4 \times 50 \mathrm{~mL})$, water $(2 \times 50 \mathrm{~mL})$, and brine $(1 \times 50$ $\mathrm{mL})$, then dried over sodium sulfate. The dried organic mixture was concentrated in vacuo and purified by silica gel flash chromatography.

\section{B. Synthesis and Characterization of $2 \mathrm{a}-2 \mathrm{f}$}

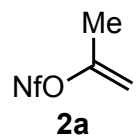

prop-1-en-2-yl nonafluorobutane-1-sulfonate (2a). The general procedure was followed using 3.08 $\mathrm{mL}(22 \mathrm{mmol})$ diisopropylamine in THF $(80 \mathrm{~mL}), 8.0 \mathrm{~mL}(2.5 \mathrm{M}$ in hexanes, $20 \mathrm{mmol}) n$-BuLi, 1.47 $\mathrm{mL}(20 \mathrm{mmol})$ acetone (freshly distilled from $\left.\mathrm{CaSO}_{4}\right)$, and $4.31 \mathrm{~mL}(24 \mathrm{mmol})$ perfluoro-1- 
butanesulfonyl fluoride. Purification by silica gel flash chromatography (99:1 pentane:Et ${ }_{2} \mathrm{O}$, silica gel was neutralized using a $1 \%$ solution of $\left.\mathrm{NH}_{4} \mathrm{OH}\right)$ led to the isolation of $\mathbf{2 a}$ as a colorless oil $(2.45 \mathrm{~g}$, $36 \%), \mathrm{R}_{f}=0.23$ (99:1 pentane: $\mathrm{Et}_{2} \mathrm{O}$, visualized by $\mathrm{KMnO}_{4}$ stain). ${ }^{1} \mathrm{H} \mathrm{NMR}\left(\mathrm{CDCl}_{3}, 400 \mathrm{MHz}\right): \delta 5.09$ $(\mathrm{d}, J=3.2 \mathrm{~Hz}, 1 \mathrm{H}), 4.95(\mathrm{~s}, 1 \mathrm{H}), 2.10(\mathrm{~s}, 3 \mathrm{H}) ;{ }^{13} \mathrm{C} \mathrm{NMR}\left(\mathrm{CDCl}_{3}, 126 \mathrm{MHz}\right): \delta 153.5,119.0-106.0(\mathrm{~m}$, 4C), 105.7, 20.4; FTIR (thin film): 2923, 1653, 1457, 1092, 1016, $795 \mathrm{~cm}^{-1}$; LR GC-MS $m / z$ calculated for $\mathrm{C}_{7} \mathrm{H}_{5} \mathrm{~F}_{9} \mathrm{O}_{3} \mathrm{~S}: 339.98$, found 340.00 .

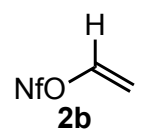

vinyl nonafluorobutane-1-sulfonate (2b). A previously reported procedure was used for the synthesis of $\mathbf{2 b}$ from tetrahydrofuran. ${ }^{4}$ Purification by short path distillation led to the isolation of $\mathbf{2 b}$ as a colorless oil (7.24 g, 50\%). ${ }^{1} \mathrm{H}$ NMR $\left(\mathrm{CDCl}_{3}, 400 \mathrm{MHz}\right): \delta 6.77(\mathrm{dd}, J=13.5,5.6 \mathrm{~Hz}, 1 \mathrm{H}), 5.26(\mathrm{dd}, J=$ 13.2, $3.6 \mathrm{~Hz}, 1 \mathrm{H}), 5.00(\mathrm{~m}, 1 \mathrm{H}) ;{ }^{13} \mathrm{C} \mathrm{NMR}\left(\mathrm{CDCl}_{3}, 126 \mathrm{MHz}\right): \delta 142.8,121.0-104.2(\mathrm{~m}, 4 \mathrm{C}), 104.2$; FTIR (thin film): 1646, 1197, 1142, 1085, 960, 895, $755 \mathrm{~cm}^{-1}$; LR GC-MS $\mathrm{m} / z$ calculated for $\mathrm{C}_{6} \mathrm{H}_{3} \mathrm{~F}_{9} \mathrm{O}_{3} \mathrm{~S}: 325.97$, found 326.00 .

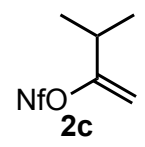

3-methylbut-1-en-2-yl nonafluorobutane-1-sulfonate (2c). The general procedure was followed using $1.54 \mathrm{~mL}$ (11 mmol) diisopropylamine in THF $(40 \mathrm{~mL}), 4.0 \mathrm{~mL}(2.5 \mathrm{M}$ in hexanes, $10 \mathrm{mmol}) n^{-}$ $\mathrm{BuLi}, 1.05 \mathrm{~mL}(10 \mathrm{mmol})$ methyl isopropyl ketone (freshly distilled from $\left.\mathrm{CaSO}_{4}\right)$, and $2.16 \mathrm{~mL}(12$ mmol) perfluoro-1-butanesulfonyl fluoride. Purification by silica gel flash chromatography (19:1 hexanes:ethyl acetate, silica gel was neutralized using a $1 \%$ solution of $\mathrm{NH}_{4} \mathrm{OH}$ ) led to the isolation of 2c as a colorless oil $(1.20 \mathrm{~g}, 50 \%), \mathrm{R}_{f}=0.50$ (19:1 hexanes:ethyl acetate, visualized by $\mathrm{KMnO}_{4}$ stain). ${ }^{1} \mathrm{H}$ NMR $\left(\mathrm{CDCl}_{3}, 500 \mathrm{MHz}\right): \delta 5.09(\mathrm{~d}, J=4.0 \mathrm{~Hz}, 1 \mathrm{H}), 4.94(\mathrm{dd}, J=3.9,0.8 \mathrm{~Hz}, 1 \mathrm{H}), 2.56(\mathrm{sept}, J=$ $7.0 \mathrm{~Hz}, 1 \mathrm{H}), 1.16(\mathrm{~d}, J=7.0 \mathrm{~Hz}, 6 \mathrm{H}) ;{ }^{13} \mathrm{C} \mathrm{NMR}\left(\mathrm{CDCl}_{3}, 126 \mathrm{MHz}\right): \delta 162.5,120.0-106.0(\mathrm{~m}, 4 \mathrm{C})$, 101.9, 33.3, 20.0; FTIR (thin film): 1418, 1200, 1142, 933, 798, $739 \mathrm{~cm}^{-1}$; LR GC-MS $m / z$ calculated for $\mathrm{C}_{9} \mathrm{H}_{9} \mathrm{~F}_{9} \mathrm{O}_{3} \mathrm{~S}: 368.01$, found 368.00 .

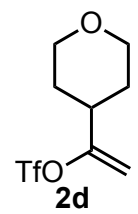

1-(tetrahydropyran-4-yl)vinyl trifluoromethanesulfonate (2d). A previously reported procedure was used for the synthesis of $\mathbf{2 d}$ from 4-acetyltetrahydropyran. ${ }^{5}$ Purification by silica gel flash chromatography (9:1 hexanes:ethyl acetate, silica gel was neutralized using a 1\% solution of $\mathrm{NH}_{4} \mathrm{OH}$ ) led to the isolation of $\mathbf{2 d}$ as a colorless oil $(690 \mathrm{mg}, 66 \%), \mathrm{R}_{f}=0.21$ (9:1 hexanes:ethyl acetate, visualized by $\mathrm{KMnO}_{4}$ stain). ${ }^{1} \mathrm{H} \mathrm{NMR}\left(\mathrm{CDCl}_{3}, 400 \mathrm{MHz}\right): \delta 5.15(\mathrm{~d}, J=4.0 \mathrm{~Hz}, 1 \mathrm{H}), 4.95(\mathrm{~d}, J=4.0$ $\mathrm{Hz}, 1 \mathrm{H}), 4.03(\mathrm{dd}, J=11.8,3.6 \mathrm{~Hz}, 2 \mathrm{H}), 3.42(\mathrm{dt}, J=12.0,1.6 \mathrm{~Hz}, 2 \mathrm{H}), 2.48(\mathrm{~m}, 1 \mathrm{H}), 1.85(\mathrm{~d}, J=12.8$ $\mathrm{Hz}, 2 \mathrm{H}), 1.59(\mathrm{~m}, 2 \mathrm{H}) ;{ }^{13} \mathrm{C} \mathrm{NMR}\left(\mathrm{CDCl}_{3}, 126 \mathrm{MHz}\right): \delta 159.6,118.6(\mathrm{q}, J=320), 103.0,67.4,39.7$, 30.1; FTIR (thin film): 2958, 1414, 1124, 1090, 909, 858, $720 \mathrm{~cm}^{-1}$; HRMS m/z calculated for $\mathrm{C}_{8} \mathrm{H}_{11} \mathrm{~F}_{3} \mathrm{O}_{4} \mathrm{SNa}[\mathrm{M}+\mathrm{Na}]^{+}:$283.0228, found 283.0240.

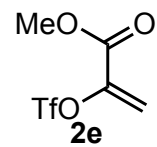

methyl 2-(((trifluoromethyl)sulfonyl)oxy)acrylate (2e). A previously reported procedure was used for the synthesis of 2e from methyl pyruvate. ${ }^{6}$ Purification by silica gel flash chromatography (99:1 
pentane:Et $\mathrm{E}_{2} \mathrm{O}$, silica gel was neutralized using a $1 \%$ solution of $\mathrm{NH}_{4} \mathrm{OH}$ ) led to the isolation of $\mathbf{2 e}$ as a colorless oil $(260 \mathrm{mg}, 22 \%), \mathrm{R}_{f}=0.13$ (99:1 pentane:Et ${ }_{2} \mathrm{O}$, visualized by $\mathrm{KMnO}_{4}$ stain). ${ }^{1} \mathrm{H}$ NMR $\left(\mathrm{CD}_{2} \mathrm{Cl}_{2}, 400 \mathrm{MHz}\right): \delta 6.34(\mathrm{~d}, J=3.6 \mathrm{~Hz}, 1 \mathrm{H}), 5.87(\mathrm{~d}, J=3.6 \mathrm{~Hz}, 1 \mathrm{H}), 3.88(\mathrm{~s}, 3 \mathrm{H}) ;{ }^{13} \mathrm{C} \mathrm{NMR}$ $\left(\mathrm{CD}_{2} \mathrm{Cl}_{2}, 100 \mathrm{MHz}\right): \delta 196.2,160.7,144.2,119.1(\mathrm{q}, J=320), 118.3$; FTIR (thin film): 1747, 1646, $1425,1129,946,853,719 \mathrm{~cm}^{-1}$; HRMS $\mathrm{m} / z$ calculated for $\mathrm{C}_{5} \mathrm{H}_{5} \mathrm{~F}_{3} \mathrm{O}_{5} \mathrm{SNa}[\mathrm{M}+\mathrm{Na}]^{+}: 256.9707$, found 256.9711.

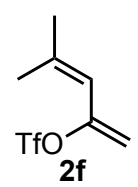

4-methylpenta-1,3-dien-2-yl trifluoromethanesulfonate (2f). A previously reported procedure was used for the synthesis of $\mathbf{2 f}$ from 4-methyl-3-penten-2-one (freshly distilled from $\mathrm{CaSO}_{4}$ ). ${ }^{5}$ Purification by silica gel flash chromatography (100\% hexanes $\rightarrow 99: 1$ hexanes:ethyl acetate) led to the isolation of 2f as a colorless oil (203 mg, 7\%), $\mathrm{R}_{f}=0.39$ (19:1 hexanes:ethyl acetate, visualized by $\mathrm{KMnO}_{4}$ ). Following isolation by silica gel flash chromatography, the material began to decompose and was used immediately as a substrate.

\section{Pd-Catalyzed Difunctionalization Reactions}

\section{A. General Procedure for Reaction Optimization}

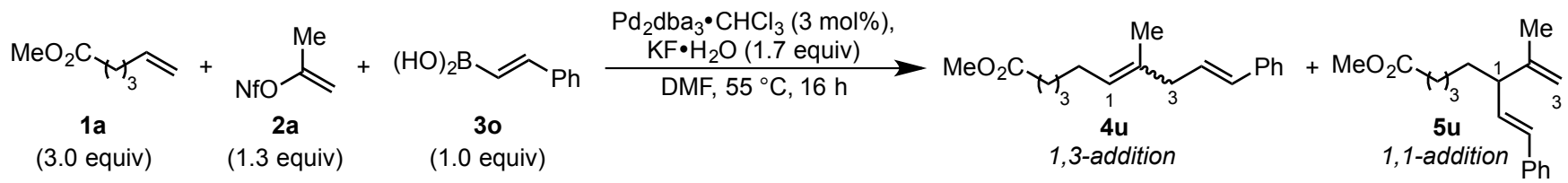

To an oven dried $5 \mathrm{~mL}$ vial were added $16 \mathrm{mg}\left(0.17 \mathrm{mmol}, 1.7\right.$ equiv) of $\mathrm{KF} \bullet 2 \mathrm{H}_{2} \mathrm{O}, 15 \mathrm{mg}(0.1 \mathrm{mmol}$, 1.0 equiv) of $(E)$-(2-phenylvinyl)boronic acid 3o, and $3.1 \mathrm{mg}(3.0 \mathrm{~mol} \%)$ of $\mathrm{Pd}_{2} \mathrm{dba}_{3} \bullet \mathrm{CHCl}_{3}$. The vial was equipped with a PTFE-lined stirbar and flushed with $\mathrm{N}_{2}$ before being sealed with a septum cap. To the solids was added a solution of $44 \mathrm{mg}(0.13 \mathrm{mmol}, 1.3 \mathrm{equiv})$ of $\mathbf{2 a}$ and $42 \mu \mathrm{L}(0.3 \mathrm{mmol}, 3.0$ equiv) of methyl 5-hexenoate 1a in $2.0 \mathrm{~mL}$ of DMF. The mixture was stirred for $16 \mathrm{~h}$ at $55^{\circ} \mathrm{C}$. After completion, $\mathrm{a} \sim 200 \mu \mathrm{L}$ aliquot of the reaction mixture was removed via syringe and filtered through a silica plug, eluting with ethyl acetate. The mixture was analyzed by GC. Yields were calculated using a response factor $\left({ }^{1} \mathrm{H}\right.$ NMR spectroscopy was used to measure the response factor to account for varying detector response) and 2-methoxynaphthalene as an internal standard. 
B. Tabular Summary of Reaction Optimization Studies

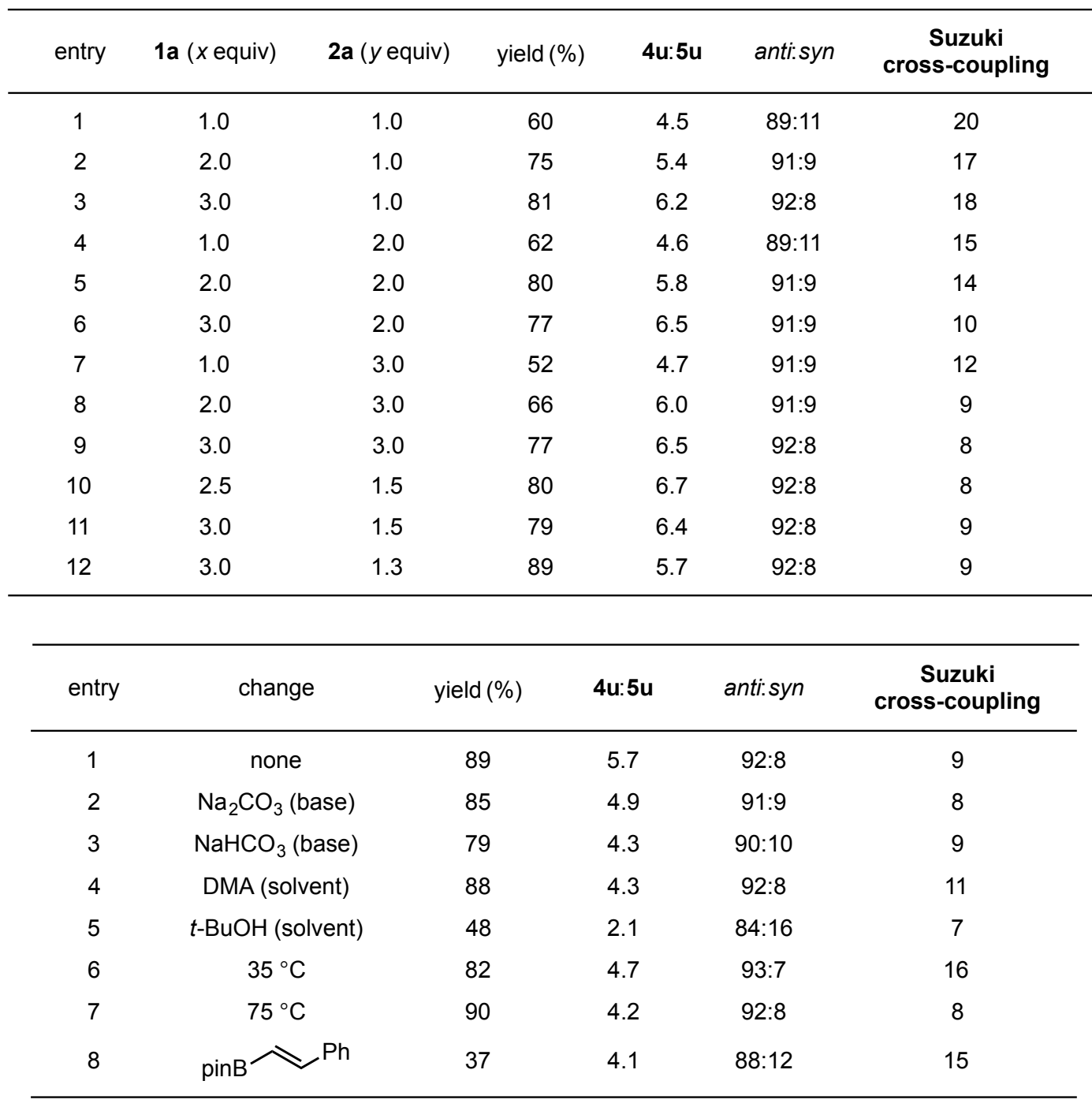

\section{Optimized General Procedure}

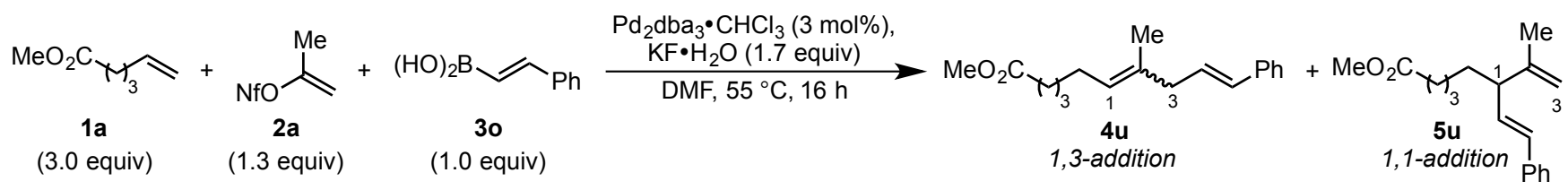

To an oven dried $10 \mathrm{~mL}$ Schlenk flask were added $80 \mathrm{mg}(0.85 \mathrm{mmol}, 1.7$ equiv $)$ of $\mathrm{KF} \bullet 2 \mathrm{H}_{2} \mathrm{O}, 74 \mathrm{mg}$ (0.5 mmol, 1.0 equiv) of (E)-(2-phenylvinyl)boronic acid 3o, and $16 \mathrm{mg}(3.0 \mathrm{~mol} \%)$ of $\mathrm{Pd}_{2} \mathrm{dba}_{3} \bullet \mathrm{CHCl}_{3}$. The flask was equipped with a PTFE-lined stirbar and glass joints, and then was evacuated with house vacuum and flushed with $\mathrm{N}_{2}$ three times. To the solids were added $8 \mathrm{~mL}$ of DMF and a solution of 221 $\mathrm{mg}$ ( $0.65 \mathrm{mmol}, 1.3$ equiv) of $\mathbf{2 a}$ and $0.21 \mathrm{~mL}$ (1.5 mmol, 3.0 equiv) of methyl 5-hexenoate $1 \mathbf{a}$ in 2.0 $\mathrm{mL}$ of DMF. The mixture was stirred for $8 \mathrm{~h}$ at $55^{\circ} \mathrm{C}$. After completion, the mixture was diluted with methyl tert-butyl ether and filtered through a Celite plug. The organics were washed with water $(3 \times 10$ $\mathrm{mL})$ and brine $(1 \times 10 \mathrm{~mL})$, then dried over sodium sulfate and concentrated in vacuo. Products were purified by silica gel flash chromatography as noted below. Yields represent a mixture of stereo and regioisomers. Enriched stereo- and regioisomer fractions were isolated using $\mathrm{AgNO}_{3}$ impregnated silica 
gel flash chromatography. NMR analysis was used to verify the identity of isomers. GC analysis was used to determine isomeric ratios of the crude product mixture.

\section{Synthesis and Characterization of $4 a-4 v$}

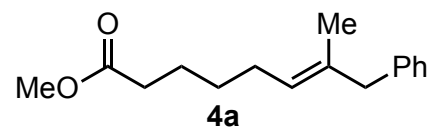

methyl $(\boldsymbol{E})$-7-methyl-8-phenyloct-6-enoate $((\boldsymbol{E})-4 a)$. The general procedure was followed using 80 $\mathrm{mg}$ of $\mathrm{KF} \bullet 2 \mathrm{H}_{2} \mathrm{O}(0.85 \mathrm{mmol}), 61 \mathrm{mg}$ of phenylboronic acid $\mathbf{3 a}(0.5 \mathrm{mmol}), 16 \mathrm{mg}$ of $\mathrm{Pd}_{2} \mathrm{dba}_{3} \bullet \mathrm{CHCl}_{3}$ (3.0 mol\%), $8 \mathrm{~mL}$ of DMF, and a solution of $221 \mathrm{mg}$ of $2 \mathbf{2}(0.65 \mathrm{mmol})$ and $0.21 \mathrm{~mL}$ of methyl 5hexenoate 1a $(1.5 \mathrm{mmol})$ in $2.0 \mathrm{~mL}$ of DMF. Purification by silica gel flash chromatography (19:1 hexanes:ethyl acetate) led to the isolation of $\mathbf{4 a}$ and $\mathbf{5 a}$ as a colorless oil (106 $\mathrm{mg}, 86 \%$ as a 7.1:0.70:1.0 mixture of $(E)-4 a:(Z)-4 a: 5 a$ isomers respectively), isomeric ratios were determined by $\mathrm{GC}, \mathrm{R}_{f}=0.26$ (19:1 hexanes:ethyl acetate, visualized by $254 \mathrm{~nm}$ light and PMA stain). ${ }^{1} \mathrm{H}$ NMR $\left(\mathrm{CDCl}_{3}, 500 \mathrm{MHz}\right): \delta$ $7.28(\mathrm{~m}, 2 \mathrm{H}), 7.17(\mathrm{~m}, 3 \mathrm{H}), 5.24(\mathrm{t}, J=7.1 \mathrm{~Hz}, 1 \mathrm{H}), 3.67(\mathrm{~s}, 3 \mathrm{H}), 3.28(\mathrm{~s}, 2 \mathrm{H}), 2.32(\mathrm{t}, J=7.6 \mathrm{~Hz}, 2 \mathrm{H})$, $2.04(\mathrm{q}, J=7.6 \mathrm{~Hz}, 2 \mathrm{H}), 1.64(\mathrm{~m}, 2 \mathrm{H}), 1.53$ (s, 3H), 1.40 (pent, $J=7.6 \mathrm{~Hz}, 2 \mathrm{H})$; (The stereochemistry was confirmed by NOESY1D NMR spectroscopy); The following signals can be assigned to $(Z)-4 a$ : ${ }^{1} \mathrm{H}$ $\operatorname{NMR}\left(\mathrm{CDCl}_{3}, 500 \mathrm{MHz}\right): \delta 5.30(\mathrm{t}, J=6.8 \mathrm{~Hz}, 1 \mathrm{H}), 3.36(\mathrm{~s}, 2 \mathrm{H}), 2.15(\mathrm{q}, J=8.0 \mathrm{~Hz}, 2 \mathrm{H}) ;{ }^{13} \mathrm{C} \mathrm{NMR}$ $\left(\mathrm{CDCl}_{3}, 126 \mathrm{MHz}\right): \delta 174.5,140.6,135.0,129.0,128.4,126.4,126.1,51.7,46.5,34.2,29.5,27.8,24.8$, 16.0; FTIR (thin film): 2931, 1737, 1602, 1435, 1170, 733, $699 \mathrm{~cm}^{-1}$; HRMS $\mathrm{m} / z$ calculated for $\mathrm{C}_{16} \mathrm{H}_{22} \mathrm{O}_{2} \mathrm{Na}[\mathrm{M}+\mathrm{Na}]^{+}: 269.1517$, found 269.1516 .

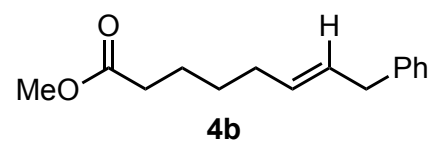

methyl $(\boldsymbol{E})-8$-phenyloct-6-enoate $((\boldsymbol{E})-\mathbf{4 b})$. The general procedure was followed using $80 \mathrm{mg}$ of $\mathrm{KF} \cdot 2 \mathrm{H}_{2} \mathrm{O}(0.85 \mathrm{mmol}), 61 \mathrm{mg}$ of phenylboronic acid $\mathbf{3 a}(0.5 \mathrm{mmol}), 16 \mathrm{mg}$ of $\mathrm{Pd}_{2} \mathrm{dba}_{3} \cdot \mathrm{CHCl}_{3}(3.0$ $\mathrm{mol} \%), 8 \mathrm{~mL}$ of DMF, and a solution of $212 \mathrm{mg}$ of $\mathbf{2 b}(0.65 \mathrm{mmol})$ and $0.21 \mathrm{~mL}$ of methyl 5-hexenoate $1 \mathrm{a}(1.5 \mathrm{mmol})$ in $2.0 \mathrm{~mL}$ of DMF. Purification by silica gel flash chromatography $(97: 3$ hexanes:ethyl acetate) led to the isolation of $\mathbf{4 b}$ and $\mathbf{5 b}$ as a colorless oil (106 $\mathrm{mg}, 91 \%$ as a 10.6:0.61:1.0 mixture of $(E)-\mathbf{4 b}:(Z)-\mathbf{4 b}: 5 \mathbf{b}$ isomers respectively), isomeric ratios were determined by GC, $\mathrm{R}_{f}=0.21$ (19:1 hexanes:ethyl acetate, visualized by $254 \mathrm{~nm}$ light and PMA stain). ${ }^{1} \mathrm{H}$ NMR $\left(\mathrm{CDCl}_{3}, 400 \mathrm{MHz}\right): \delta 7.28(\mathrm{~m}$, 2H), 7.19 (m, 3H), 5.58 (app. dt, $J=15.2,6.4 \mathrm{~Hz}, 1 \mathrm{H}), 5.49$ (app. dt, $J=15.2,6.4 \mathrm{~Hz}, 1 \mathrm{H}), 3.67$ (s, 3H), $3.33(\mathrm{~d}, J=6.4 \mathrm{~Hz}, 2 \mathrm{H}), 2.31(\mathrm{t}, J=7.6 \mathrm{~Hz}, 2 \mathrm{H}), 2.04(\mathrm{q}, J=7.2 \mathrm{~Hz}, 2 \mathrm{H}), 1.64$ (pent, $J=7.6 \mathrm{~Hz}, 2 \mathrm{H})$, 1.41 (pent, $J=7.6 \mathrm{~Hz}, 2 \mathrm{H}$ ); (The stereochemistry was confirmed by NOESY1D NMR spectroscopy); ${ }^{13} \mathrm{C} \mathrm{NMR}\left(\mathrm{CDCl}_{3}, 126 \mathrm{MHz}\right): \delta 174.4,141.2,131.5,129.5,128.7,128.6,126.1,51.7,39.3,34.2,32.3$, 29.1, 24.7; FTIR (thin film): 2926, 1739, 1602, 1435, 1072, 969, 746, $699 \mathrm{~cm}^{-1}$; HRMS $\mathrm{m} / z$ calculated for $\mathrm{C}_{15} \mathrm{H}_{20} \mathrm{O}_{2} \mathrm{Na}[\mathrm{M}+\mathrm{Na}]^{+}: 255.1361$, found 255.1360 .

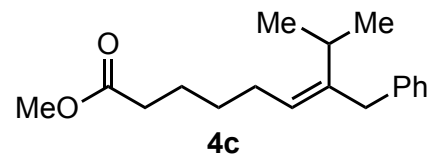

methyl $(Z)$-7-benzyl-8-methylnon-6-enoate $((Z)-4 c)$. The general procedure was followed using 80 $\mathrm{mg}$ of $\mathrm{KF} \bullet 2 \mathrm{H}_{2} \mathrm{O}(0.85 \mathrm{mmol}), 61 \mathrm{mg}$ of phenylboronic acid 3a $(0.5 \mathrm{mmol}), 16 \mathrm{mg}$ of $\mathrm{Pd}_{2} \mathrm{dba}_{3} \bullet \mathrm{CHCl}_{3}$ (3.0 mol\%), $8 \mathrm{~mL}$ of DMF, and a solution of $239 \mathrm{mg}$ of $2 \mathrm{c}(0.65 \mathrm{mmol})$ and $0.21 \mathrm{~mL}$ of methyl 5hexenoate 1a $(1.5 \mathrm{mmol})$ in $2.0 \mathrm{~mL}$ of DMF. Purification by silica gel flash chromatography (49:1 hexanes:ethyl acetate) led to the isolation of $\mathbf{4 c}$ and $\mathbf{5 c}$ as a colorless oil $(72 \mathrm{mg}, 50 \%$ as a 1.2:1.9:1.0 mixture of $(E)-\mathbf{4 c}:(Z)-\mathbf{4 c}: 5 \mathbf{c}$ isomers respectively), isomeric ratios were determined by $\mathrm{GC}, \mathrm{R}_{f}=0.24(19: 1$ hexanes:ethyl acetate, visualized by $254 \mathrm{~nm}$ light and PMA stain). ${ }^{1} \mathrm{H} \mathrm{NMR}\left(\mathrm{CDCl}_{3}, 400 \mathrm{MHz}\right): \delta 7.25$ 
$(\mathrm{m}, 2 \mathrm{H}), 7.16(\mathrm{~m}, 3 \mathrm{H}), 4.85(\mathrm{t}, J=6.8 \mathrm{~Hz}, 1 \mathrm{H}), 3.66(\mathrm{~s}, 3 \mathrm{H}), 3.27(\mathrm{~s}, 2 \mathrm{H}), 2.85(\mathrm{sept}, J=7.2 \mathrm{~Hz}, 1 \mathrm{H})$, $2.30(\mathrm{t}, J=7.6 \mathrm{~Hz}, 2 \mathrm{H}), 2.05$ (q, $J=7.6 \mathrm{~Hz}, 2 \mathrm{H}), 1.62$ (pent, $J=7.6 \mathrm{~Hz}, 2 \mathrm{H}$ ), 1.34 (pent, $J=7.6 \mathrm{~Hz}$, $2 \mathrm{H}), 0.96(\mathrm{~d}, J=6.8 \mathrm{~Hz}, 6 \mathrm{H})$; (The stereochemistry was confirmed by NOESY1D NMR spectroscopy); The following signals can be assigned to $(E)-2 \mathrm{c}:{ }^{1} \mathrm{H}$ NMR $\left(\mathrm{CDCl}_{3}, 400 \mathrm{MHz}\right): \delta 5.35(\mathrm{t}, J=7.2 \mathrm{~Hz}$, $1 \mathrm{H}), 3.42(\mathrm{~s}, 2 \mathrm{H}), 2.14(\mathrm{~m}, 2 \mathrm{H}), 1.40(\mathrm{~m}, 2 \mathrm{H}), 1.07(\mathrm{~d}, J=6.8 \mathrm{~Hz}, 6 \mathrm{H})$; The following signals can be assigned to 5c: ${ }^{1} \mathrm{H}$ NMR $\left(\mathrm{CDCl}_{3}, 400 \mathrm{MHz}\right): \delta 4.94(\mathrm{~d}, J=4.0 \mathrm{~Hz}, 1 \mathrm{H}), 4.88(\mathrm{~d}, J=4.0 \mathrm{~Hz}, 1 \mathrm{H}), 3.64$ $(\mathrm{s}, 3 \mathrm{H}) ;{ }^{13} \mathrm{C} \mathrm{NMR}\left(\mathrm{CDCl}_{3}, 126 \mathrm{MHz}\right): \delta 174.4,144.4,141.4,129.5,128.3,126.2,125.8,51.7,38.5$, 34.2, 29.8, 29.3, 27.3, 24.9, 21.6; FTIR (thin film): 2956, 1737, 1601, 1435, 1361, 1169, 892, $699 \mathrm{~cm}^{-1}$; HRMS $m / z$ calculated for $\mathrm{C}_{18} \mathrm{H}_{26} \mathrm{O}_{2} \mathrm{Na}[\mathrm{M}+\mathrm{Na}]^{+}: 297.1831$, found 297.1830.

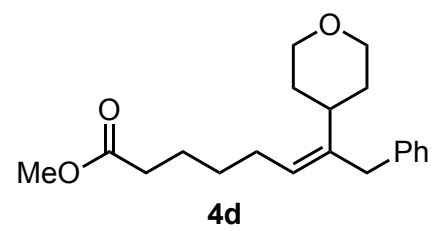

methyl $(Z)-8$-phenyl-7-(tetrahydropyran-4-yl)oct-6-enoate $((Z)-4 d)$. The general procedure was followed using $80 \mathrm{mg}$ of $\mathrm{KF} \cdot 2 \mathrm{H}_{2} \mathrm{O}(0.85 \mathrm{mmol}), 61 \mathrm{mg}$ of phenylboronic acid $3 \mathbf{a}(0.5 \mathrm{mmol}), 16 \mathrm{mg}$ of $\mathrm{Pd}_{2} \mathrm{dba}_{3} \cdot \mathrm{CHCl}_{3}(3.0 \mathrm{~mol} \%), 8 \mathrm{~mL}$ of DMF, and a solution of $169 \mathrm{mg}$ of $2 \mathbf{d}(0.65 \mathrm{mmol})$ and $0.21 \mathrm{~mL}$ of methyl 5-hexenoate 1a $(1.5 \mathrm{mmol})$ in $2.0 \mathrm{~mL}$ of DMF. Purification by silica gel flash chromatography (9:1 hexanes:ethyl acetate) led to the isolation of $\mathbf{4 d}$ and $\mathbf{5 d}$ as a colorless oil (76 $\mathrm{mg}, 48 \%$ as a 1.3:2.0:1.0 mixture of $(E)-\mathbf{4 d}:(Z)-\mathbf{4 d}: 5 \mathbf{d}$ isomers respectively), isomeric ratios were determined by GC, $\mathrm{R}_{f}=0.15$ (9:1 hexanes:ethyl acetate, visualized by $254 \mathrm{~nm}$ light and PMA stain). ${ }^{1} \mathrm{H} \mathrm{NMR}\left(\mathrm{CDCl}_{3}, 400\right.$ $\mathrm{MHz}): \delta 7.25(\mathrm{~m}, 2 \mathrm{H}), 7.17(\mathrm{~m}, 3 \mathrm{H}), 5.01(\mathrm{t}, J=7.2 \mathrm{~Hz}, 1 \mathrm{H}), 3.94(\mathrm{dd}, J=11.4,4.4 \mathrm{~Hz}, 2 \mathrm{H}), 3.67(\mathrm{~s}$, $3 \mathrm{H}), 3.39(\mathrm{t}, J=11.9 \mathrm{~Hz}, 2 \mathrm{H}), 3.30(\mathrm{~s}, 2 \mathrm{H}), 2.70(\mathrm{~m}, 1 \mathrm{H}), 2.32(\mathrm{t}, J=7.6 \mathrm{~Hz}, 2 \mathrm{H}), 2.10(\mathrm{q}, J=7.6 \mathrm{~Hz}$, $2 \mathrm{H}), 1.64(\mathrm{~m}, 4 \mathrm{H}), 1.38$ (pent, $J=7.6 \mathrm{~Hz}, 2 \mathrm{H}), 1.30(\mathrm{~m}, 2 \mathrm{H})$; (The stereochemistry was confirmed by NOESY1D NMR spectroscopy); ${ }^{13} \mathrm{C} \mathrm{NMR}\left(\mathrm{CDCl}_{3}, 126 \mathrm{MHz}\right): \delta 174.3,141.7,140.9,129.3,128.4$, $128.1,126.1,68.6,51.7,40.1,37.8,34.2,31.4,29.8,27.3,24.9$; FTIR (thin film): 2946, 1736, 1435, $1127,1013,736,699 \mathrm{~cm}^{-1}$; HRMS $m / z$ calculated for $\mathrm{C}_{20} \mathrm{H}_{29} \mathrm{O}_{3}[\mathrm{M}+\mathrm{H}]^{+}:$317.2117, found 317.2114.

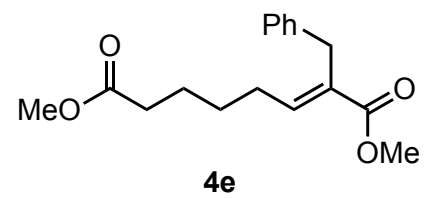

dimethyl $(\boldsymbol{E})$-2-benzyloct-2-enedioate $(\boldsymbol{E})-4 \mathrm{e})$. The general procedure was followed using $80 \mathrm{mg}$ of $\mathrm{KF} \cdot 2 \mathrm{H}_{2} \mathrm{O}(0.85 \mathrm{mmol}), 61 \mathrm{mg}$ of phenylboronic acid $3 \mathbf{a}(0.5 \mathrm{mmol}), 16 \mathrm{mg}$ of $\mathrm{Pd}_{2} \mathrm{dba}_{3} \cdot \mathrm{CHCl}_{3}(3.0$ $\mathrm{mol} \%), 8 \mathrm{~mL}$ of DMF, and a solution of $152 \mathrm{mg}$ of $2 \mathrm{e}(0.65 \mathrm{mmol})$ and $0.21 \mathrm{~mL}$ of methyl 5-hexenoate $1 \mathrm{a}(1.5 \mathrm{mmol})$ in $2.0 \mathrm{~mL}$ of DMF. Purification by silica gel flash chromatography (19:1 hexanes:ethyl acetate) led to the isolation of $4 \mathbf{e}$ and $\mathbf{5 e}$ as a colorless oil $(120 \mathrm{mg}, 83 \%$ as a 3.0:0.78:1.0 mixture of $(E)-4 \mathbf{e}:(Z)-4 \mathbf{e}: 5 \mathbf{e}$ isomers respectively), isomeric ratios were determined by $\mathrm{GC}_{\mathrm{R}}=0.40$ (3:1 hexanes:ethyl acetate, visualized by $254 \mathrm{~nm}$ light and PMA stain). ${ }^{1} \mathrm{H} \mathrm{NMR}\left(\mathrm{CDCl}_{3}, 500 \mathrm{MHz}\right): \delta 7.25(\mathrm{~m}$, 2H), $7.17(\mathrm{~m}, 3 \mathrm{H}), 6.92(\mathrm{t}, J=7.5 \mathrm{~Hz}, 1 \mathrm{H}), 3.69(\mathrm{~m}, 5 \mathrm{H}), 3.66(\mathrm{~s}, 3 \mathrm{H}), 2.29(\mathrm{~m}, 4 \mathrm{H}), 1.65$ (pent, $J=7.5$ $\mathrm{Hz}, 2 \mathrm{H}$ ), 1.49 (pent, $J=7.5 \mathrm{~Hz}, 2 \mathrm{H}$ ); (The stereochemistry was confirmed by NOESY1D NMR spectroscopy); ${ }^{13} \mathrm{C} \mathrm{NMR}\left(\mathrm{CDCl}_{3}, 126 \mathrm{MHz}\right): \delta 174.1,168.3,143.8,139.8,131.4,128.6,128.4,126.2,52.0$, 51.8, 34.0, 32.6, 28.8, 28.4, 24.9; FTIR (thin film): 2949, 1736, 1601, 1435, 1196, 1062, 736, $698 \mathrm{~cm}^{-1}$; HRMS $m / z$ calculated for $\mathrm{C}_{17} \mathrm{H}_{22} \mathrm{O}_{4} \mathrm{Na}[\mathrm{M}+\mathrm{Na}]^{+}: 313.1416$, found 313.1414 .

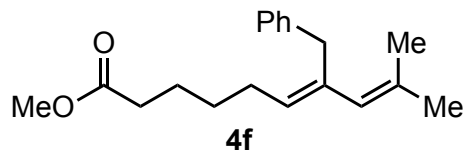


methyl $(\boldsymbol{E})$-7-benzyl-9-methyldeca-6,8-dienoate $(\boldsymbol{E})-4 f)$. The general procedure was followed using $80 \mathrm{mg}$ of $\mathrm{KF} \bullet 2 \mathrm{H}_{2} \mathrm{O}(0.85 \mathrm{mmol}), 61 \mathrm{mg}$ of phenylboronic acid $3 \mathbf{a}(0.5 \mathrm{mmol}), 16 \mathrm{mg}$ of $\mathrm{Pd}_{2} \mathrm{dba}_{3} \cdot \mathrm{CHCl}_{3}$ (3.0 mol\%), $8 \mathrm{~mL}$ of DMF, and a solution of $150 \mathrm{mg}$ of $\mathbf{2 f}(0.65 \mathrm{mmol})$ and $0.21 \mathrm{~mL}$ of methyl 5hexenoate 1a $(1.5 \mathrm{mmol})$ in $2.0 \mathrm{~mL}$ of DMF. Purification by silica gel flash chromatography $(100 \%$ hexanes $\rightarrow$ 97:3 hexanes:ethyl acetate) led to the isolation of $\mathbf{4 f}$ and $\mathbf{5 f}$ as a colorless oil (32 $\mathrm{mg}, 22 \%$ as a 10.4:2.5:1.0 mixture of $(E)-\mathbf{4 f}:(Z)-\mathbf{4 f : 5 f}$ isomers respectively), isomeric ratios were determined by GC, $\mathrm{R}_{f}=0.30$ (19:1 hexanes:ethyl acetate, visualized by $254 \mathrm{~nm}$ light and PMA stain). ${ }^{1} \mathrm{H} \mathrm{NMR}\left(\mathrm{CDCl}_{3}, 400\right.$ MHz): $\delta 7.28(\mathrm{~m}, 2 \mathrm{H}), 7.19(\mathrm{~m}, 3 \mathrm{H}), 5.74(\mathrm{~s}, 1 \mathrm{H}), 5.26(\mathrm{t}, J=7.2 \mathrm{~Hz}, 1 \mathrm{H}), 3.66(\mathrm{~s}, 3 \mathrm{H}), 3.32(\mathrm{~s}, 2 \mathrm{H})$, $2.32(\mathrm{t}, J=7.2 \mathrm{~Hz}, 2 \mathrm{H}), 2.09$ (q, $J=7.2 \mathrm{~Hz}, 2 \mathrm{H}), 1.72(\mathrm{~s}, 3 \mathrm{H}), 1.70-1.60(\mathrm{~m}, 5 \mathrm{H}), 1.41$ (pent, $J=7.6$ $\mathrm{Hz}, 2 \mathrm{H})$; (The stereochemistry was confirmed by NOESY1D NMR spectroscopy); ${ }^{13} \mathrm{C}$ NMR $\left(\mathrm{CDCl}_{3}\right.$, $100 \mathrm{MHz}): \delta 174.4,140.5,134.7,133.3,130.9,129.3,129.1,128.4,126.2,51.7,47.3,34.2,29.4,28.0$, 24.9, 17.8, 17.2; FTIR (thin film): 2931, 1739, 1653, 1600, 1436, 1171, 1029, 744, $670 \mathrm{~cm}^{-1}$; HRMS $m / z$ calculated for $\mathrm{C}_{19} \mathrm{H}_{26} \mathrm{O}_{2} \mathrm{Na}[\mathrm{M}+\mathrm{Na}]^{+}: 309.1855$, found 309.1828 .

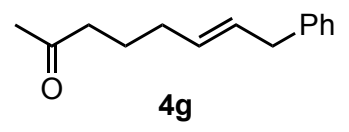

$(\boldsymbol{E})$-8-phenyloct-6-en-2-one $((\boldsymbol{E})-4 \mathrm{~g})$. The general procedure was followed using $80 \mathrm{mg}$ of $\mathrm{KF} \cdot 2 \mathrm{H}_{2} \mathrm{O}$ $(0.85 \mathrm{mmol}), 61 \mathrm{mg}$ of phenylboronic acid $3 \mathbf{a}(0.5 \mathrm{mmol}), 16 \mathrm{mg}$ of $\mathrm{Pd}_{2} \mathrm{dba}_{3} \cdot \mathrm{CHCl}_{3}(3.0 \mathrm{~mol} \%), 8 \mathrm{~mL}$ of DMF, and a solution of $212 \mathrm{mg}$ of $\mathbf{2 b}(0.65 \mathrm{mmol})$ and $0.18 \mathrm{~mL}$ of hex-5-en-2-one $\mathbf{1 b}(1.5 \mathrm{mmol})$ in $2.0 \mathrm{~mL}$ of DMF. Purification by silica gel flash chromatography (95:5 hexanes: ethyl acetate) led to the isolation of $\mathbf{4 g}$ and $\mathbf{5 g}$ as a colorless oil $(89 \mathrm{mg}, 88 \%$ as a 7.5:0.74:1.0 mixture of $(E)-\mathbf{4 g : ( Z ) - 4 g : 5 g}$ isomers respectively), isomeric ratios were determined by $\mathrm{GC}, \mathrm{R}_{f}=0.38$ (4:1 hexanes:ethyl acetate, visualized by $254 \mathrm{~nm}$ light and PMA stain). ${ }^{1} \mathrm{H}$ NMR $\left(\mathrm{CDCl}_{3}, 500 \mathrm{MHz}\right): \delta 7.30-7.27(\mathrm{~m}, 2 \mathrm{H}), 7.22-7.14$ $(\mathrm{m}, 3 \mathrm{H}), 5.66-5.54$ (app. dt, $J=15.2,7.6 \mathrm{~Hz}, 1 \mathrm{H}), 5.51-5.40$ (app. dt, $J=15.2,7.6 \mathrm{~Hz}, 1 \mathrm{H}), 3.33$ (d, $J=6.5 \mathrm{~Hz}, 2 \mathrm{H}), 2.42(\mathrm{t}, J=7.5 \mathrm{~Hz}, 2 \mathrm{H}), 2.12(\mathrm{~s}, 3 \mathrm{H}), 2.04(\mathrm{q}, J=7.2 \mathrm{~Hz}, 2 \mathrm{H}), 1.66$ (pent, $J=7.4 \mathrm{~Hz}$, $2 \mathrm{H}) ;{ }^{13} \mathrm{C} \mathrm{NMR}\left(\mathrm{CDCl}_{3}, 126 \mathrm{MHz}\right): \delta 209.0,140.8,130.8,129.9,128.44,128.35,125.9,43.0,39.0,31.8$, 29.9, 23.4; FTIR (thin film): 2938, 1715, 1451, $1373 \mathrm{~cm}^{-1}$; HRMS m/z calculated for $\mathrm{C}_{14} \mathrm{H}_{18} \mathrm{ONa}$ $[\mathrm{M}+\mathrm{Na}]^{+}: 225.1255$, found 225.1252 .

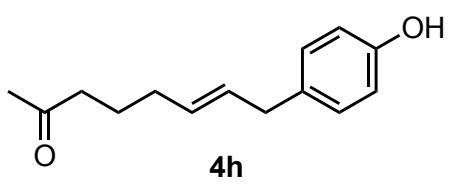

(E)-8-(4-hydroxyphenyl)oct-6-en-2-one $((\boldsymbol{E})-4 h)$. The general procedure was followed using $80 \mathrm{mg}$ of $\mathrm{KF} \cdot 2 \mathrm{H}_{2} \mathrm{O}(0.85 \mathrm{mmol}), 69 \mathrm{mg}$ of (4-hydroxyphenyl)boronic acid $3 \mathbf{b}(0.5 \mathrm{mmol}), 16 \mathrm{mg}$ of $\mathrm{Pd}_{2} \mathrm{dba}_{3} \cdot \mathrm{CHCl}_{3}(3.0 \mathrm{~mol} \%), 8 \mathrm{~mL}$ of DMF, and a solution of $212 \mathrm{mg}$ of $\mathbf{2 b}(0.65 \mathrm{mmol})$ and $0.18 \mathrm{~mL}$ of hex-5-en-2-one $\mathbf{1 b}(1.5 \mathrm{mmol})$ in $2.0 \mathrm{~mL}$ of DMF. Purification by silica gel flash chromatography (1:2 hexanes: ethyl acetate) led to the isolation of $\mathbf{4 h}$ and $\mathbf{5 h}$ as a colorless oil $(81 \mathrm{mg}, 74 \%$ as a 6.7:0.28:1.0 mixture of $(E)-\mathbf{4 h}:(Z)-\mathbf{4 h}: 5 \mathbf{h}$ isomers respectively), isomeric ratios were determined by GC, $\mathrm{R}_{f}=0.35$ (1:1 hexanes:ethyl acetate, visualized by $254 \mathrm{~nm}$ light and PMA stain). ${ }^{1} \mathrm{H} \mathrm{NMR}\left(\mathrm{CDCl}_{3}, 500\right.$ MHz): $\delta 7.02(\mathrm{~d}, J=8.5 \mathrm{~Hz}, 2 \mathrm{H}), 6.77(\mathrm{~d}, J=8.5 \mathrm{~Hz}, 2 \mathrm{H}), 5.64-5.48$ (app. dt, $J=15.2,5.4 \mathrm{~Hz}, 1 \mathrm{H})$, $5.45-5.39$ (app. dt, $J=15.1,6.7 \mathrm{~Hz}, 1 \mathrm{H}), 5.31(\mathrm{~s}, 1 \mathrm{H}), 3.24(\mathrm{~d}, J=7.0 \mathrm{~Hz}, 2 \mathrm{H}), 2.43(\mathrm{t}, J=7.5 \mathrm{~Hz}$, 2H), 2.13 (s, 3H), 2.04 (q, $J=7.0 \mathrm{~Hz}, 2 \mathrm{H}), 1.66$ (pent, $J=7.4 \mathrm{~Hz}, 2 \mathrm{H}) ;{ }^{13} \mathrm{C} \mathrm{NMR}\left(\mathrm{CDCl}_{3}, 126 \mathrm{MHz}\right): \delta$ 209.8, 153.9, 132.8, 130.4, 129.5, 115.2, 43.0, 38.1, 31.8, 29.9, 23.4; FTIR (thin film): 3369, 2933, 2359, 2338, 1699, $1514 \mathrm{~cm}^{-1}$; HRMS $\mathrm{m} / \mathrm{z}$ calculated for $\mathrm{C}_{14} \mathrm{H}_{18} \mathrm{O}_{2} \mathrm{Na}[\mathrm{M}+\mathrm{Na}]^{+}: 241.1204$, found 241.1201.

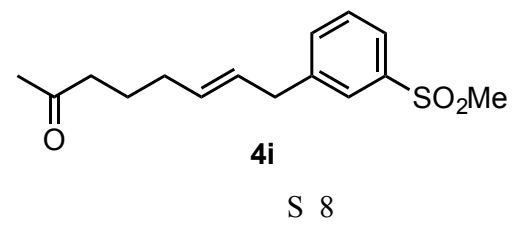


(E)-8-(3-(methylsulfonyl)phenyl)oct-6-en-2-one ((E)-4i). The general procedure was followed using $80 \mathrm{mg}$ of $\mathrm{KF} \cdot 2 \mathrm{H}_{2} \mathrm{O}(0.85 \mathrm{mmol}), 100 \mathrm{mg}$ of (3-(methylsulfonyl)phenyl)boronic acid 3c $(0.5 \mathrm{mmol}), 16$ $\mathrm{mg}$ of $\mathrm{Pd}_{2} \mathrm{dba}_{3} \cdot \mathrm{CHCl}_{3}(3.0 \mathrm{~mol} \%), 8 \mathrm{~mL}$ of DMF, and a solution of $212 \mathrm{mg}$ of $\mathbf{2 b}(0.65 \mathrm{mmol})$ and 0.18 $\mathrm{mL}$ of hex-5-en-2-one $\mathbf{1 b}(1.5 \mathrm{mmol})$ in $2.0 \mathrm{~mL}$ of DMF. Purification by silica gel flash chromatography (10:1 hexanes: ethyl acetate) led to the isolation of $\mathbf{4 i}$ and $\mathbf{5 i}$ as a colorless oil (121 $\mathrm{mg}, 86 \%$ as a 16.3:3.0:1.0 mixture of $(E)-4 \mathbf{i}:(Z)-4 \mathbf{i}: 5 \mathbf{i}$ isomers respectively), isomeric ratios were determined by GC, $\mathrm{R}_{f}=0.36$ (4:1 hexanes:ethyl acetate, visualized by $254 \mathrm{~nm}$ light and PMA stain). ${ }^{1} \mathrm{H} \mathrm{NMR}\left(\mathrm{CDCl}_{3}, 500\right.$ $\mathrm{MHz}): \delta 7.82-7.73(\mathrm{~m}, 2 \mathrm{H}), 7.54-7.40(\mathrm{~m}, 2 \mathrm{H}), 5.56(\mathrm{app} . \mathrm{dt}, J=15.3,6.1 \mathrm{~Hz}, 1 \mathrm{H}), 5.50(\mathrm{app} . \mathrm{dt}, J=$ $15.3,6.3 \mathrm{~Hz}, 1 \mathrm{H}), 3.41(\mathrm{~d}, J=6.1 \mathrm{~Hz}, 2 \mathrm{H}), 3.05(\mathrm{~s}, 3 \mathrm{H}), 2.43(\mathrm{t}, J=7.4 \mathrm{~Hz}, 2 \mathrm{H}), 2.13(\mathrm{~s}, 3 \mathrm{H}), 2.05(\mathrm{q}, J$ $=7.0 \mathrm{~Hz}, 2 \mathrm{H}), 1.67$ (pent, $J=7.4 \mathrm{~Hz}, 2 \mathrm{H}) ;{ }^{13} \mathrm{C} \mathrm{NMR}\left(\mathrm{CDCl}_{3}, 126 \mathrm{MHz}\right): \delta 208.2,142.7,140.6,133.9$, 132.4, 129.4, 128.3, 127.1, 125.0, 44.5, 42.9, 38.7, 31.8, 30.0, 23.2; FTIR (thin film): 2928, 1171, 1299, $1144 \mathrm{~cm}^{-1}$; HRMS $\mathrm{m} / z$ calculated for $\mathrm{C}_{15} \mathrm{H}_{20} \mathrm{O}_{3} \mathrm{SNa}[\mathrm{M}+\mathrm{Na}]^{+}: 303.1031$, found 303.1031 .

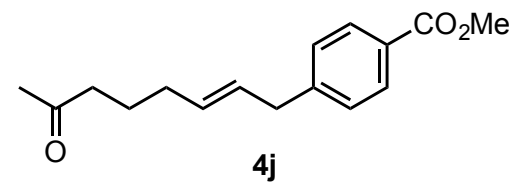

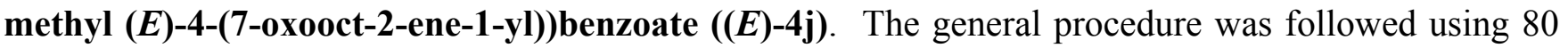
$\mathrm{mg}$ of $\mathrm{KF} \cdot 2 \mathrm{H}_{2} \mathrm{O} \quad(0.85 \mathrm{mmol}), 90 \mathrm{mg}$ of (4-(methoxycarbonyl)phenyl)boronic acid $3 \mathbf{d}(0.5 \mathrm{mmol}), 16$ $\mathrm{mg}$ of $\mathrm{Pd}_{2} \mathrm{dba}_{3} \cdot \mathrm{CHCl}_{3}(3.0 \mathrm{~mol} \%), 8 \mathrm{~mL}$ of DMF, and a solution of $212 \mathrm{mg}$ of $\mathbf{2 b}(0.65 \mathrm{mmol})$ and 0.18 $\mathrm{mL}$ of hex-5-en-2-one $\mathbf{1 b}(1.5 \mathrm{mmol})$ in $2.0 \mathrm{~mL}$ of DMF. Purification by silica gel flash chromatography (4:1 hexanes:ethyl acetate) led to the isolation of $\mathbf{4 j}$ and $\mathbf{5 j}$ as a colorless oil (92 $\mathrm{mg}, 71 \%$ as a 14.1:1.9:1.0 mixture of $(E) \mathbf{- 4 j}:(Z) \mathbf{- 4 j}: \mathbf{5 j}$ isomers respectively), isomeric ratios were determined by GC, $\mathrm{R}_{f}=0.38$ (1:1 hexanes:ethyl acetate, visualized by $254 \mathrm{~nm}$ light and PMA stain). ${ }^{1} \mathrm{H} \mathrm{NMR}\left(\mathrm{CDCl}_{3}, 500\right.$ MHz): $\delta 7.95(\mathrm{~d}, J=8.3 \mathrm{~Hz}, 2 \mathrm{H}), 7.23(\mathrm{~d}, J=8.5 \mathrm{~Hz}, 2 \mathrm{H}), 5.56($ app. dt, $J=15.2,6.6 \mathrm{~Hz}, 1 \mathrm{H}), 5.47$ (app. dt, $J=15.2,6.6 \mathrm{~Hz}, 1 \mathrm{H}), 3.89$ (s, 3H), $3.37(\mathrm{~d}, J=6.5 \mathrm{~Hz}, 2 \mathrm{H}), 2.41$ (t, $J=7.4 \mathrm{~Hz}, 2 \mathrm{H}), 2.11$ (s, $3 \mathrm{H}), 2.04(\mathrm{q}, J=6.6 \mathrm{~Hz}, 2 \mathrm{H}), 1.66$ (pent, $J=7.4 \mathrm{~Hz}, 2 \mathrm{H}) ;{ }^{13} \mathrm{C} \mathrm{NMR}\left(\mathrm{CDCl}_{3}, 126 \mathrm{MHz}\right): \delta 208.8,167.1$, 146.0, 131.7, 129.7, 128.8, 128.5, 128.0, 51.9, 42.9, 39.0, 31.8, 29.9, 23.3; FTIR (thin film): 2925, 2854, $1708,1397 \mathrm{~cm}^{-1}$; HRMS $\mathrm{m} / z$ calculated for $\mathrm{C}_{16} \mathrm{H}_{20} \mathrm{O}_{3} \mathrm{Na}[\mathrm{M}+\mathrm{Na}]^{+}: 283.1310$, found 283.1308 .

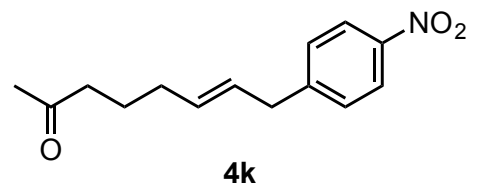

$(\boldsymbol{E})$-8-(4-nitrophenyl)oct-6-en-2-one $((\boldsymbol{E})-4 \mathbf{k})$. The general procedure was followed using $80 \mathrm{mg}$ of $\mathrm{KF} \cdot 2 \mathrm{H}_{2} \mathrm{O}(0.85 \mathrm{mmol}), 83 \mathrm{mg}$ of (4-nitrophenyl)boronic acid $3 \mathbf{e}(0.5 \mathrm{mmol}), 16 \mathrm{mg}$ of $\mathrm{Pd}_{2} \mathrm{dba}_{3} \bullet \mathrm{CHCl}_{3}$ (3.0 $\mathrm{mol} \%), 8 \mathrm{~mL}$ of DMF, and a solution of $212 \mathrm{mg}$ of $\mathbf{2 b}(0.65 \mathrm{mmol})$ and $0.18 \mathrm{~mL}$ of hex-5-en-2one $1 \mathbf{b}(1.5 \mathrm{mmol})$ in $2.0 \mathrm{~mL}$ of DMF. Purification by silica gel flash chromatography (19:1 hexanes: ethyl acetate) led to the isolation of $\mathbf{4 k}$ and $\mathbf{5 k}$ as a colorless oil (49 $\mathrm{mg}, 40 \%$ as a 16:2.85:1.0 mixture of $(E)-4 \mathbf{k}:(Z)-4 \mathbf{k}: 5 \mathbf{k}$ isomers respectively), isomeric ratios were determined by $\mathrm{GC}, \mathrm{R}_{f}=0.45$ (4:1 hexanes:ethyl acetate, visualized by $254 \mathrm{~nm}$ light and PMA stain). ${ }^{1} \mathrm{H}$ NMR ( $\left.\mathrm{CDCl}_{3}, 500 \mathrm{MHz}\right): \delta 8.14(\mathrm{~d}, J$ $=8.7 \mathrm{~Hz}, 2 \mathrm{H}), 7.32(\mathrm{~d}, J=8.8 \mathrm{~Hz}, 2 \mathrm{H}), 5.56($ app. dt, $J=15.2,6.2 \mathrm{~Hz}, 1 \mathrm{H}), 5.51$ (app. dt, $J=15.3,6.1$ $\mathrm{Hz}, 1 \mathrm{H}), 3.42$ (d, $J=5.8 \mathrm{~Hz}, 2 \mathrm{H}), 2.43$ (t, $J=7.4 \mathrm{~Hz}, 2 \mathrm{H}), 2.12(\mathrm{~s}, 3 \mathrm{H}), 2.05$ (q, $J=6.8 \mathrm{~Hz}, 2 \mathrm{H}), 1.67$ (pent, $J=7.4 \mathrm{~Hz}, 2 \mathrm{H}) ;{ }^{13} \mathrm{C} \mathrm{NMR}\left(\mathrm{CDCl}_{3}, 126 \mathrm{MHz}\right): \delta 209.5,149.4,147.2,133.4,130.0,128.6,124.4$, 43.7, 39.6, 32.6, 30.7, 24.0; FTIR (thin film): 2935, 1714, 1597, 1517, $1345 \mathrm{~cm}^{-1}$; HRMS m/z calculated for $\mathrm{C}_{14} \mathrm{H}_{17} \mathrm{NO}_{3} \mathrm{Na}[\mathrm{M}+\mathrm{Na}]^{+}: 270.1106$, found 270.1101 .

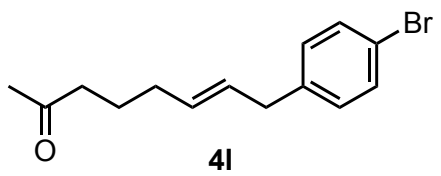


(E)-8-(4-bromophenyl)oct-6-en-2-one $((\boldsymbol{E})-4 \mathrm{l})$. The general procedure was followed using $80 \mathrm{mg}$ of $\mathrm{KF} \cdot 2 \mathrm{H}_{2} \mathrm{O} \quad(0.85 \mathrm{mmol}), 100 \mathrm{mg}$ of (4-bromophenyl)boronic acid $\mathbf{3 f}(0.5 \mathrm{mmol}), 16 \mathrm{mg}$ of $\mathrm{Pd}_{2} \mathrm{dba}_{3} \cdot \mathrm{CHCl}_{3}(3.0 \mathrm{~mol} \%), 8 \mathrm{~mL}$ of DMF, and a solution of $212 \mathrm{mg}$ of $\mathbf{2 b}(0.65 \mathrm{mmol})$ and $0.18 \mathrm{~mL}$ of hex-5-en-2-one $\mathbf{1 b}(1.5 \mathrm{mmol})$ in $2.0 \mathrm{~mL}$ of DMF. Purification by silica gel flash chromatography (20:1 hexanes:ethyl acetate) led to the isolation of $\mathbf{4 l}$ and $\mathbf{5 l}$ as a colorless oil (48 $\mathbf{m g}, 34 \%$ as a 15.7:2.3:1.0 mixture of $(E)-41:(Z)-41: 51$ isomers respectively), isomeric ratios were determined by GC, $\mathrm{R}_{f}=0.42$ (4:1 hexanes:ethyl acetate, visualized by $254 \mathrm{~nm}$ light and PMA stain). ${ }^{1} \mathrm{H} \mathrm{NMR}\left(\mathrm{CDCl}_{3}, 500\right.$ MHz): $\delta 7.40(\mathrm{~d}, J=8.3 \mathrm{~Hz}, 2 \mathrm{H}), 7.04(\mathrm{~d}, J=8.2 \mathrm{~Hz}, 2 \mathrm{H}), 5.54$ (app. dt, $J=15.2,6.6 \mathrm{~Hz}, 1 \mathrm{H}), 5.45$ (app. dt, $J=15.2,6.6 \mathrm{~Hz}, 1 \mathrm{H}), 3.27(\mathrm{~d}, J=6.5 \mathrm{~Hz}, 2 \mathrm{H}), 2.41(\mathrm{t}, J=7.4 \mathrm{~Hz}, 2 \mathrm{H}), 2.12(\mathrm{~s}, 3 \mathrm{H}), 2.03(\mathrm{q}, J$ $=7.0 \mathrm{~Hz}, 2 \mathrm{H}), 1.66$ (pent, $J=7.5 \mathrm{~Hz}, 2 \mathrm{H}) ;{ }^{13} \mathrm{C} \mathrm{NMR}\left(\mathrm{CDCl}_{3}, 126 \mathrm{MHz}\right): \delta 209.0,139.8,131.38$, 131.36, 130.2, 129.2, 119.7, 43.0, 38.4, 31.8, 30.0, 23.4; FTIR (thin film): 2924, 2852, 2362, 2342, $1715,1488,1071 \mathrm{~cm}^{-1}$; HRMS $m / z$ calculated for $\mathrm{C}_{14} \mathrm{H}_{17} \mathrm{O}_{3} \mathrm{NaBr}[\mathrm{M}+\mathrm{Na}]^{+}: 303.0360$, found 303.0368.

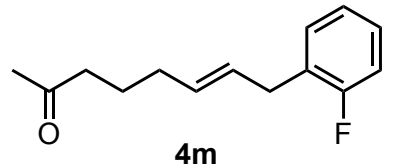

$(\boldsymbol{E})$-8-(2-fluorophenyl)oct-6-en-2-one $((\boldsymbol{E})-\mathbf{4 m})$. The general procedure was followed using $80 \mathrm{mg}$ of $\mathrm{KF} \cdot 2 \mathrm{H}_{2} \mathrm{O} \quad(0.85 \mathrm{mmol}), 70 \mathrm{mg}$ of (2-fluorophenyl)boronic acid $\mathbf{3 g}(0.5 \mathrm{mmol}), 16 \mathrm{mg}$ of $\mathrm{Pd}_{2} \mathrm{dba}_{3} \cdot \mathrm{CHCl}_{3}(3.0 \mathrm{~mol} \%), 8 \mathrm{~mL}$ of DMF, and a solution of $212 \mathrm{mg}$ of $\mathbf{2 b}(0.65 \mathrm{mmol})$ and $0.18 \mathrm{~mL}$ of hex-5-en-2-one $\mathbf{1 b}(1.5 \mathrm{mmol})$ in $2.0 \mathrm{~mL}$ of DMF. Purification by silica gel flash chromatography (10:1 hexanes:ethyl acetate) led to the isolation of $\mathbf{4 m}$ and $\mathbf{5 m}$ as a colorless oil (40 $\mathbf{m g}, 36 \%$ as a 14.8:1.2:1.0 mixture of $(E)-\mathbf{4 m :}(Z)-\mathbf{4 m}: \mathbf{5 m}$ isomers respectively), isomeric ratios were determined by $\mathrm{GC}, \mathrm{R}_{f}=0.42$ (4:1 hexanes: ethyl acetate, visualized by $254 \mathrm{~nm}$ light and PMA stain). ${ }^{1} \mathrm{H} \mathrm{NMR}\left(\mathrm{CDCl}_{3}\right.$, $500 \mathrm{MHz}): \delta 7.16(\mathrm{t}, J=7.2 \mathrm{~Hz}, 2 \mathrm{H}), 7.06(\mathrm{t}, J=6.8 \mathrm{~Hz}, 1 \mathrm{H}), 5.56$ (app. dt, $J=15.2,6.5 \mathrm{~Hz}, 1 \mathrm{H}), 5.45$ (app. dt, $J=15.3,6.7 \mathrm{~Hz}, 1 \mathrm{H}), 3.34(\mathrm{~d}, J=6.2 \mathrm{~Hz}, 2 \mathrm{H}), 2.41$ (t, $J=7.4 \mathrm{~Hz}, 2 \mathrm{H}), 2.11$ (s, 3H), $2.03(\mathrm{q}, J$ $=6.7 \mathrm{~Hz}, 2 \mathrm{H}), 1.65$ (pent, $J=7.4 \mathrm{~Hz}, 2 \mathrm{H}) ;{ }^{13} \mathrm{C} \mathrm{NMR}\left(\mathrm{CDCl}_{3}, 126 \mathrm{MHz}\right): \delta 209.0,160.9(\mathrm{~d}, J=245.2$ $\mathrm{Hz}), 131.2,130.5$ (d, $J=4.9 \mathrm{~Hz}), 128.2,127.66(\mathrm{~d}, J=15.8 \mathrm{~Hz}), 127.65(\mathrm{~d}, J=8.0 \mathrm{~Hz}), 123.9(\mathrm{~d}, J=$ $3.6 \mathrm{~Hz}), 115.2(\mathrm{~d}, J=22.0 \mathrm{~Hz}), 42.9,31.9,31.7,29.9,23.3$; FTIR (thin film): 2918, 2849, 1716, 1491, $1229 \mathrm{~cm}^{-1}$; HRMS m/z calculated for $\mathrm{C}_{14} \mathrm{H}_{17} \mathrm{ONaF}[\mathrm{M}+\mathrm{Na}]^{+}: 243.1161$, found 243.1159 .

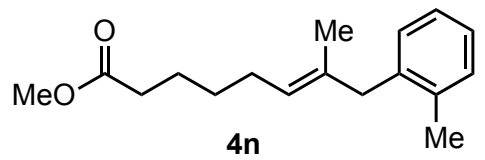

methyl $(E)$-7-methyl-8-(o-tolyl)oct-6-enoate $((\boldsymbol{E})-4 n)$. The general procedure was followed using 80 $\mathrm{mg}$ of $\mathrm{KF} \bullet 2 \mathrm{H}_{2} \mathrm{O}(0.85 \mathrm{mmol}), 68 \mathrm{mg}$ of $o$-tolylboronic acid $\mathbf{3 h}(0.5 \mathrm{mmol}), 16 \mathrm{mg}$ of $\mathrm{Pd}_{2} \mathrm{dba}_{3} \bullet \mathrm{CHCl}_{3}$ $(3.0 \mathrm{~mol} \%), 8 \mathrm{~mL}$ of DMF, and a solution of $221 \mathrm{mg}$ of $\mathbf{2 a}(0.65 \mathrm{mmol})$ and $0.21 \mathrm{~mL}$ of methyl 5hexenoate 1a $(1.5 \mathrm{mmol})$ in $2.0 \mathrm{~mL}$ of DMF. Purification by silica gel flash chromatography (19:1 hexanes:ethyl acetate) led to the isolation of $\mathbf{4 n}$ and $\mathbf{5 n}$ as a colorless oil (92 $\mathrm{mg}, 71 \%$ as a 3.9:0.75:1.0 mixture of $(E)-\mathbf{4 n}:(Z)-\mathbf{4 n}: \mathbf{5 n}$ isomers respectively), isomeric ratios were determined by $\mathrm{GC}, \mathrm{R}_{f}=0.26(19: 1$ hexanes:ethyl acetate, visualized by $254 \mathrm{~nm}$ light and PMA stain). ${ }^{1} \mathrm{H} \mathrm{NMR}\left(\mathrm{CDCl}_{3}, 400 \mathrm{MHz}\right): \delta 7.11$ $(\mathrm{m}, 4 \mathrm{H}), 5.03(\mathrm{t}, J=7.4 \mathrm{~Hz}, 1 \mathrm{H}), 3.66(\mathrm{~s}, 3 \mathrm{H}), 3.27(\mathrm{~s}, 2 \mathrm{H}), 2.30(\mathrm{t}, J=7.2 \mathrm{~Hz}, 2 \mathrm{H}), 2.26(\mathrm{~s}, 3 \mathrm{H}), 2.03$ $(\mathrm{q}, J=7.2 \mathrm{~Hz}, 2 \mathrm{H}), 1.66-1.56(\mathrm{~m}, 5 \mathrm{H}), 1.35$ (pent, $J=7.6 \mathrm{~Hz}, 2 \mathrm{H})$; (The stereochemistry was confirmed by NOESY1D NMR spectroscopy); The following signals can be assigned to $(Z)-4 \mathbf{n}:{ }^{1} \mathrm{H} \mathrm{NMR}\left(\mathrm{CDCl}_{3}\right.$, $400 \mathrm{MHz}): \delta 3.33(\mathrm{~s}, 2 \mathrm{H}) ;{ }^{13} \mathrm{C} \mathrm{NMR}\left(\mathrm{CDCl}_{3}, 126 \mathrm{MHz}\right): \delta 174.5,138.4,137.1,133.9,130.3,129.9$, 126.3, 126.0, 125.9, 51.7, 43.4, 34.2, 29.4, 27.8, 24.8, 19.7, 16.6; FTIR (thin film): 2930, 1603, 1435, $1169,1051,888,738 \mathrm{~cm}^{-1}$; HRMS $m / z$ calculated for $\mathrm{C}_{17} \mathrm{H}_{24} \mathrm{O}_{2} \mathrm{Na}[\mathrm{M}+\mathrm{Na}]^{+}: 283.1674$, found 283.1670. 


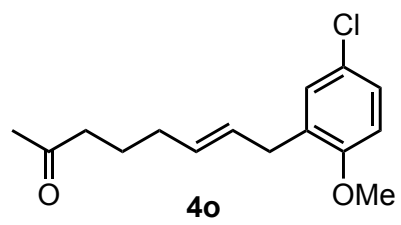

(E)-8-(5-chloro-2-methoxyphenyl)oct-6-en-2-one $((\boldsymbol{E})-40)$. The general procedure was followed using $80 \mathrm{mg}$ of $\mathrm{KF} \bullet 2 \mathrm{H}_{2} \mathrm{O}(0.85 \mathrm{mmol}), 93 \mathrm{mg}$ of (5-chloro-2-methoxyphenyl)boronic acid $\mathbf{3 i}(0.5 \mathrm{mmol})$, $16 \mathrm{mg}$ of $\mathrm{Pd}_{2} \mathrm{dba}_{3} \cdot \mathrm{CHCl}_{3}(3.0 \mathrm{~mol} \%), 8 \mathrm{~mL}$ of DMF, and a solution of $221 \mathrm{mg}$ of $\mathbf{2 b}(0.65 \mathrm{mmol})$ and $0.21 \mathrm{~mL}$ of methyl 5-hexenoate $\mathbf{1 b}(1.5 \mathrm{mmol})$ in $2.0 \mathrm{~mL}$ of DMF. Purification by silica gel flash chromatography (10:1 hexanes:ethyl acetate) led to the isolation of $\mathbf{4 0}$ and $\mathbf{5 0}$ as a colorless oil (73 $\mathrm{mg}$, $55 \%$ as a 17.5:1.5:1.0 mixture of $(E)-\mathbf{4 0}:(Z)-\mathbf{4 0 : 5 0}$ isomers respectively), isomeric ratios were determined by $\mathrm{GC}, \mathrm{R}_{f}=0.35$ (4:1 hexanes:ethyl acetate, visualized by $254 \mathrm{~nm}$ light and PMA stain). ${ }^{1} \mathrm{H}$ NMR $\left(\mathrm{CDCl}_{3}, 500 \mathrm{MHz}\right): \delta 7.12(\mathrm{dd}, J=8.8,2.8 \mathrm{~Hz}, 1 \mathrm{H}), 7.08(\mathrm{~d}, J=2.5 \mathrm{~Hz}, 1 \mathrm{H}), 6.75(\mathrm{~d}, J=8.7 \mathrm{~Hz}$, 1H), 5.54 (app. dt, $J=15.2,6.7 \mathrm{~Hz}, 1 \mathrm{H}), 5.43$ (app. dt, $J=15.2,6.7 \mathrm{~Hz}, 1 \mathrm{H}), 3.80$ (s, 3H), 3.27 (d, $J=$ $6.6 \mathrm{~Hz}, 2 \mathrm{H}), 2.42(\mathrm{t}, J=7.4 \mathrm{~Hz}, 2 \mathrm{H}), 2.12(\mathrm{~s}, 3 \mathrm{H}), 2.03(\mathrm{q}, J=7.0 \mathrm{~Hz}, 2 \mathrm{H}), 1.65$ (pent, $J=7.4 \mathrm{~Hz}, 2 \mathrm{H})$; ${ }^{13} \mathrm{C} \mathrm{NMR}\left(\mathrm{CDCl}_{3}, 126 \mathrm{MHz}\right): \delta 209.0,155.8,131.3,129.4,128.3,126.7,125.3,111.4,55.6,42.9,32.7$, 31.8, 29.9, 23.4; FTIR (thin film): 2937, 2838, 1715, 1488, 1246, 1126, $1030 \mathrm{~cm}^{-1}$; HRMS m/z calculated for $\mathrm{C}_{15} \mathrm{H}_{19} \mathrm{O}_{2} \mathrm{NaCl}[\mathrm{M}+\mathrm{Na}]^{+}:$289.0971, found 289.0966.

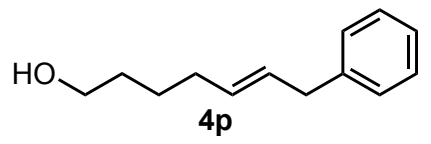

$(\boldsymbol{E})$-7-phenylhept-5-en-1-ol $((\boldsymbol{E})-4 \mathbf{p})$. The general procedure was followed using $80 \mathrm{mg}$ of $\mathrm{KF} \bullet 2 \mathrm{H}_{2} \mathrm{O}$ (0.85 mmol), $61 \mathrm{mg}$ of phenylboronic acid 3a $(0.5 \mathrm{mmol}), 16 \mathrm{mg}$ of $\mathrm{Pd}_{2} \mathrm{dba}_{3} \bullet \mathrm{CHCl}_{3}(3.0 \mathrm{~mol} \%), 8 \mathrm{~mL}$ of DMF, and a solution of $221 \mathrm{mg}$ of $\mathbf{2 b}(0.65 \mathrm{mmol})$ and $0.16 \mathrm{~mL}$ of pent-4-en-1-ol $1 \mathbf{c}(1.5 \mathrm{mmol})$ in $2.0 \mathrm{~mL}$ of DMF. Purification by silica gel flash chromatography (2:1 hexanes:ethyl acetate) led to the isolation of $\mathbf{4 p}$ and $\mathbf{5 p}$ as a colorless oil $(60 \mathrm{mg}, 59 \%$ as a 4.5:0.3:1.0 mixture of $(E)-\mathbf{4 p : ( Z ) - 4 p : 5 p ~ i s o - ~}$ mers respectively), isomeric ratios were determined by $\mathrm{GC}, \mathrm{R}_{f}=0.45$ (1:1 hexanes:ethyl acetate, visualized by $254 \mathrm{~nm}$ light and PMA stain). ${ }^{1} \mathrm{H}$ NMR $\left(\mathrm{CDCl}_{3}, 400 \mathrm{MHz}\right): \delta 7.32-7.24(\mathrm{~m}, 2 \mathrm{H}), 7.22-7.15$ $(\mathrm{m}, 3 \mathrm{H}), 5.59$ (app. dt, $J=15.0,6.6 \mathrm{~Hz}, 1 \mathrm{H}), 5.51$ (app. dt, $J=15.2,6.6 \mathrm{~Hz}, 1 \mathrm{H}), 3.64$ (td, $J=6.5,0.5$ $\mathrm{Hz}, 2 \mathrm{H}), 3.33(\mathrm{~d}, J=6.5 \mathrm{~Hz}, 2 \mathrm{H}), 2.07(\mathrm{q}, J=7.3 \mathrm{~Hz}, 2 \mathrm{H}), 1.64-1.54(\mathrm{~m}, 2 \mathrm{H}), 1.48-1.42(\mathrm{~m}, 2 \mathrm{H}) ;{ }^{13} \mathrm{C}$ NMR $\left(\mathrm{CDCl}_{3}, 126 \mathrm{MHz}\right): \delta 141.0,131.5,129.2,128.4,128.3,125.9,62.9,39.0,32.3,32.2,25.6$; FTIR (thin film): 3330, 2932, 2361, 2342, 1494, $1453 \mathrm{~cm}^{-1}$; HRMS $\mathrm{m} / z$ calculated for $\mathrm{C}_{13} \mathrm{H}_{18} \mathrm{O}_{2} \mathrm{Na}[\mathrm{M}+\mathrm{Na}]^{+}$: 213.1204, found 213.1201.

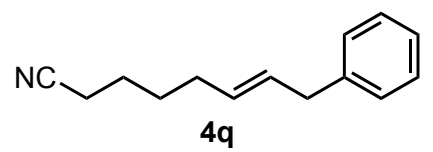

$(\boldsymbol{E})$-8-phenyloct-6-enenitrile $((\boldsymbol{E})-\mathbf{4 q})$. The general procedure was followed using $80 \mathrm{mg}$ of $\mathrm{KF} \cdot 2 \mathrm{H}_{2} \mathrm{O}$ $(0.85 \mathrm{mmol}), 61 \mathrm{mg}$ of phenylboronic acid 3a $(0.5 \mathrm{mmol}), 16 \mathrm{mg}$ of $\mathrm{Pd}_{2} \mathrm{dba}_{3} \bullet \mathrm{CHCl}_{3}(3.0 \mathrm{~mol} \%), 8 \mathrm{~mL}$ of DMF, and a solution of $221 \mathrm{mg}$ of $\mathbf{2 b}(0.65 \mathrm{mmol})$ and $0.16 \mathrm{~mL}$ of methyl 5-hexenoate $\mathbf{1 d}(1.5$ $\mathrm{mmol})$ in $2.0 \mathrm{~mL}$ of DMF. Purification by silica gel flash chromatography (20:1 hexanes:ethyl acetate) led to the isolation of $\mathbf{4 q}$ and $\mathbf{5 q}$ as a colorless oil $(82 \mathrm{mg}, 82 \%$ as a 10.5:0. 5:1.0 mixture of $(E)-\mathbf{4 q}:(Z)-$ 4q:5q isomers respectively), isomeric ratios were determined by $\mathrm{GC}, \mathrm{R}_{f}=0.35$ (4:1 hexanes:ethyl acetate, visualized by $254 \mathrm{~nm}$ light and PMA stain). ${ }^{1} \mathrm{H} \mathrm{NMR}\left(\mathrm{CDCl}_{3}, 500 \mathrm{MHz}\right): \delta 7.29(\mathrm{t}, J=7.6 \mathrm{~Hz}$, 2H), $7.21-7.17$ (m, 3H), 5.61 (app. dt, $J=15.2,7.5 \mathrm{~Hz}, 1 \mathrm{H}), 5.47$ (app. dt, $J=15.2,6.7 \mathrm{~Hz}, 1 \mathrm{H}$ ), 3.34 $(\mathrm{d}, J=6.7 \mathrm{~Hz}, 2 \mathrm{H}), 2.33(\mathrm{t}, J=7.1 \mathrm{~Hz}, 2 \mathrm{H}), 2.08(\mathrm{q}, J=7.1 \mathrm{~Hz}, 2 \mathrm{H}), 1.67$ (pent, $J=7.4 \mathrm{~Hz}, 2 \mathrm{H}), 1.54$ (pent, $J=7.5 \mathrm{~Hz}, 2 \mathrm{H}) ;{ }^{13} \mathrm{C} \mathrm{NMR}\left(\mathrm{CDCl}_{3}, 126 \mathrm{MHz}\right): \delta 140.7,130.4,130.0,128.42,128.37,126.0$, 119.7, 39.0, 31.5, 28.3, 24.8, 17.0; FTIR (thin film): 2935, 2869, 1722, 1495, $1452 \mathrm{~cm}^{-1}$; HRMS m/z calculated for $\mathrm{C}_{14} \mathrm{H}_{18} \mathrm{O}_{2} \mathrm{~N}[\mathrm{M}+\mathrm{H}]^{+}: 200.1439$, found 200.1442 . 


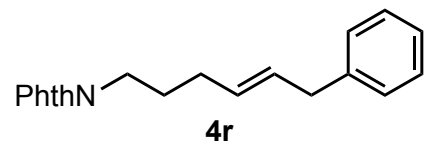

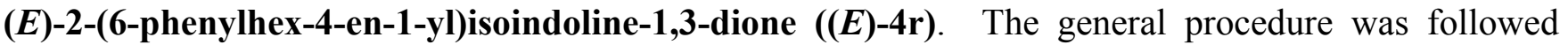
using $80 \mathrm{mg}$ of $\mathrm{KF} \cdot 2 \mathrm{H}_{2} \mathrm{O}(0.85 \mathrm{mmol}), 61 \mathrm{mg}$ of phenylboronic acid $\mathbf{3 a}(0.5 \mathrm{mmol}), 16 \mathrm{mg}$ of $\mathrm{Pd}_{2} \mathrm{dba}_{3} \cdot \mathrm{CHCl}_{3}(3.0 \mathrm{~mol} \%), 8 \mathrm{~mL}$ of DMF, and a solution of $221 \mathrm{mg}$ of $\mathbf{2 b}(0.65 \mathrm{mmol})$ and $151 \mathrm{mg}$ of 2-(but-3-en-1-yl)isoindoline-1,3-dione 1e (1.5 mmol) in $2.0 \mathrm{~mL}$ of DMF. Purification by silica gel flash chromatography (10:1 hexanes:ethyl acetate) led to the isolation of $\mathbf{4 r}$ and $\mathbf{5 r}$ as a colorless oil (136 $\mathrm{mg}$, $89 \%$ as a $9.5: 1.5: 1.0$ mixture of $(E)-4 \mathbf{r}:(Z)-4 \mathbf{r}: 5 \mathbf{r}$ isomers respectively), isomeric ratios were determined by $\mathrm{GC}, \mathrm{R}_{f}=0.33$ (4:1 hexanes:ethyl acetate, visualized by $254 \mathrm{~nm}$ light and PMA stain). ${ }^{1} \mathrm{H}$ NMR $\left(\mathrm{CDCl}_{3}, 500 \mathrm{MHz}\right): \delta 7.84(\mathrm{dd}, J=5.4,3.0 \mathrm{~Hz}, 2 \mathrm{H}), 7.71(\mathrm{dd}, J=5.4,3.1 \mathrm{~Hz}, 2 \mathrm{H}), 7.29-7.26(\mathrm{~m}, 3 \mathrm{H})$, $7.17(\mathrm{t}, J=8.8 \mathrm{~Hz}, 2 \mathrm{H}), 5.62(\mathrm{dt}, J=13.5,6.7 \mathrm{~Hz}, 1 \mathrm{H}), 5.51$ (app. dt, $J=14.4,7.4 \mathrm{~Hz}, 1 \mathrm{H}), 3.70(\mathrm{t}, J=$ $7.3 \mathrm{~Hz}, 2 \mathrm{H}), 3.30(\mathrm{~d}, J=6.6 \mathrm{~Hz}, 2 \mathrm{H}), 2.10(\mathrm{q}, J=7.2 \mathrm{~Hz}, 2 \mathrm{H}), 1.78$ (pent, $J=7.4 \mathrm{~Hz}, 2 \mathrm{H}) ;{ }^{13} \mathrm{C}$ NMR $\left(\mathrm{CDCl}_{3}, 126 \mathrm{MHz}\right): \delta 168.4,140.8,133.8,132.2,130.2,129.9,128.5,128.3,125.9,123.1,39.0,37.7$, 29.8, 28.2; FTIR (thin film): 2952, 1718, 1436, 1281, $1111 \mathrm{~cm}^{-1}$; HRMS $\mathrm{m} / z$ calculated for $\mathrm{C}_{20} \mathrm{H}_{19} \mathrm{NO}_{2} \mathrm{Na}[\mathrm{M}+\mathrm{Na}]^{+}: 328.1313$, found 328.1315 .

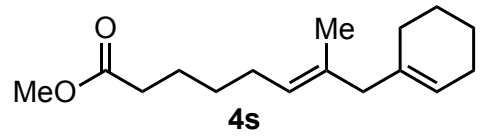

methyl $(E)-8$-(cyclohex-1-en-1-yl)-7-methyloct-6-enoate $((E)-4 s)$. The general procedure was followed using $80 \mathrm{mg}$ of $\mathrm{KF} \cdot 2 \mathrm{H}_{2} \mathrm{O}(0.85 \mathrm{mmol}), 63 \mathrm{mg}$ of 1-cyclohexenylboronic acid $3 \mathbf{j}(0.5 \mathrm{mmol}), 16$ $\mathrm{mg}$ of $\mathrm{Pd}_{2} \mathrm{dba}_{3} \cdot \mathrm{CHCl}_{3}(3.0 \mathrm{~mol} \%), 8 \mathrm{~mL}$ of DMF, and a solution of $221 \mathrm{mg}$ of $2 \mathbf{a}(0.65 \mathrm{mmol})$ and 0.21 $\mathrm{mL}$ of methyl 5-hexenoate $1 \mathbf{a}(1.5 \mathrm{mmol})$ in $2.0 \mathrm{~mL}$ of DMF. Purification by silica gel flash chromatography (97:3 hexanes:ethyl acetate) led to the isolation of $\mathbf{4 s}$ and $\mathbf{5 s}$ as a colorless oil (114 $\mathrm{mg}, 91 \%$ as a 4.9:0.44:1.0 mixture of $(E)-4 s:(Z)-4 s: 5 s$ isomers respectively), isomeric ratios were determined by GC, $\mathrm{R}_{f}=0.26$ (19:1 hexanes:ethyl acetate, visualized by PMA stain). ${ }^{1} \mathrm{H}$ NMR $\left(\mathrm{CDCl}_{3}, 500 \mathrm{MHz}\right): \delta 5.40$ $(\mathrm{m}, 1 \mathrm{H}), 5.12(\mathrm{t}, J=7.3 \mathrm{~Hz}, 1 \mathrm{H}), 3.66(\mathrm{~s}, 3 \mathrm{H}), 2.57(\mathrm{~s}, 2 \mathrm{H}), 2.31(\mathrm{t}, J=7.5 \mathrm{~Hz}, 2 \mathrm{H}), 2.00(\mathrm{~m}, 4 \mathrm{H}), 1.80$ $(\mathrm{m}, 2 \mathrm{H}), 1.67-1.51(\mathrm{~m}, 6 \mathrm{H}), 1.50(\mathrm{~s}, 3 \mathrm{H}), 1.36$ (pent, $J=7.5 \mathrm{~Hz}, 2 \mathrm{H})$; (The stereochemistry was confirmed by NOESY1D NMR spectroscopy); The following signals can be assigned to (Z)-4s: ${ }^{1} \mathrm{H}$ NMR $\left(\mathrm{CDCl}_{3}, 500 \mathrm{MHz}\right): \delta 5.20(\mathrm{t}, J=8.0 \mathrm{~Hz}, 1 \mathrm{H}), 2.62(\mathrm{~s}, 2 \mathrm{H}) ;{ }^{13} \mathrm{C} \mathrm{NMR}\left(\mathrm{CDCl}_{3}, 126 \mathrm{MHz}\right): \delta 174.5$, $136.2,133.9,125.8,122.8,51.7,49.0,34.2,29.5,27.9,27.8,25.6,24.8,23.2,22.8,15.7$; FTIR (thin film): 2926, 1741, 1436, 1170, 888, $669 \mathrm{~cm}^{-1}$; HRMS m/z calculated for $\mathrm{C}_{16} \mathrm{H}_{26} \mathrm{O}_{2} \mathrm{Na}[\mathrm{M}+\mathrm{Na}]^{+}$: 273.1831 , found 273.1826 .

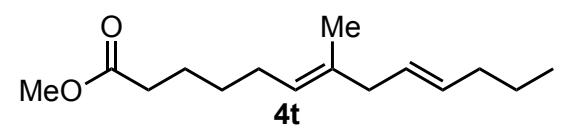

methyl $(6 E, 9 E)-7-m e t h y l t r i d e c a-6,9-d i e n o a t e ~((E)-4 t)$. The general procedure was followed using 80 $\mathrm{mg}$ of $\mathrm{KF} \cdot 2 \mathrm{H}_{2} \mathrm{O}(0.85 \mathrm{mmol}), 57 \mathrm{mg}$ of $(E)$-1-pentenylboronic acid $3 \mathbf{k}(0.5 \mathrm{mmol}), 16 \mathrm{mg}$ of $\mathrm{Pd}_{2} \mathrm{dba}_{3} \cdot \mathrm{CHCl}_{3}(3.0 \mathrm{~mol} \%), 8 \mathrm{~mL}$ of DMF, and a solution of $221 \mathrm{mg}$ of $\mathbf{2 a}(0.65 \mathrm{mmol})$ and $0.21 \mathrm{~mL}$ of methyl 5-hexenoate $1 \mathrm{a}(1.5 \mathrm{mmol})$ in $2.0 \mathrm{~mL}$ of DMF. Purification by silica gel flash chromatography (49:1 hexanes:ethyl acetate) led to the isolation of $\mathbf{4 t}$ and $\mathbf{5 t}$ as a colorless oil (52 $\mathrm{mg}, 50 \%$ as a 2.5:0.2:1.0 mixture of $(E)-\mathbf{4 t}:(Z)-\mathbf{4 t}: 5 \mathrm{t}$ isomers respectively), isomeric ratios were determined by $\mathrm{GC}, \mathrm{R}_{f}$ $=0.33$ (19:1 hexanes: ethyl acetate, visualized by PMA stain). ${ }^{1} \mathrm{H}$ NMR $\left(\mathrm{CDCl}_{3}, 500 \mathrm{MHz}\right): \delta 5.41$ (app. $\mathrm{dt}, J=15.5,9.0 \mathrm{~Hz}, 1 \mathrm{H}), 5.36$ (app. dt, $J=15.5,8.9 \mathrm{~Hz}, 1 \mathrm{H}), 5.12$ (t, $J=7.2 \mathrm{~Hz}, 1 \mathrm{H}), 3.67$ (s, 3H), 2.63 $(\mathrm{d}, J=6.0 \mathrm{~Hz}, 2 \mathrm{H}), 2.31(\mathrm{t}, J=7.5 \mathrm{~Hz}, 2 \mathrm{H}), 1.99(\mathrm{~m}, 4 \mathrm{H}), 1.63(\mathrm{~m}, 2 \mathrm{H}), 1.57(\mathrm{~s}, 3 \mathrm{H}), 1.37(\mathrm{~m}, 4 \mathrm{H})$, $0.89(\mathrm{t}, J=7.5 \mathrm{~Hz}, 3 \mathrm{H})$; (The stereochemistry was confirmed by NOESY1D NMR spectroscopy); The following signals can be assigned to $(Z)-4 \mathrm{t}:{ }^{1} \mathrm{H} \mathrm{NMR}\left(\mathrm{CDCl}_{3}, 500 \mathrm{MHz}\right): \delta 5.28(\mathrm{~m}, 2 \mathrm{H}), 2.68(\mathrm{~d}, J=$ $6.5 \mathrm{~Hz}, 2 \mathrm{H}) ;{ }^{13} \mathrm{C} \mathrm{NMR}\left(\mathrm{CDCl}_{3}, 126 \mathrm{MHz}\right): \delta 174.5,135.0,131.9,128.6,124.6,51.7,43.2,34.9,34.3$, 
29.5, 27.8, 24.9, 22.9, 16.2, 13.9; FTIR (thin film): 2956, 1741, 1435, 1064, 968, $739 \mathrm{~cm}^{-1}$; HRMS m/z calculated for $\mathrm{C}_{15} \mathrm{H}_{26} \mathrm{O}_{2} \mathrm{Na}[\mathrm{M}+\mathrm{Na}]^{+}:$261.1831, found 261.1829.

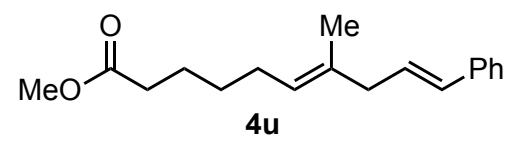

methyl $(6 E, 9 E)-7-m e t h y l-10-p h e n y l d e c a-6,9-d i e n o a t e ~((E)-4 u)$. The general procedure was followed using $80 \mathrm{mg}$ of $\mathrm{KF} \bullet 2 \mathrm{H}_{2} \mathrm{O}(0.85 \mathrm{mmol}), 74 \mathrm{mg}$ of $(E)$-(2-phenylvinyl)boronic acid 31 ( $\left.0.5 \mathrm{mmol}\right), 16 \mathrm{mg}$ of $\mathrm{Pd}_{2} \mathrm{dba}_{3} \cdot \mathrm{CHCl}_{3}(3.0 \mathrm{~mol} \%), 8 \mathrm{~mL}$ of DMF, and a solution of $221 \mathrm{mg}$ of $\mathbf{2 a}(0.65 \mathrm{mmol})$ and $0.21 \mathrm{~mL}$ of methyl 5-hexenoate 1a $(1.5 \mathrm{mmol})$ in $2.0 \mathrm{~mL}$ of DMF. Purification by silica gel flash chromatography (19:1 hexanes:ethyl acetate) led to the isolation of $\mathbf{4 u}$ and $\mathbf{5 u}$ as a colorless oil (103 $\mathrm{mg}, 76 \%$ as a 4.1:0.41:1.0 mixture of $(E)-\mathbf{4 u}:(Z)-\mathbf{4 u}: 5 \mathbf{u}$ isomers respectively), isomeric ratios were determined by GC, $\mathrm{R}_{f}=0.22$ (19:1 hexanes:ethyl acetate, visualized by $254 \mathrm{~nm}$ light and PMA stain). ${ }^{1} \mathrm{H} \mathrm{NMR}\left(\mathrm{CDCl}_{3}\right.$, $400 \mathrm{MHz}): \delta 7.35(\mathrm{~m}, 2 \mathrm{H}), 7.29(\mathrm{~m}, 2 \mathrm{H}), 7.19(\mathrm{~m}, 1 \mathrm{H}), 6.38(\mathrm{~d}, J=16.0 \mathrm{~Hz}, 1 \mathrm{H}), 6.19(\mathrm{dt}, J=15.6,6.8$ $\mathrm{Hz}, 1 \mathrm{H}), 5.21$ (t, $J=7.2 \mathrm{~Hz}, 1 \mathrm{H}), 3.66(\mathrm{~s}, 3 \mathrm{H}), 2.86(\mathrm{~d}, J=7.2 \mathrm{~Hz}, 2 \mathrm{H}), 2.32$ (t, J=7.6 Hz, 2H), 2.04 (q, $J=7.6 \mathrm{~Hz}, 2 \mathrm{H}), 1.63(\mathrm{~m}, 5 \mathrm{H}), 1.38$ (pent, $J=7.6 \mathrm{~Hz}, 2 \mathrm{H}$ ); (The stereochemistry was confirmed by NOESY1D NMR spectroscopy); The following signals can be assigned to $(Z)-4 \mathbf{u}:{ }^{1} \mathrm{H}$ NMR $\left(\mathrm{CDCl}_{3}\right.$, $400 \mathrm{MHz}): \delta 6.13(\mathrm{~m}, 1 \mathrm{H}), 3.65(\mathrm{~s}, 3 \mathrm{H}), 2.91(\mathrm{~d}, J=6.4 \mathrm{~Hz}, 2 \mathrm{H}) 1.72(\mathrm{~s}, 3 \mathrm{H}) ;{ }^{13} \mathrm{C} \mathrm{NMR}\left(\mathrm{CDCl}_{3}, 126\right.$ $\mathrm{MHz}): \delta 174.4,137.9,134.2,131.1,129.2,128.7,127.1,126.2,125.5,51.7,43.4,34.2,29.5,27.9,24.9$, 16.4; FTIR (thin film): 2931, 1736, 1435, 965, 738, $692 \mathrm{~cm}^{-1}$; HRMS m/z calculated for $\mathrm{C}_{18} \mathrm{H}_{24} \mathrm{O}_{2} \mathrm{Na}$ $[\mathrm{M}+\mathrm{Na}]^{+}: 295.1674$, found 295.1667 .

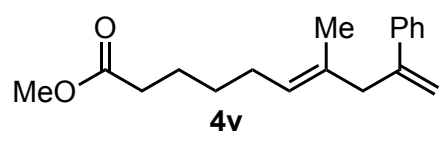

methyl $(E)-7-m e t h y l-9-p h e n y l d e c a-6,9-d i e n o a t e ~((E)-4 v)$. The general procedure was followed using $80 \mathrm{mg}$ of $\mathrm{KF} \cdot 2 \mathrm{H}_{2} \mathrm{O}(0.85 \mathrm{mmol}), 74 \mathrm{mg}$ of (1-phenylvinyl)boronic acid $3 \mathrm{~m}(0.5 \mathrm{mmol}), 16 \mathrm{mg}$ of $\mathrm{Pd}_{2} \mathrm{dba}_{3} \cdot \mathrm{CHCl}_{3}(3.0 \mathrm{~mol} \%), 8 \mathrm{~mL}$ of DMF, and a solution of $221 \mathrm{mg}$ of $\mathbf{2 a}(0.65 \mathrm{mmol})$ and $0.21 \mathrm{~mL}$ of methyl 5-hexenoate 1a $(1.5 \mathrm{mmol})$ in $2.0 \mathrm{~mL}$ of DMF. Purification by silica gel flash chromatography (99:1 hexanes: $\mathrm{Et}_{2} \mathrm{O} \rightarrow$ 19:1 hexanes:ethyl acetate) led to the isolation of $\mathbf{4} \mathbf{v}$ and $\mathbf{5 v}$ as a colorless oil (51 $\mathrm{mg}, 37 \%$ as a 13.2:1.3:1.0 mixture of $(E)-\mathbf{4 v}:(Z)-\mathbf{4 v}: \mathbf{5 v}$ isomers respectively), isomeric ratios were determined by GC, $\mathrm{R}_{f}=0.26$ (19:1 hexanes:ethyl acetate, visualized by $254 \mathrm{~nm}$ light and PMA stain). ${ }^{1} \mathrm{H}$ $\operatorname{NMR}\left(\mathrm{CDCl}_{3}, 500 \mathrm{MHz}\right): \delta 7.40(\mathrm{~m}, 2 \mathrm{H}), 7.29(\mathrm{~m}, 2 \mathrm{H}), 7.23(\mathrm{~m}, 1 \mathrm{H}), 5.38(\mathrm{~d}, J=1.5 \mathrm{~Hz}, 1 \mathrm{H}), 5.20(\mathrm{t}$, $J=7.3 \mathrm{~Hz}, 1 \mathrm{H}), 5.06(\mathrm{~d}, J=1.5 \mathrm{~Hz}, 1 \mathrm{H}), 3.66(\mathrm{~s}, 3 \mathrm{H}), 3.16(\mathrm{~s}, 2 \mathrm{H}), 2.24(\mathrm{t}, J=7.5 \mathrm{~Hz}, 2 \mathrm{H}), 1.99(\mathrm{q}, J=$ $7.5 \mathrm{~Hz}, 2 \mathrm{H}$ ), $1.52(\mathrm{~m}, 5 \mathrm{H}), 1.30$ (pent, $J=7.5 \mathrm{~Hz}, 2 \mathrm{H}$ ); (The stereochemistry was confirmed by NOESY1D NMR spectroscopy); ${ }^{13} \mathrm{C}$ NMR $\left(\mathrm{CDCl}_{3}, 126 \mathrm{MHz}\right): \delta 174.4,146.5,133.2,128.3,127.4$, 127.1, 126.4, 114.1, 51.7, 45.9, 34.2, 29.3, 27.8, 24.6, 16.1; FTIR (thin film): 2925, 1739, 1441, 1171, 897, 778, $707 \mathrm{~cm}^{-1}$; HRMS $m / z$ calculated for $\mathrm{C}_{18} \mathrm{H}_{24} \mathrm{O}_{2} \mathrm{Na}[\mathrm{M}+\mathrm{Na}]^{+}: 295.1674$, found 295.1669. 


\section{References}

1. Zalesskiy, S. S.; Ananikov, V. P. Organometallics 2012, 31, 2302.

2. Li, T.-S.; Li, J.-T.; Li, H.-Z. J. Chromatogr. A 1995, 715, 372.

3. Williams, C. M.; Mander, L. N. Tetrahedron 2001, 57, 425.

4. Lyapkalo, I. M.; Webel, M.; Reißig, H.-U. Eur. J. Org. Chem. 2001, 2001, 4189.

5. Innitzer, A.; Brecker, L.; Mulzer, J. Org. Lett. 2007, 9, 4431.

6. Tranchant, M.-J.; Dalla, V.; Jabin, I.; Decroix, B. Tetrahedron 2002, 58, 8425. 


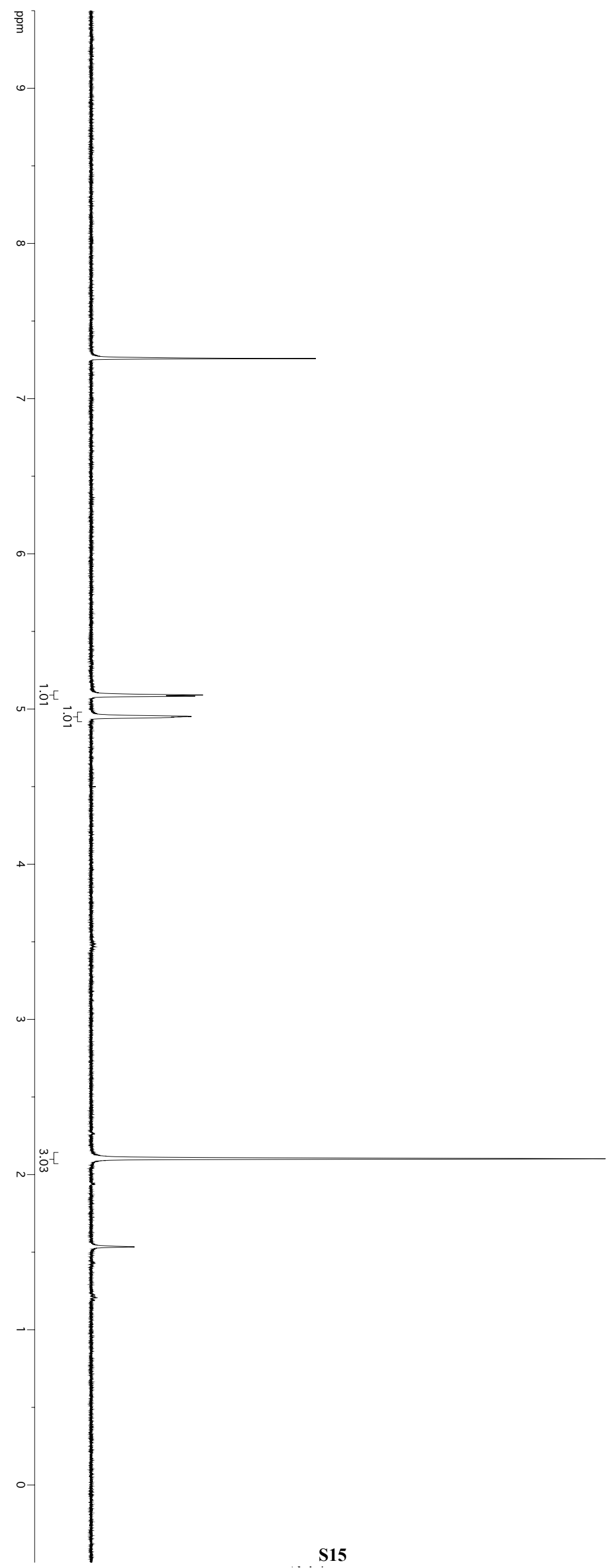

$\approx \frac{2}{0}-\frac{3}{0}$ 


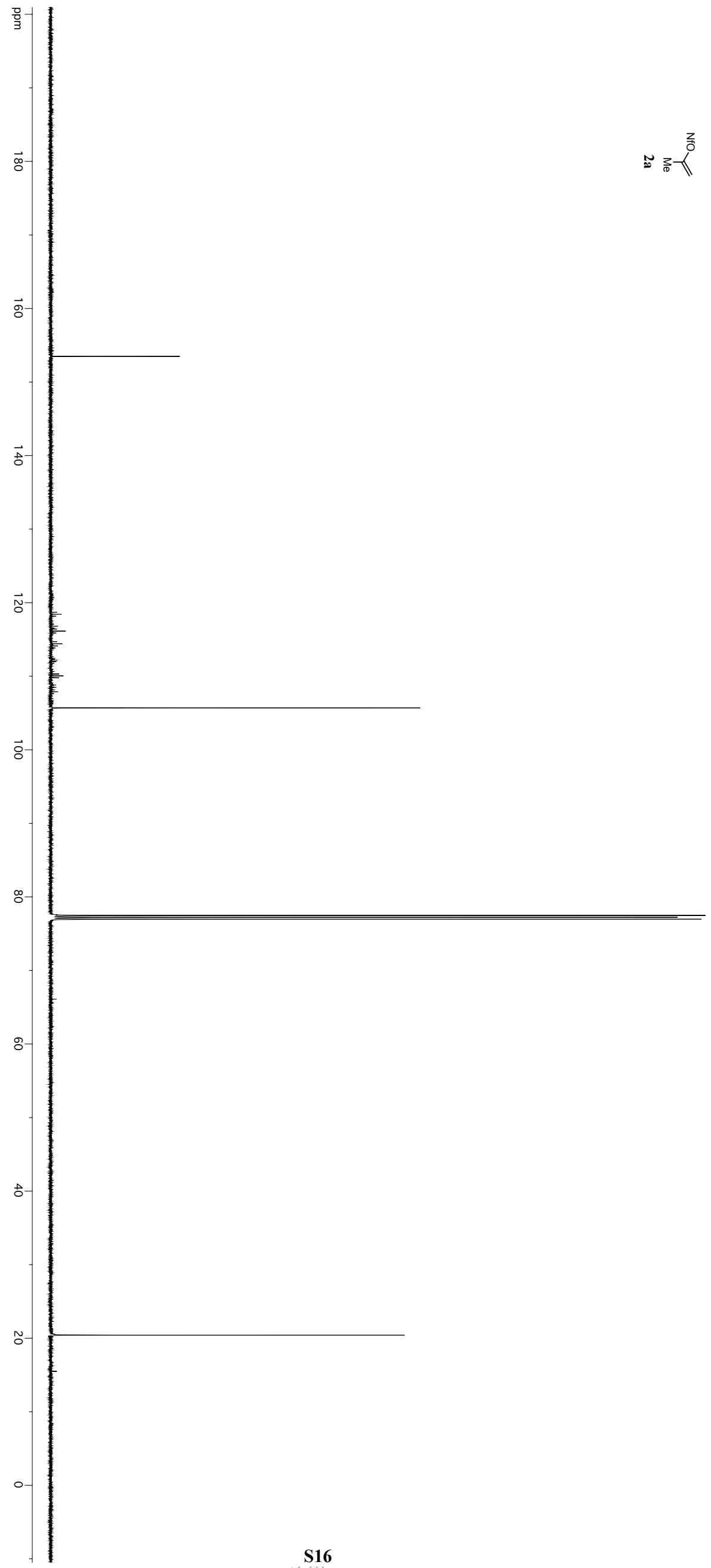




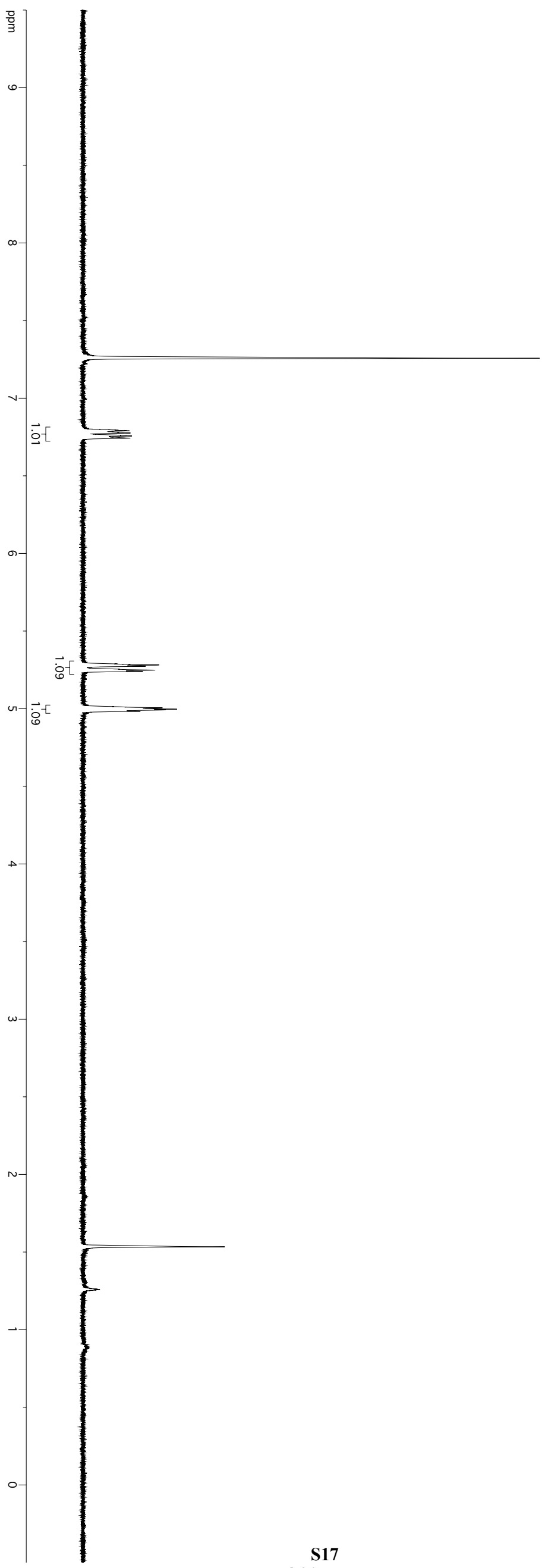




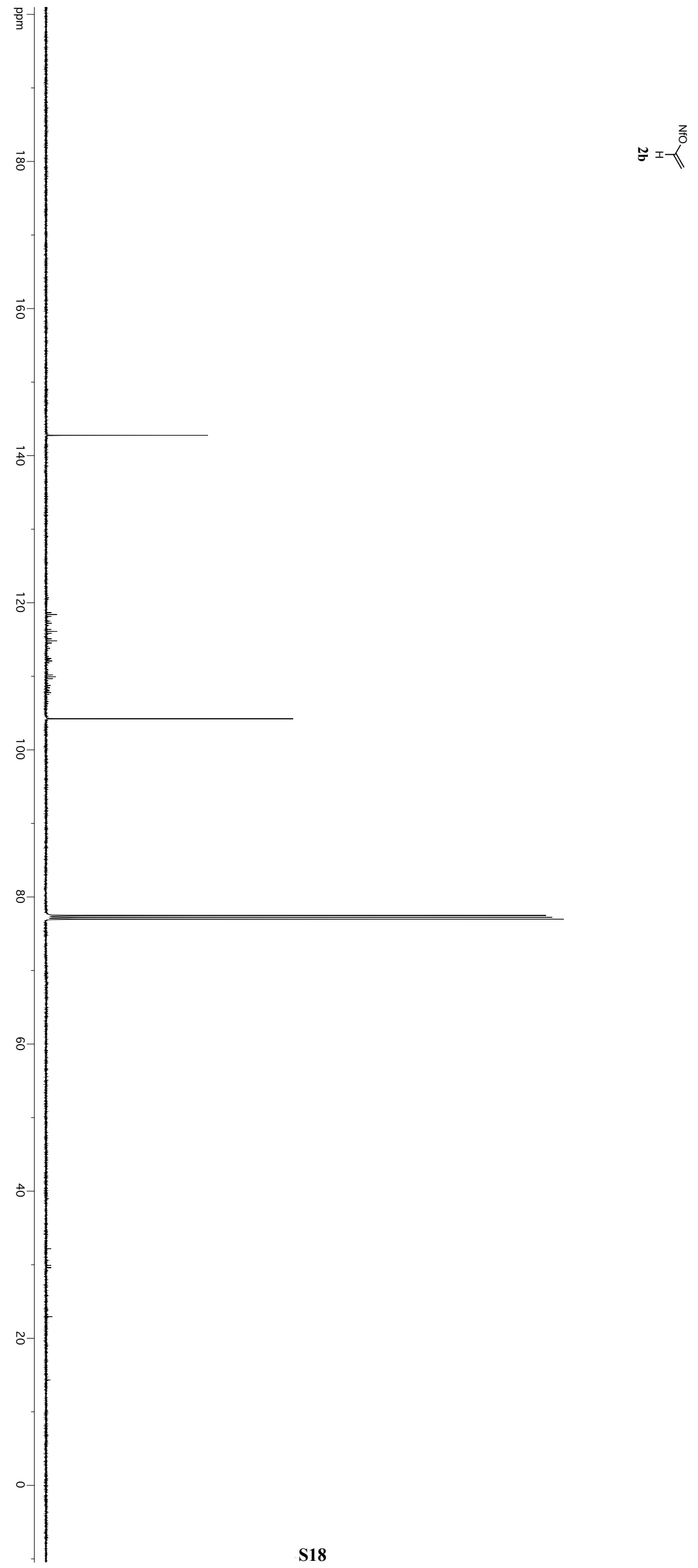




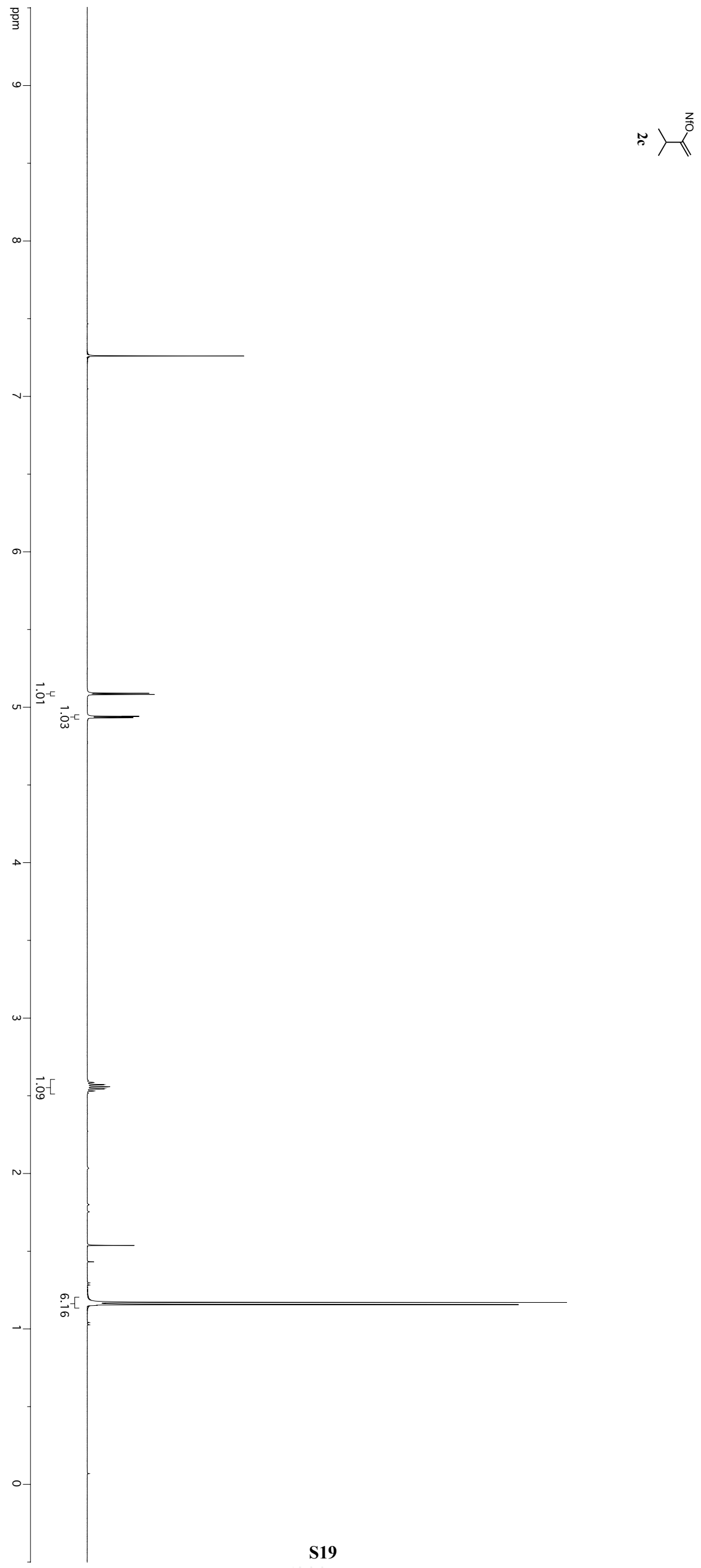




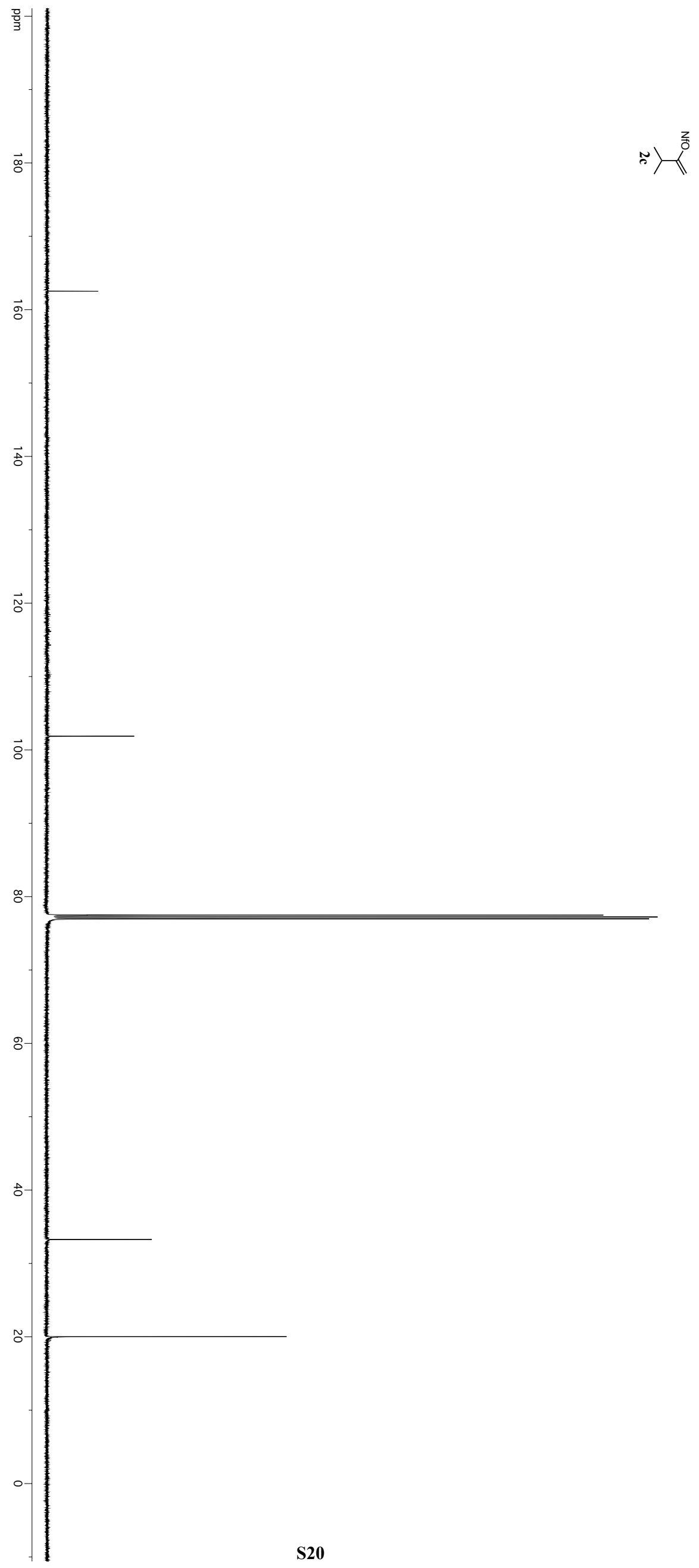




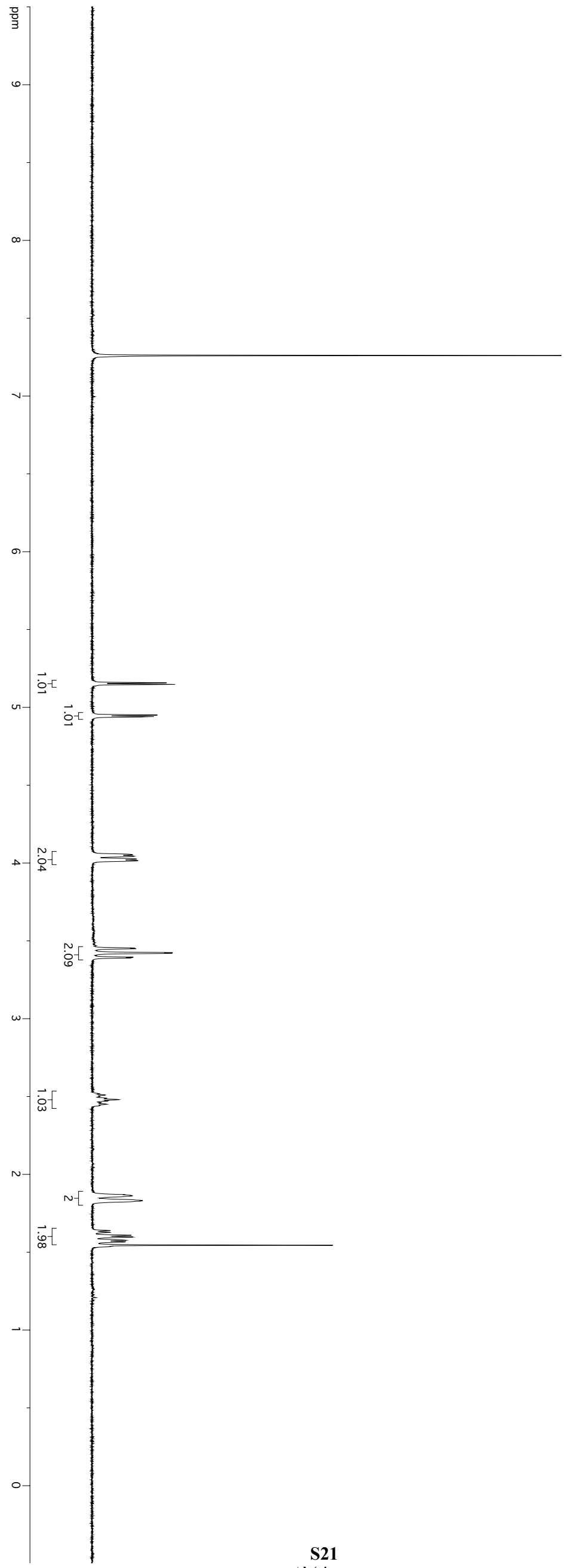




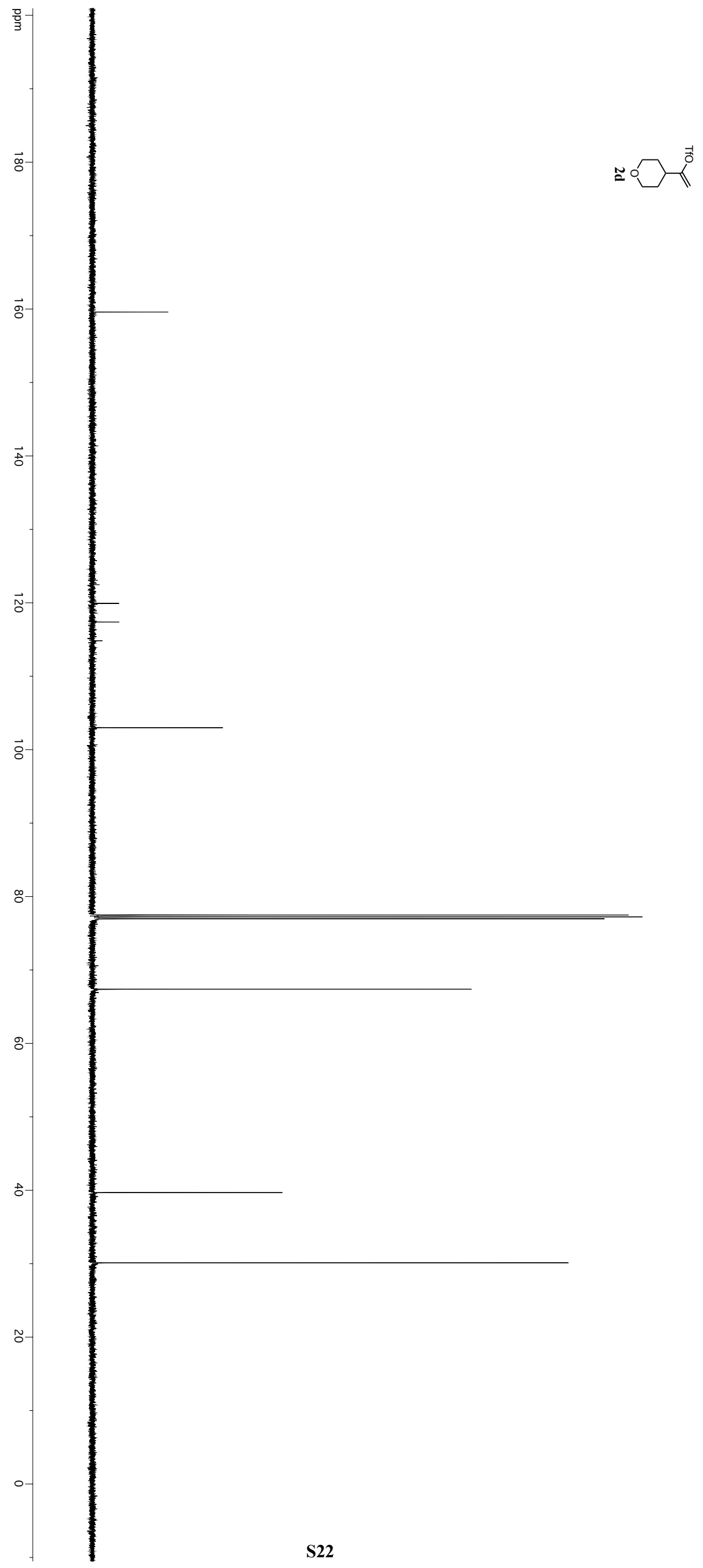




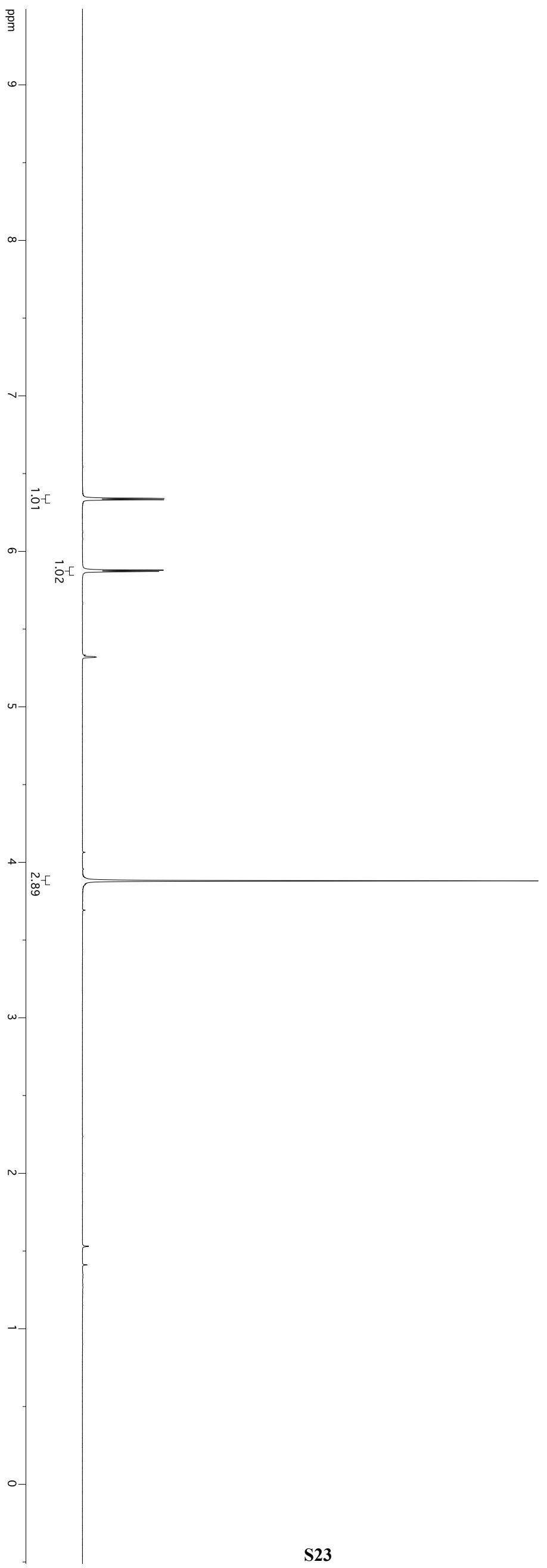

赵 


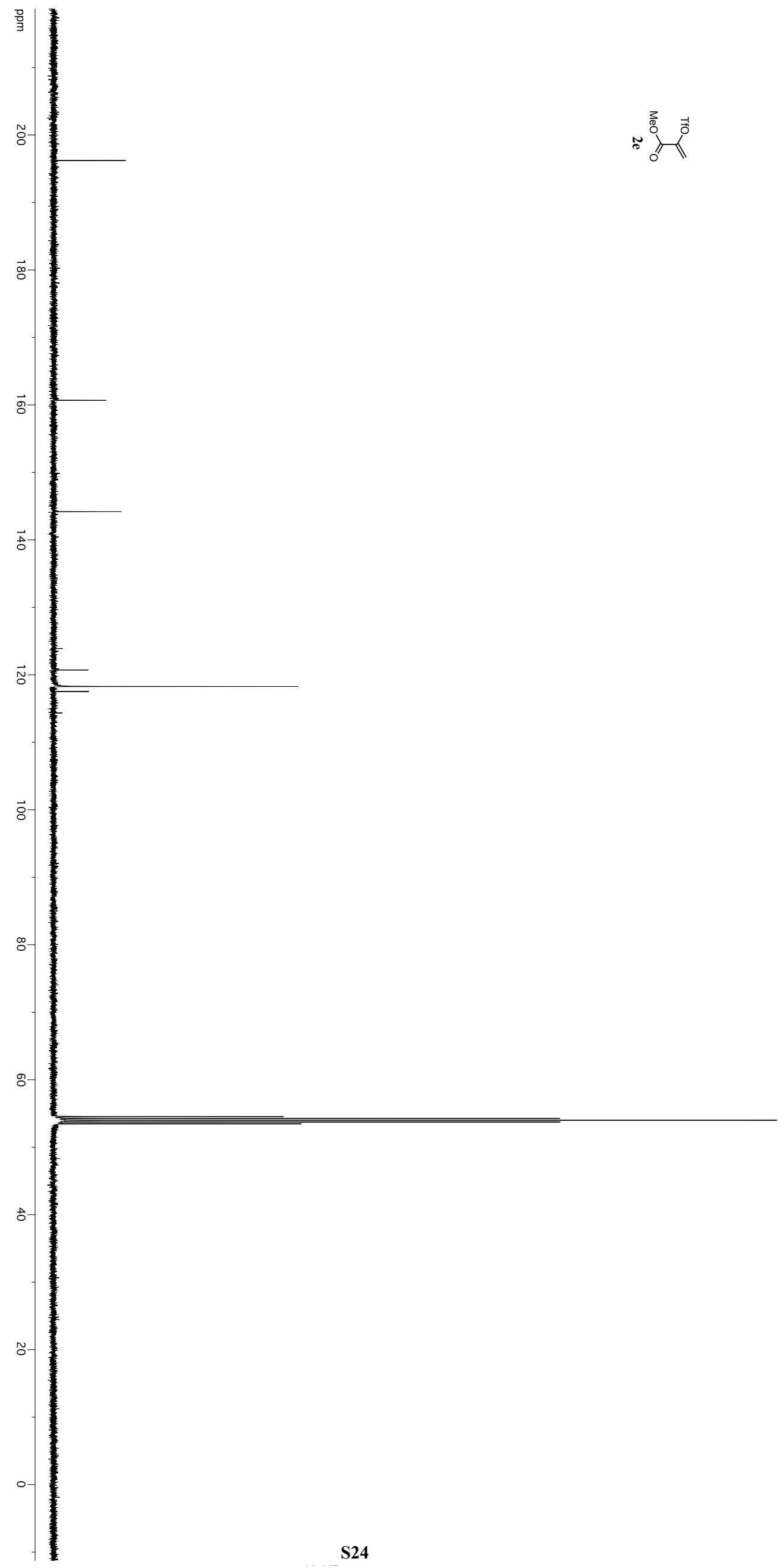




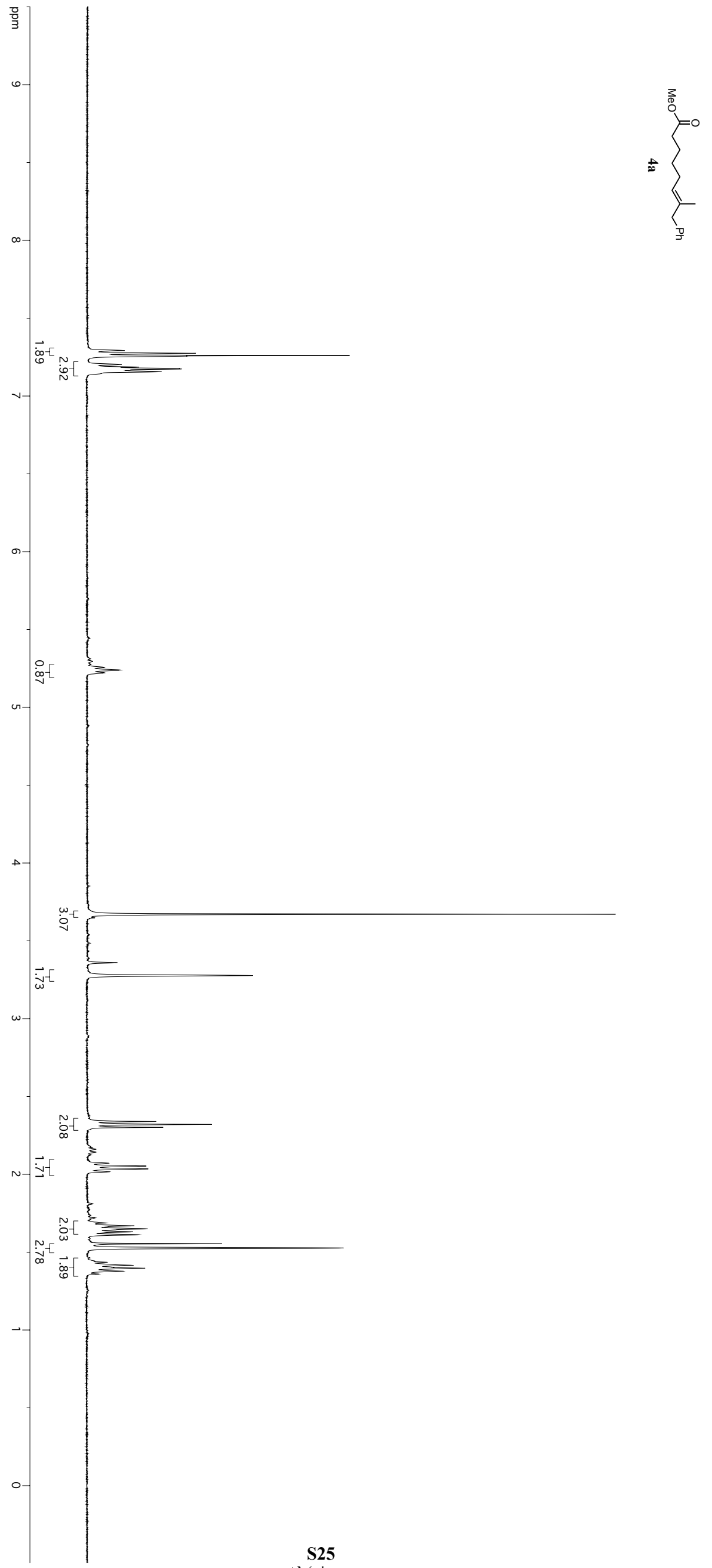




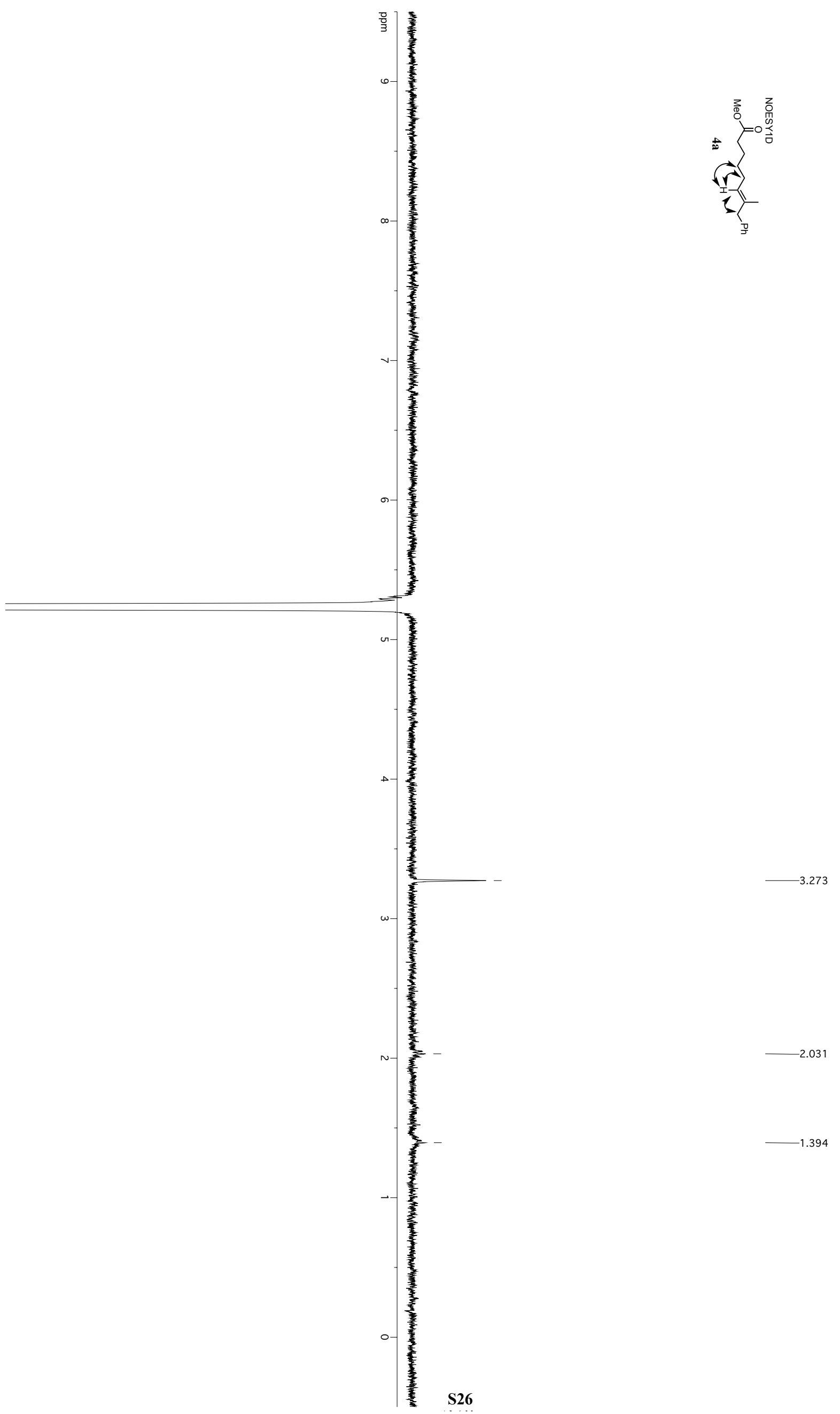




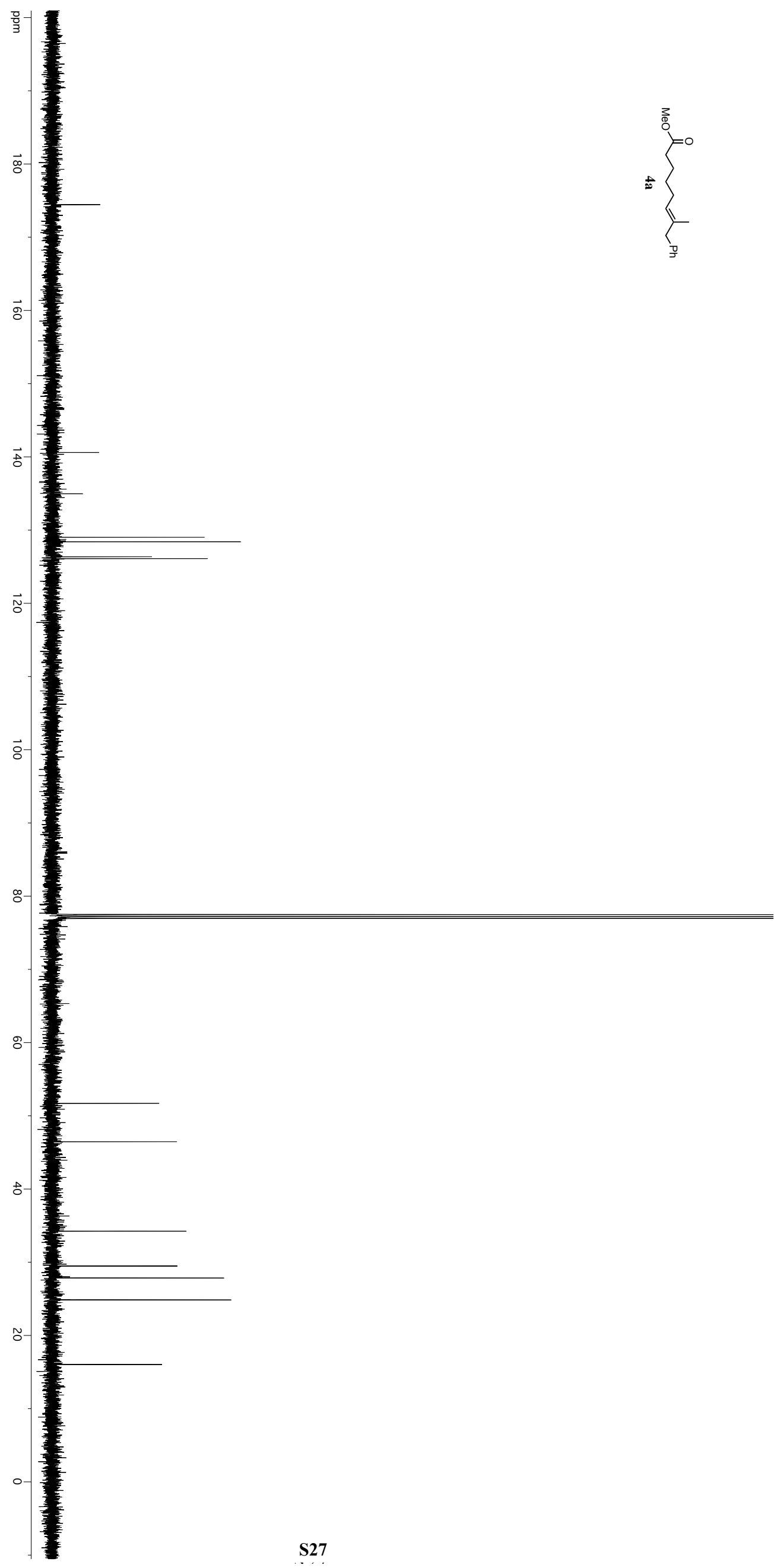



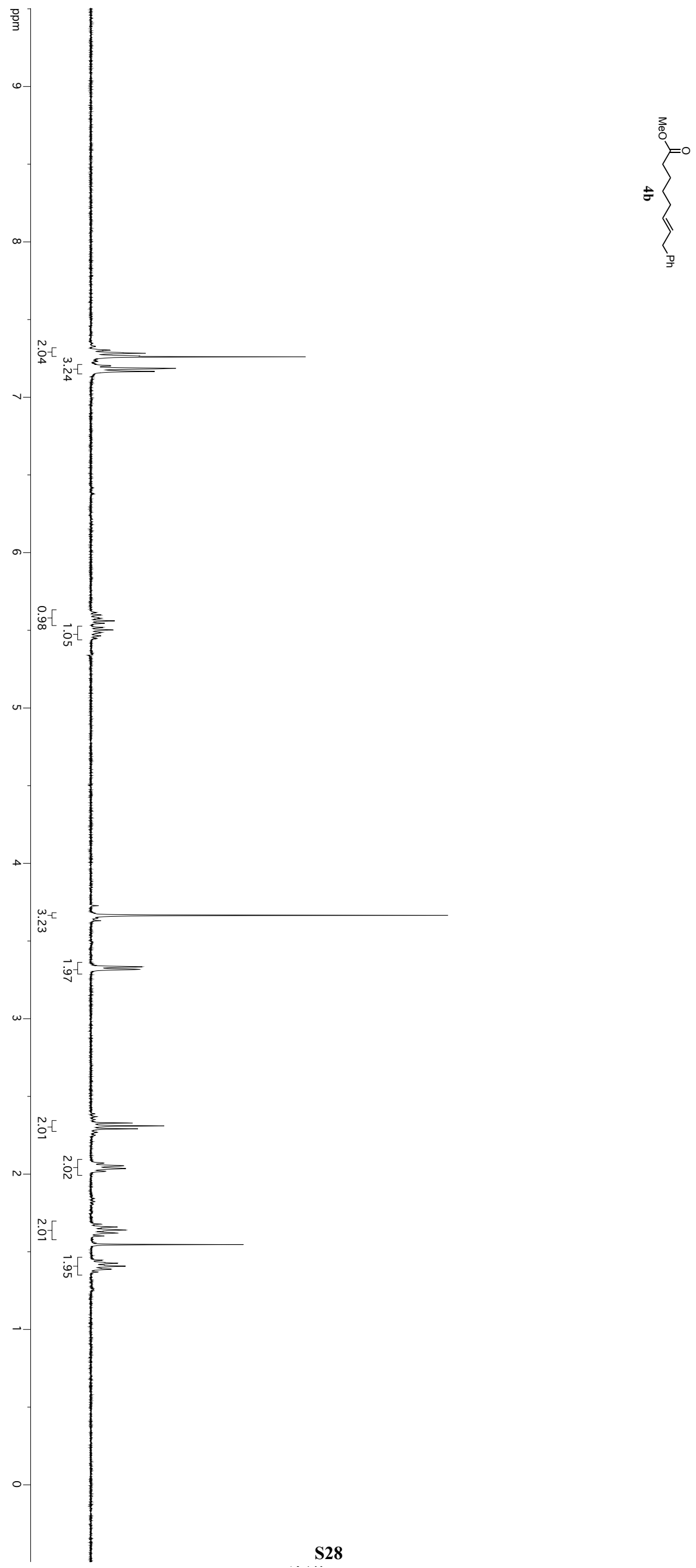


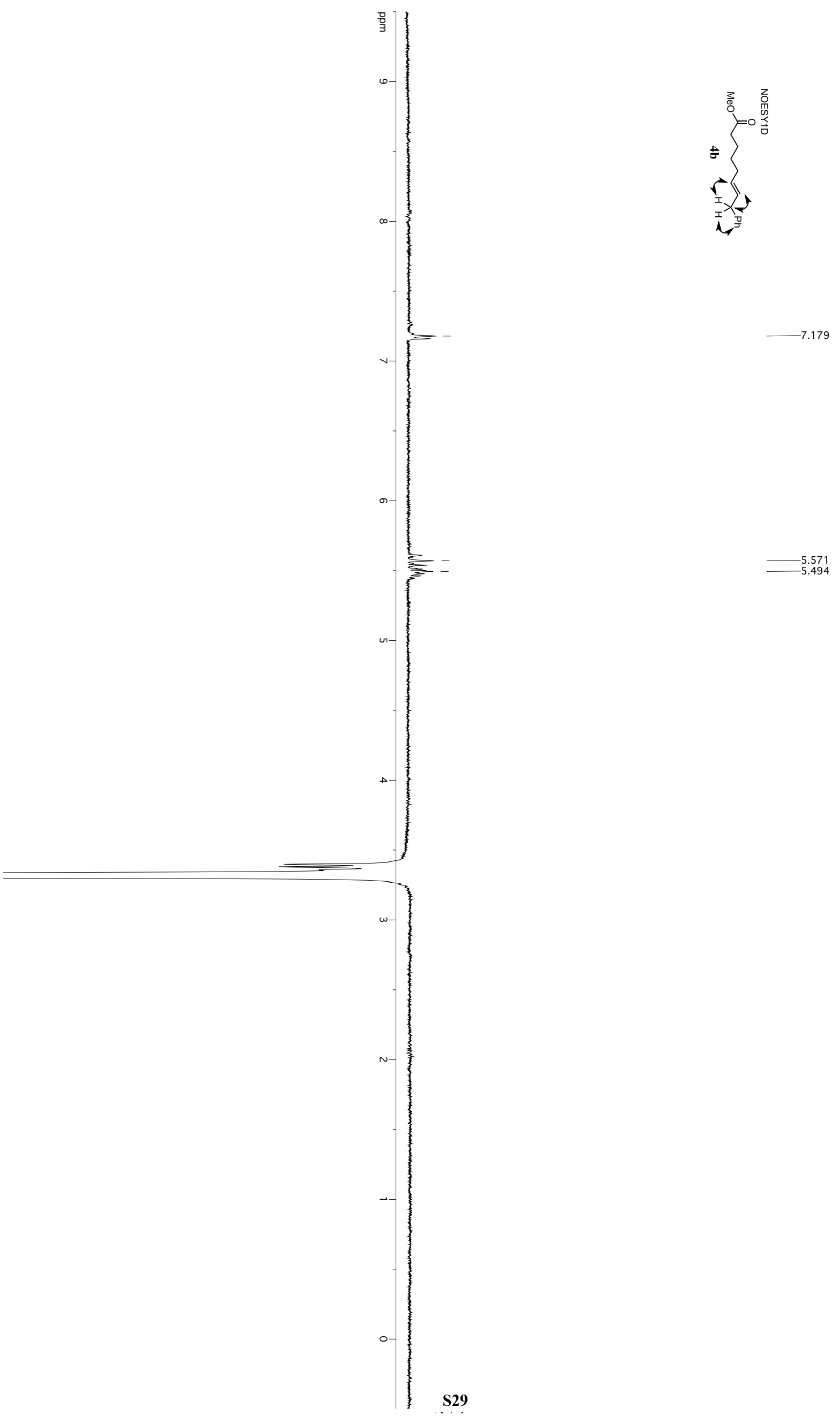




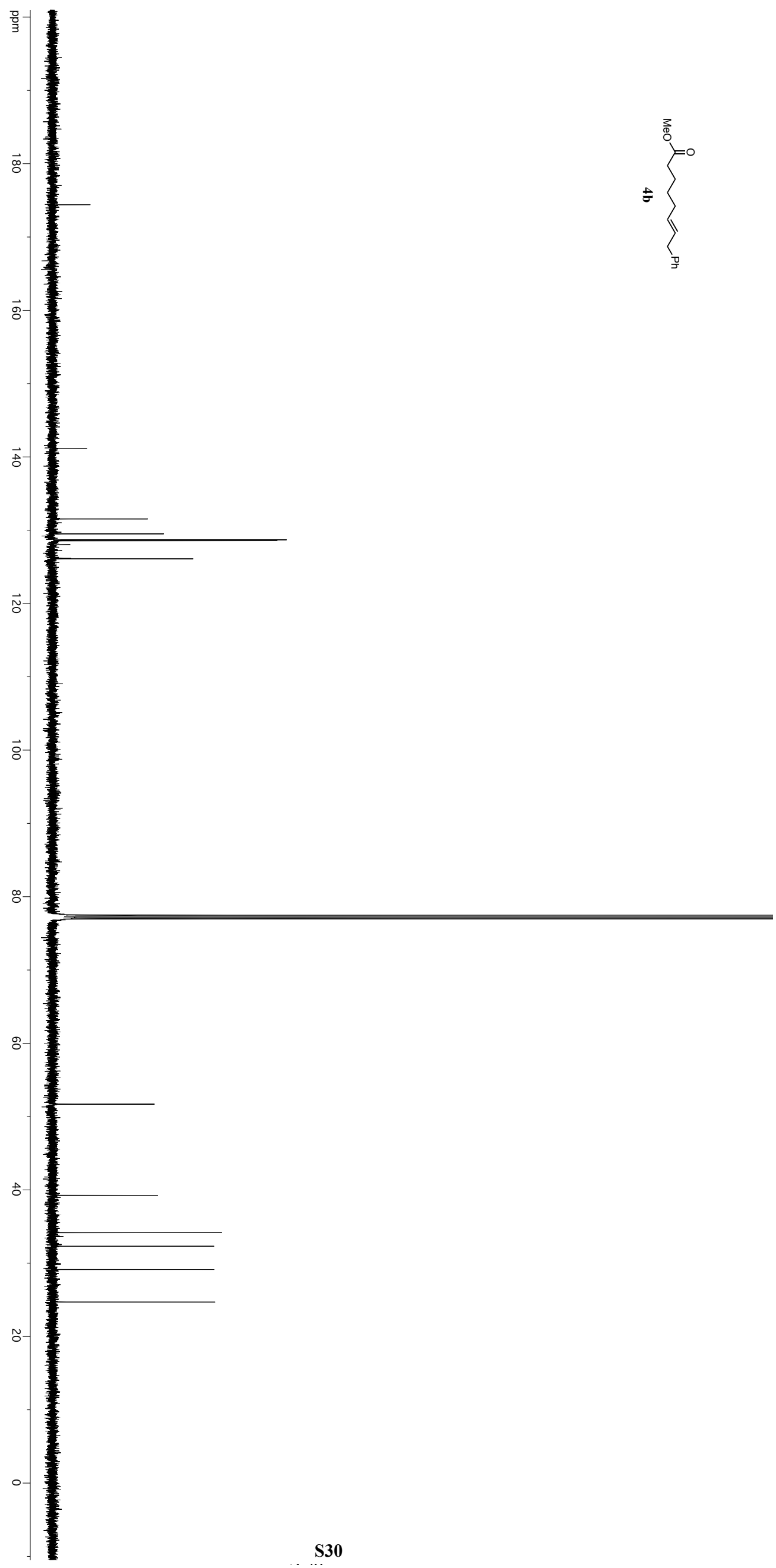



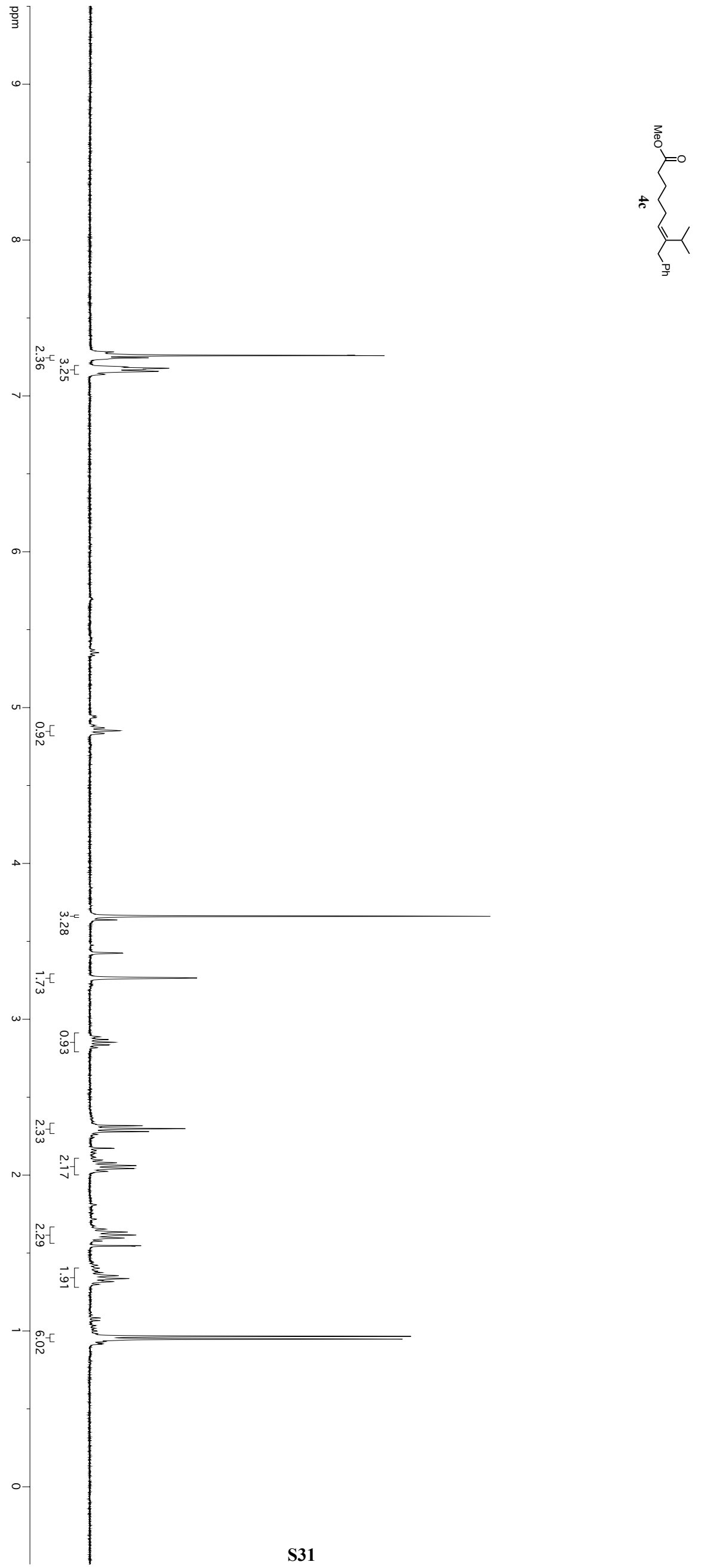


$$
4
$$



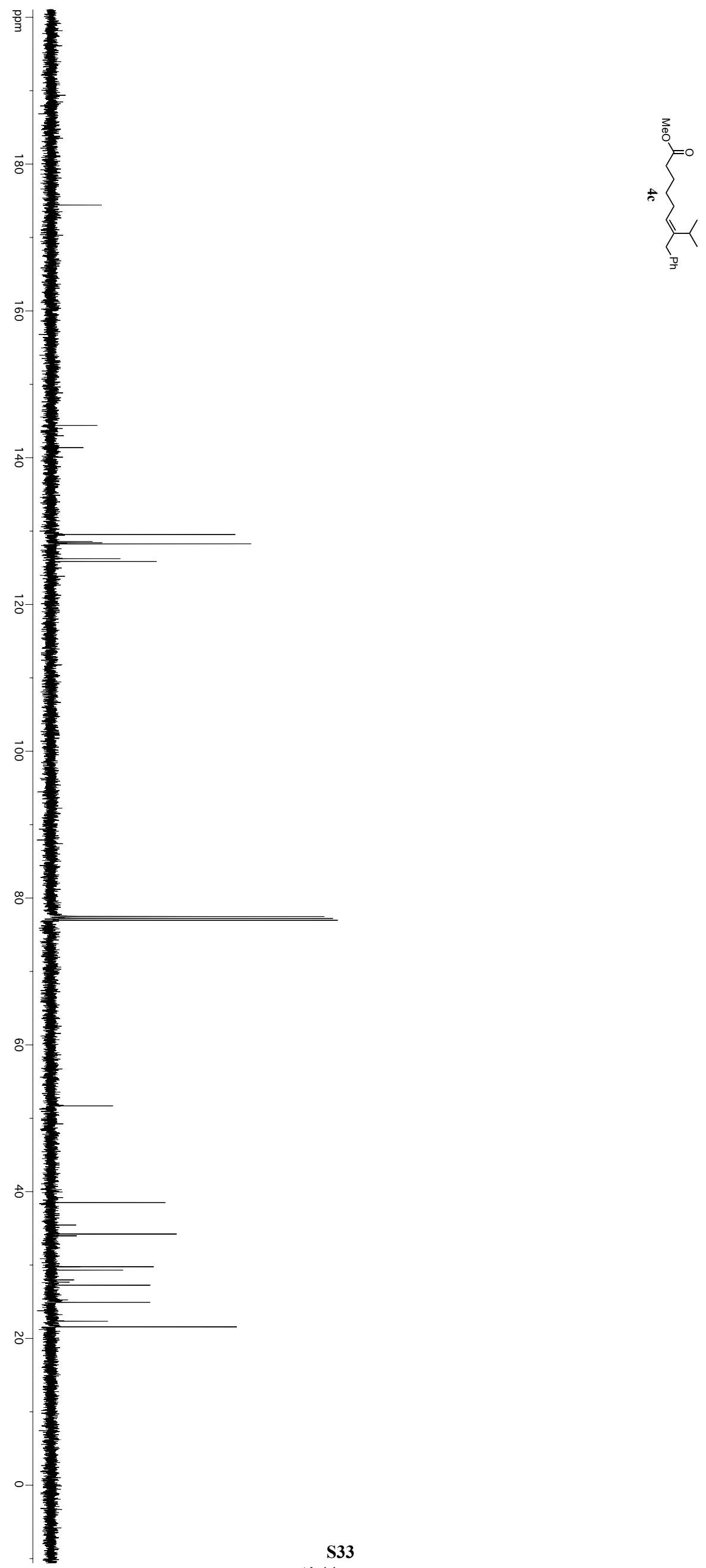


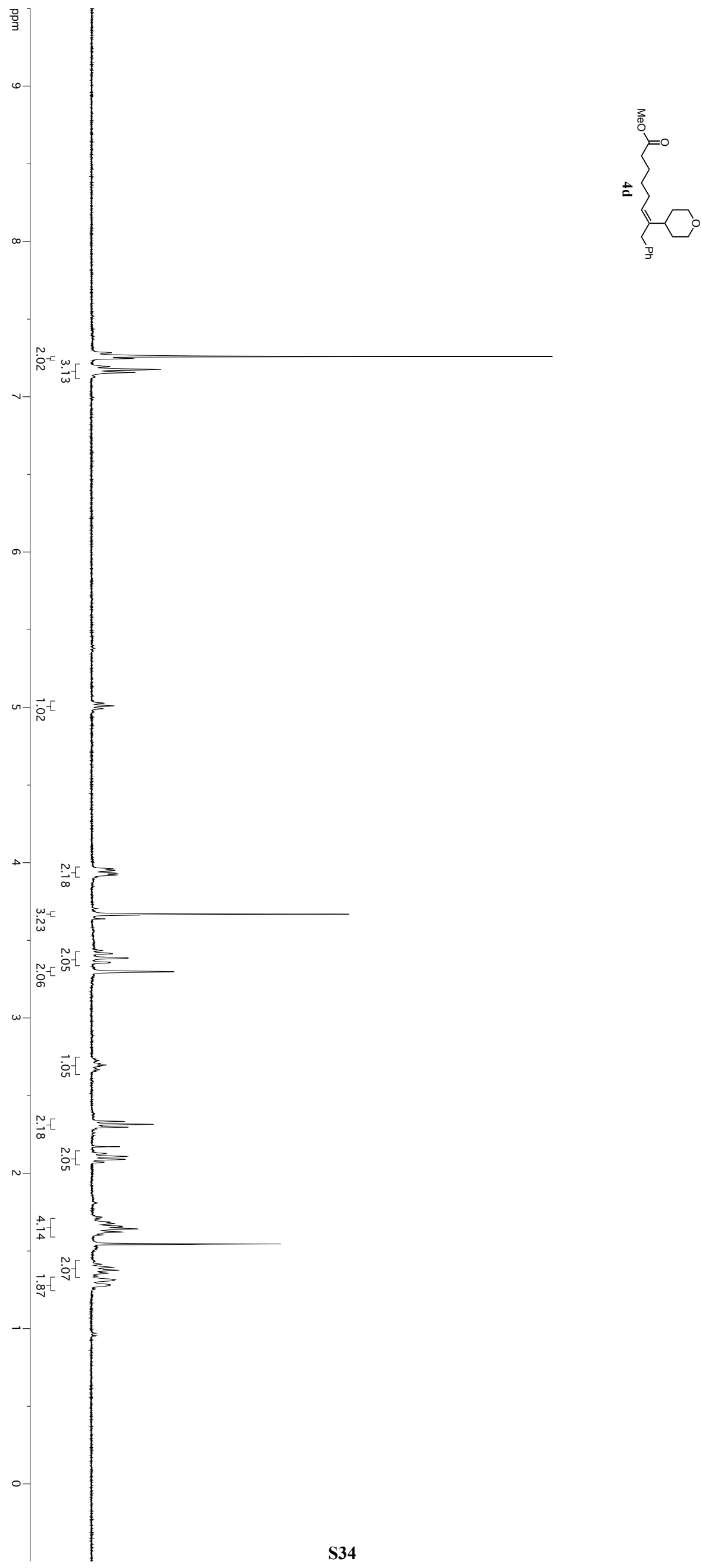



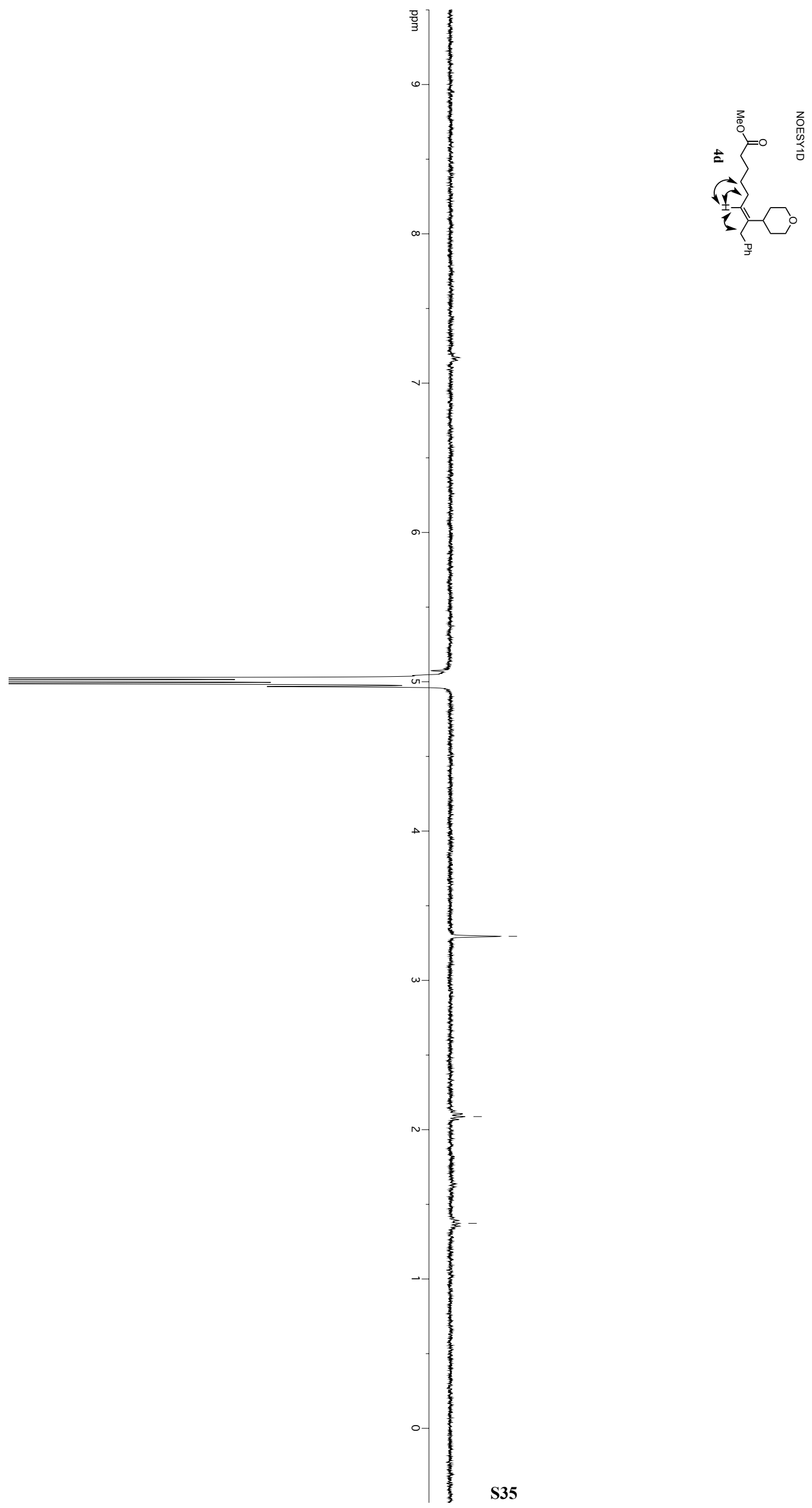

$-3.295$

$-2.088$

$-1.373$ 


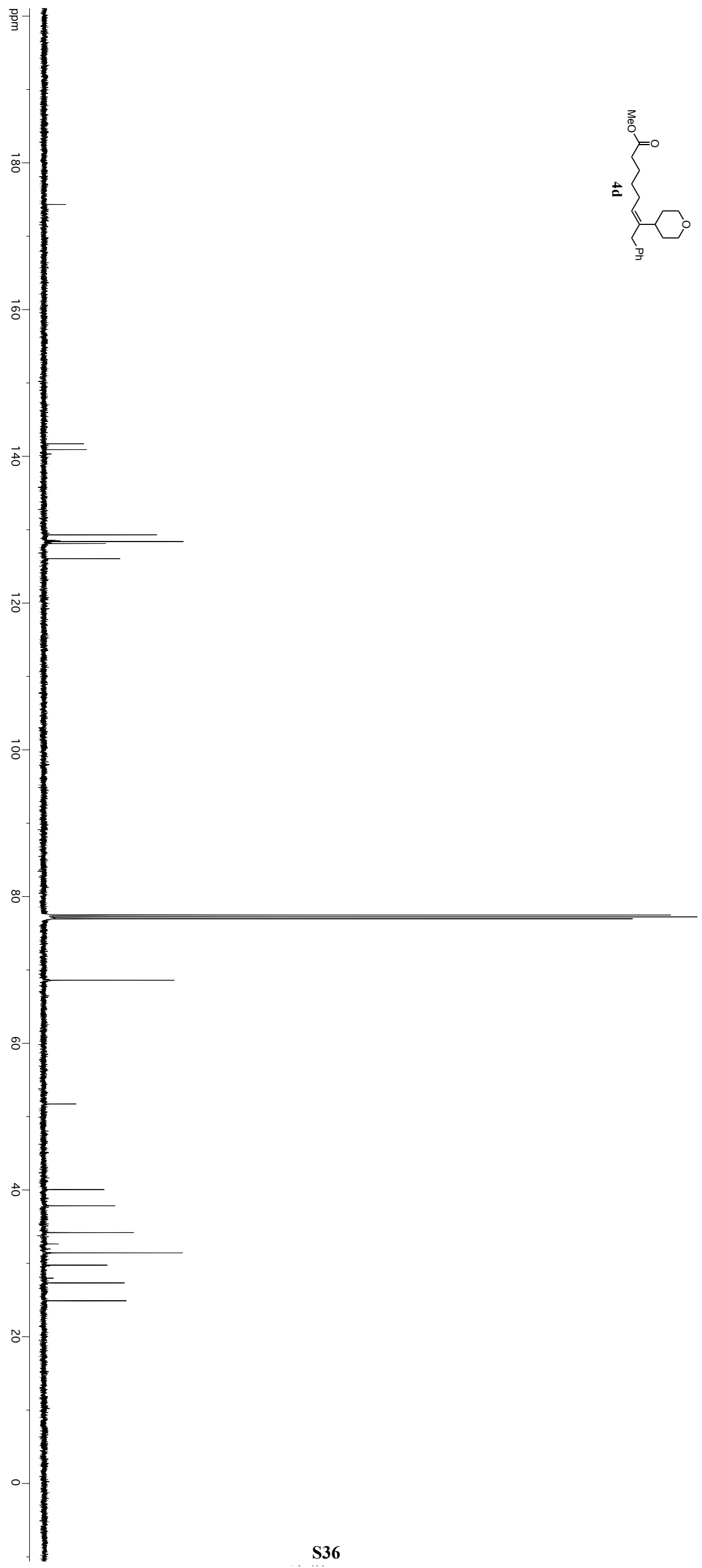



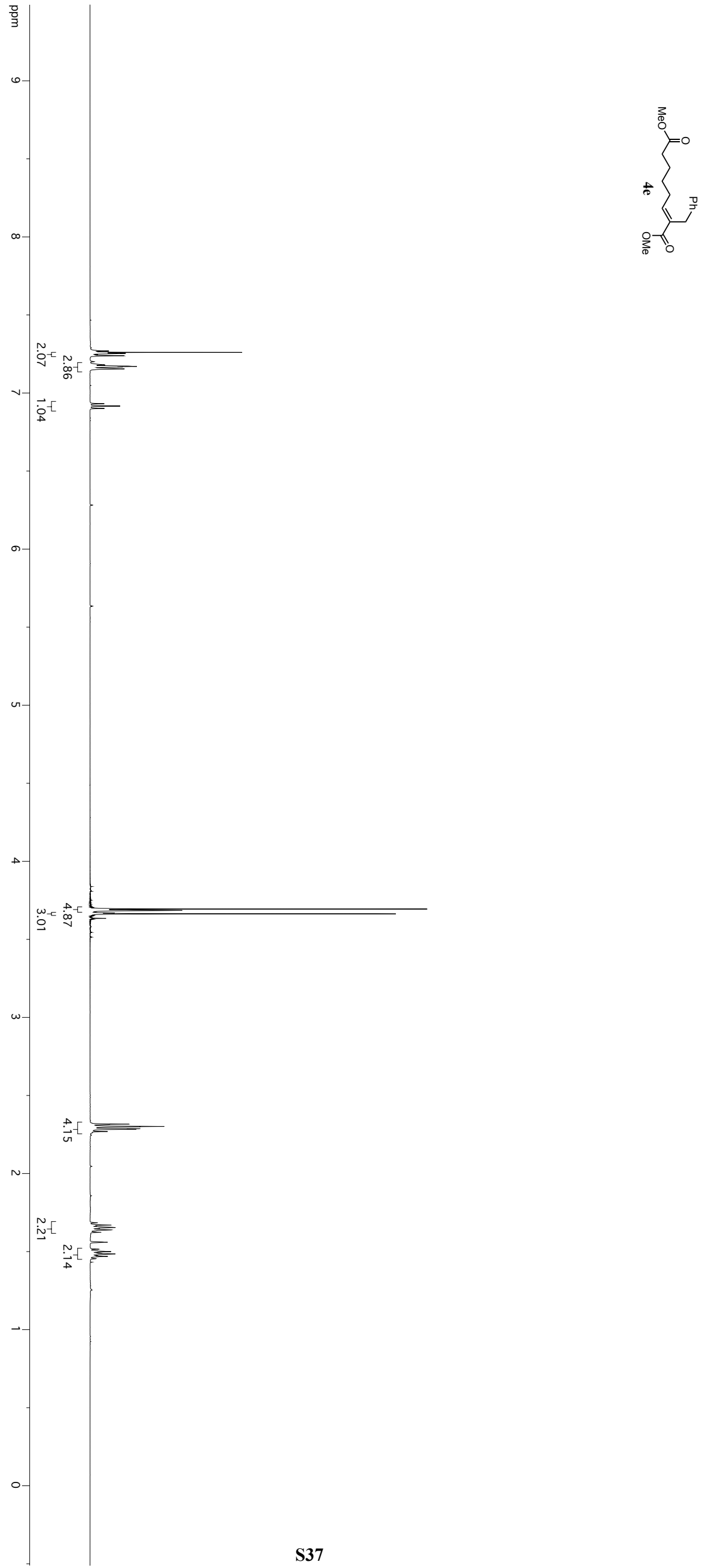

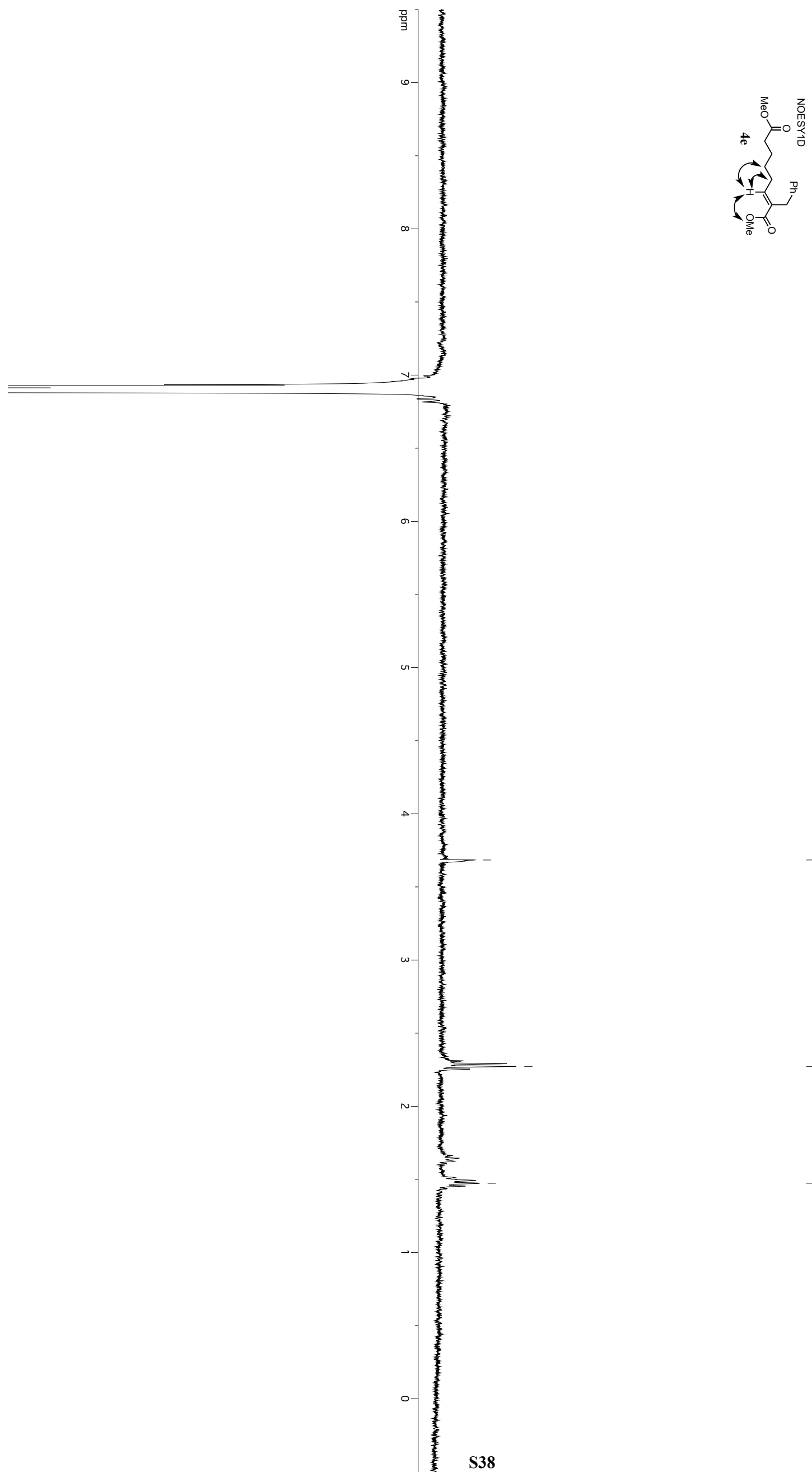

$-3.684$

$-2.272$

$-1.473$ 


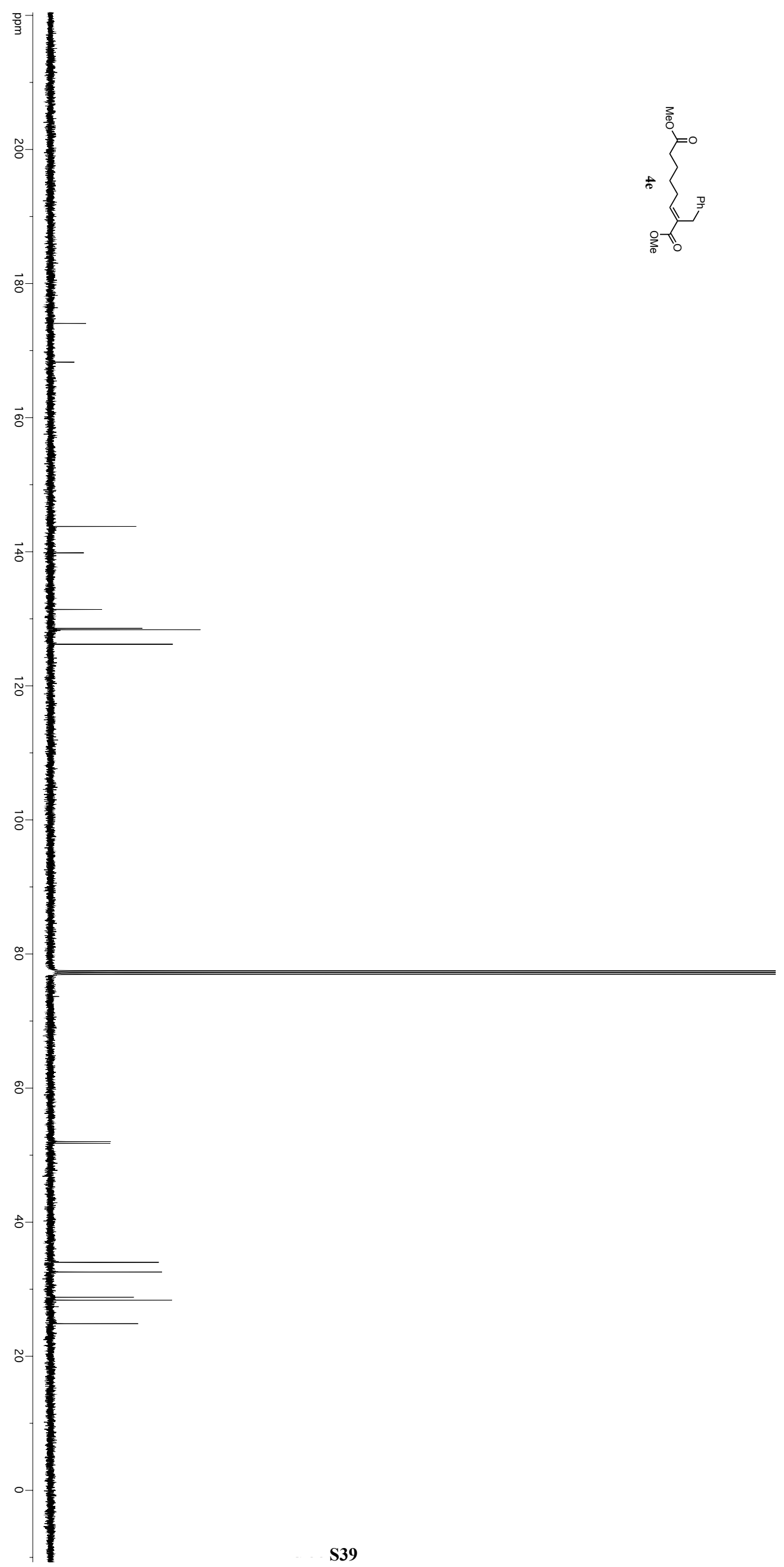




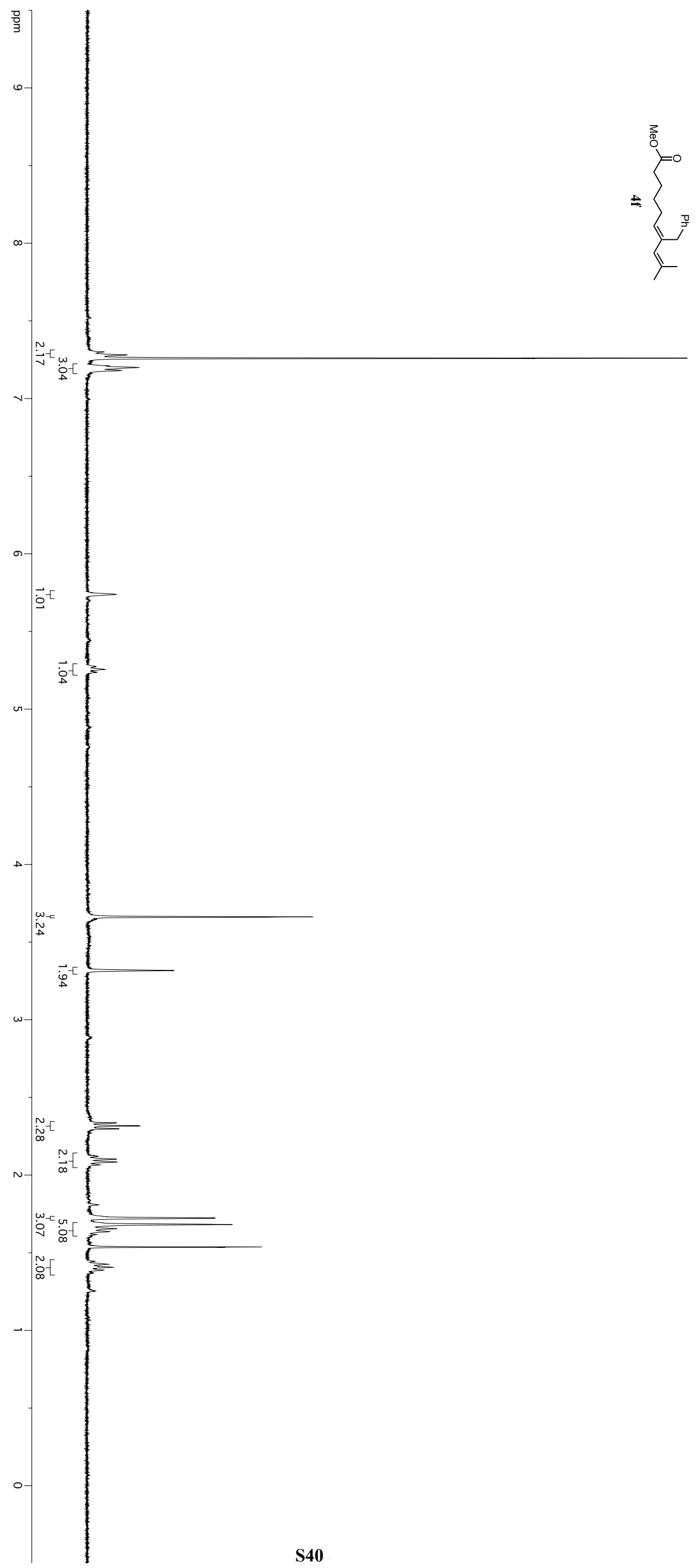




$$
1
$$




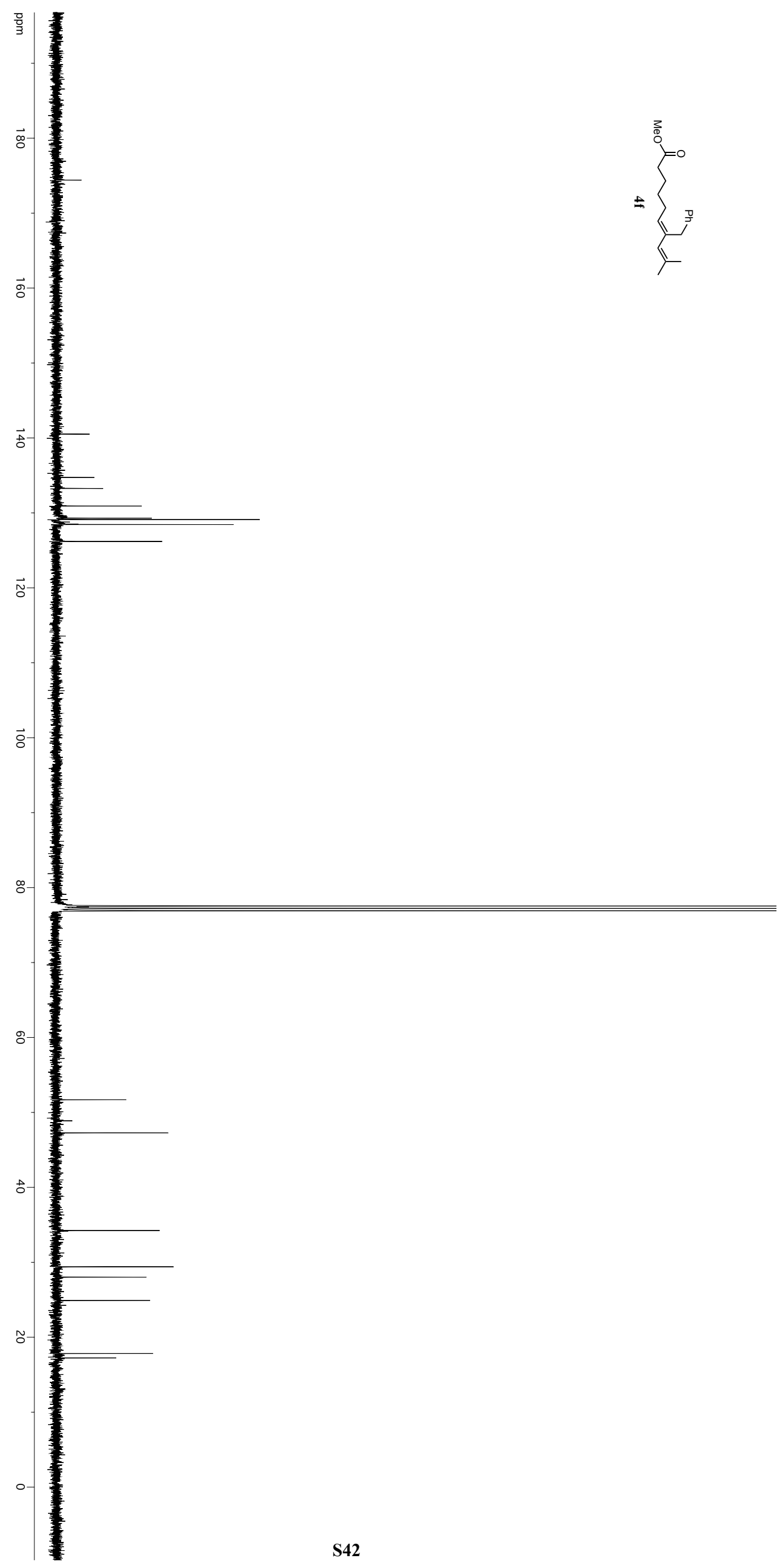




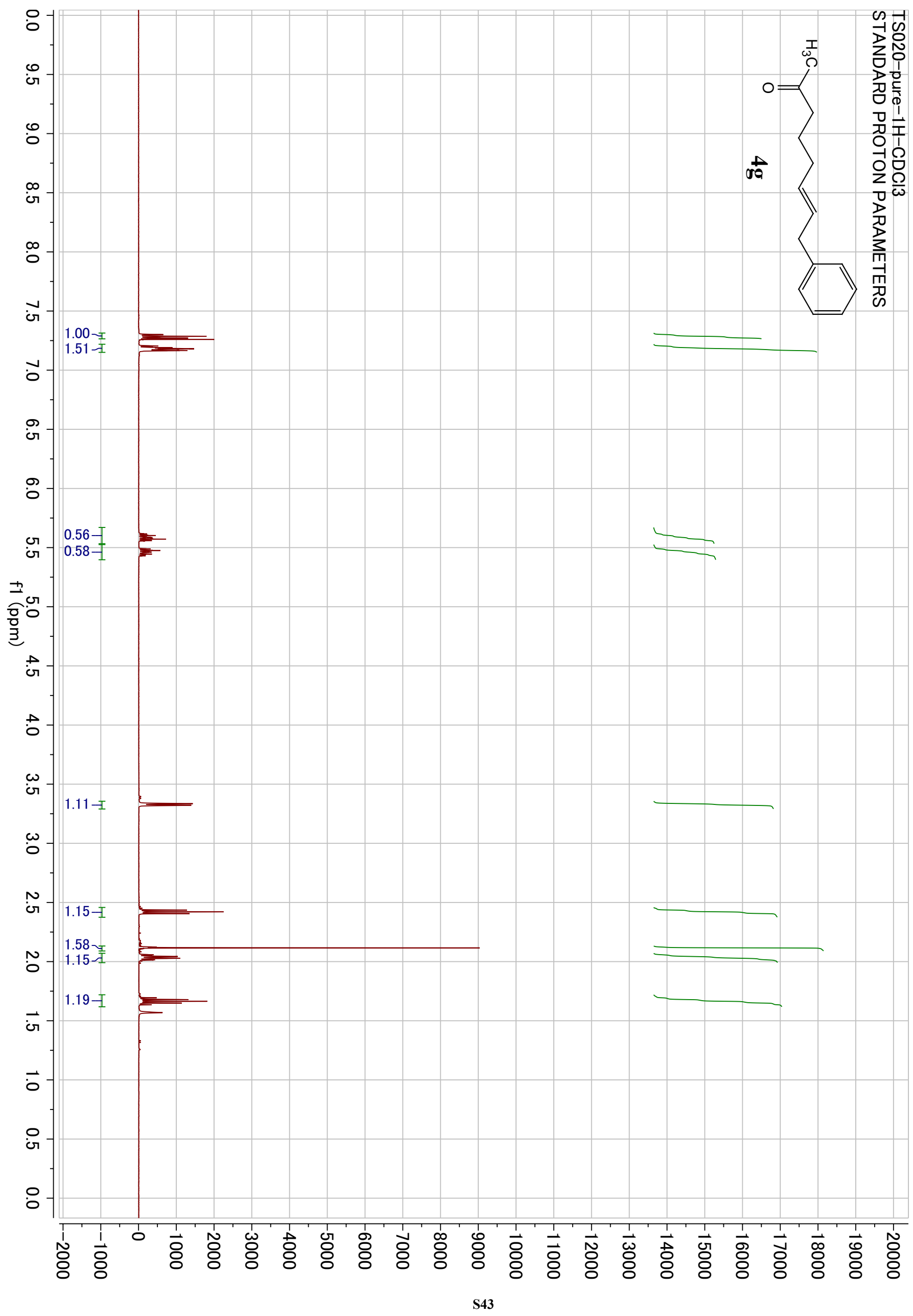




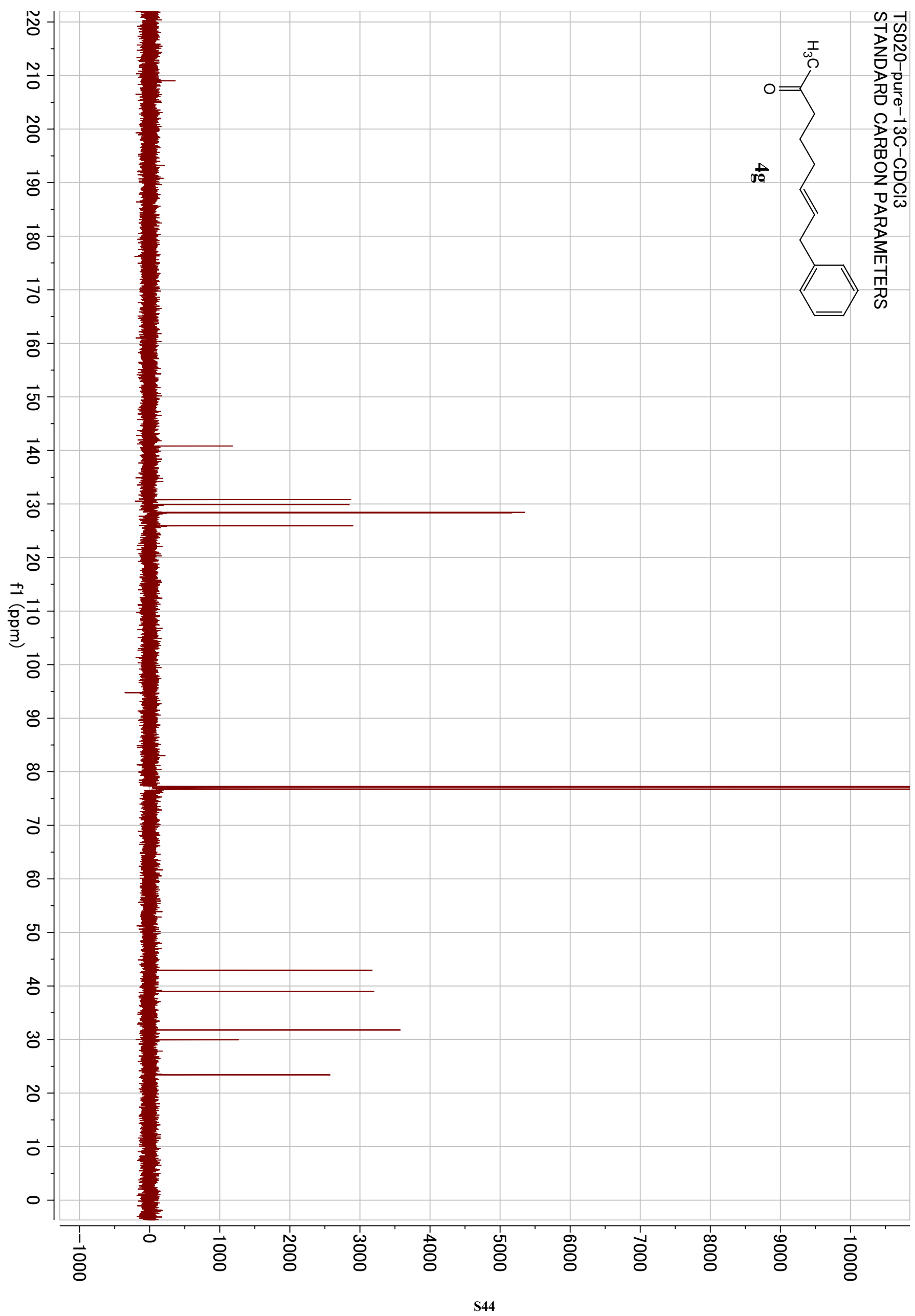




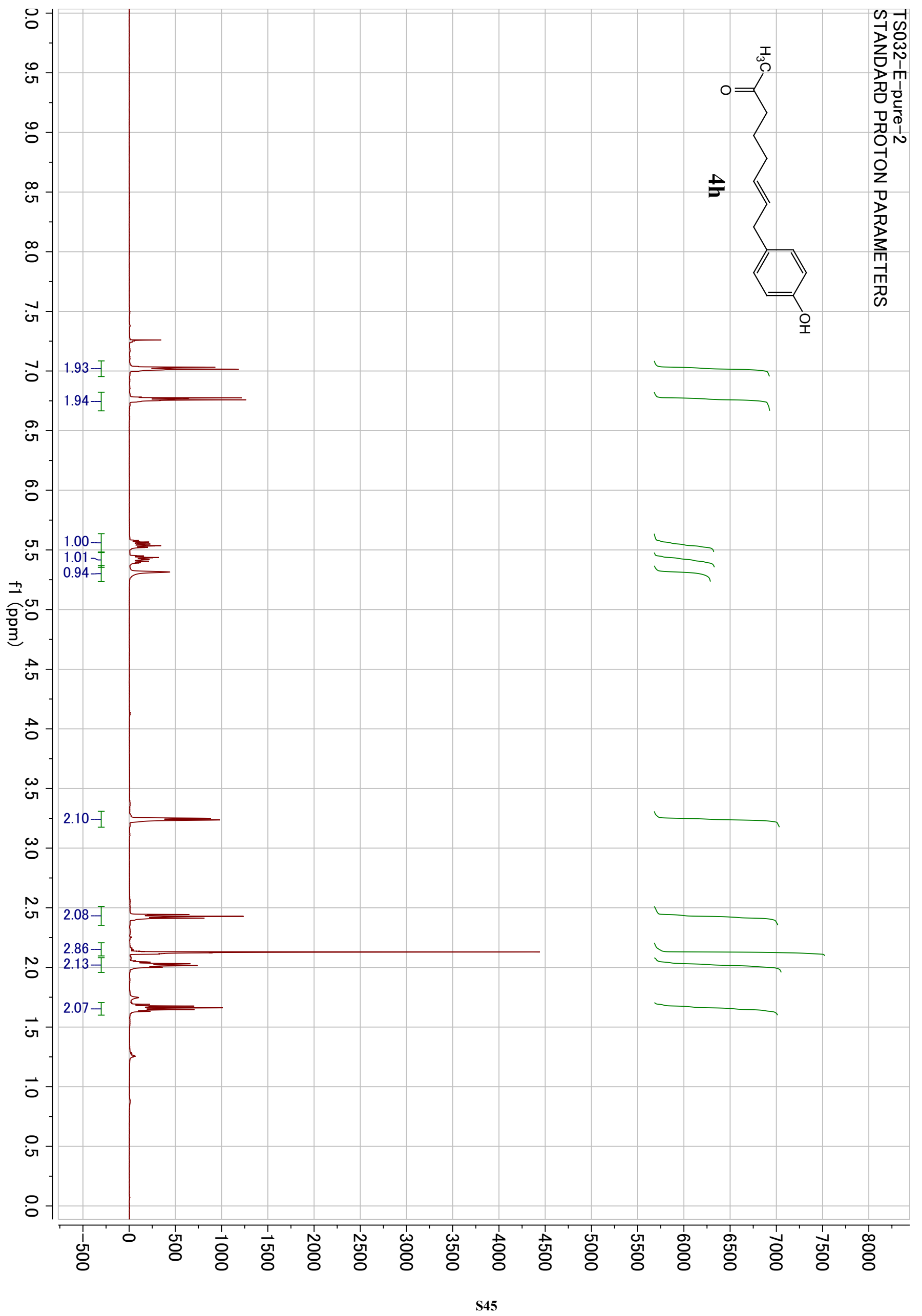




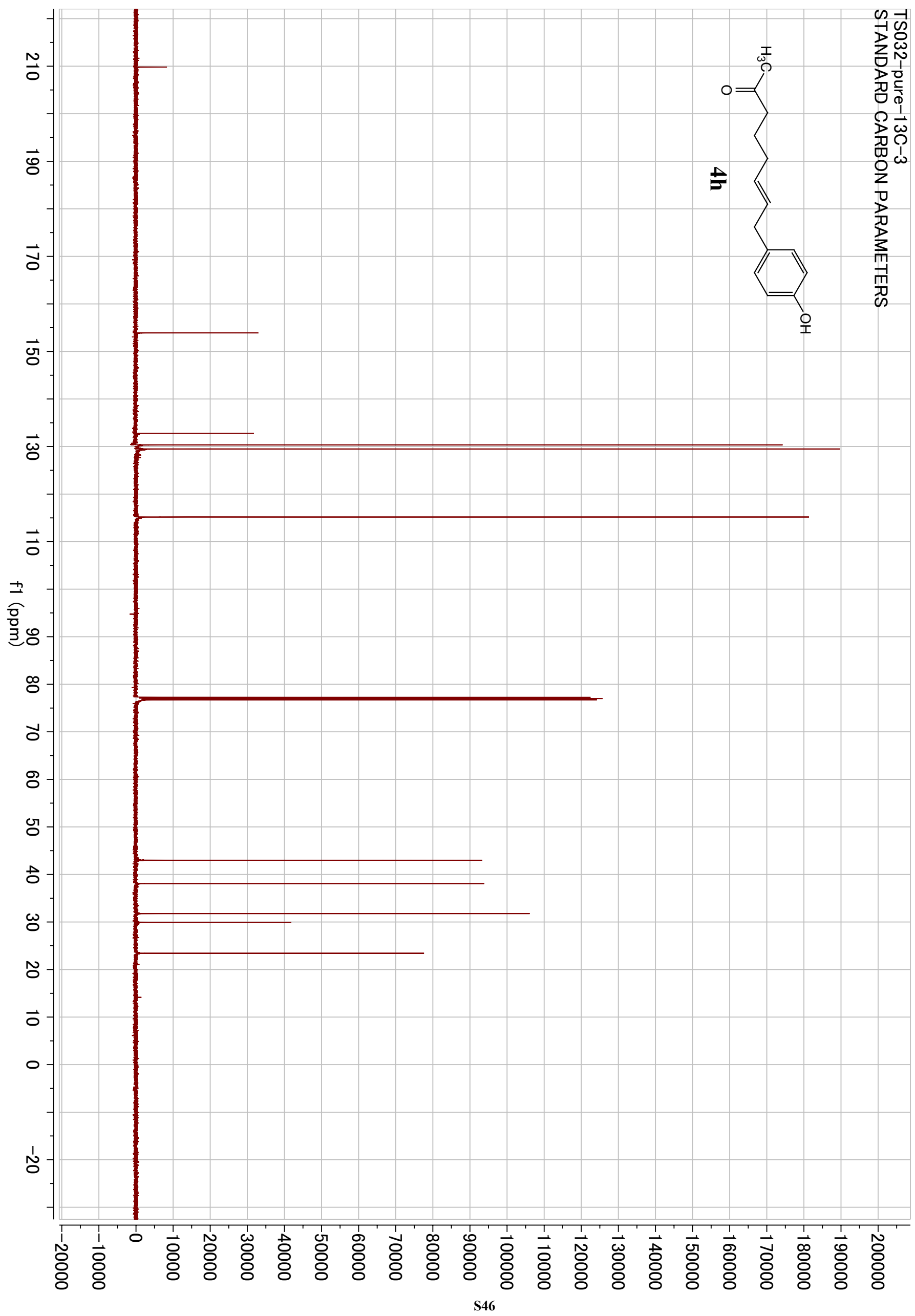




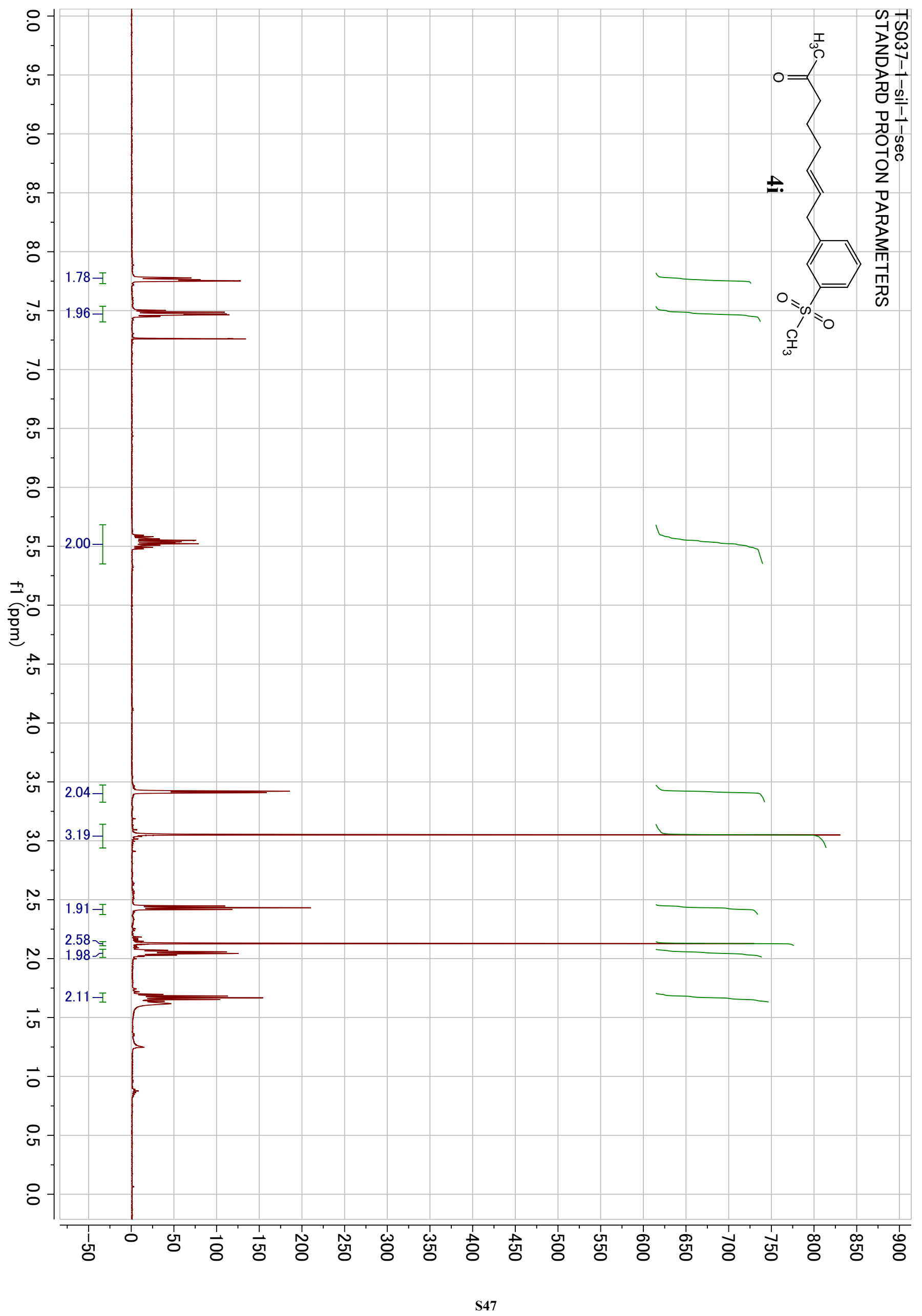




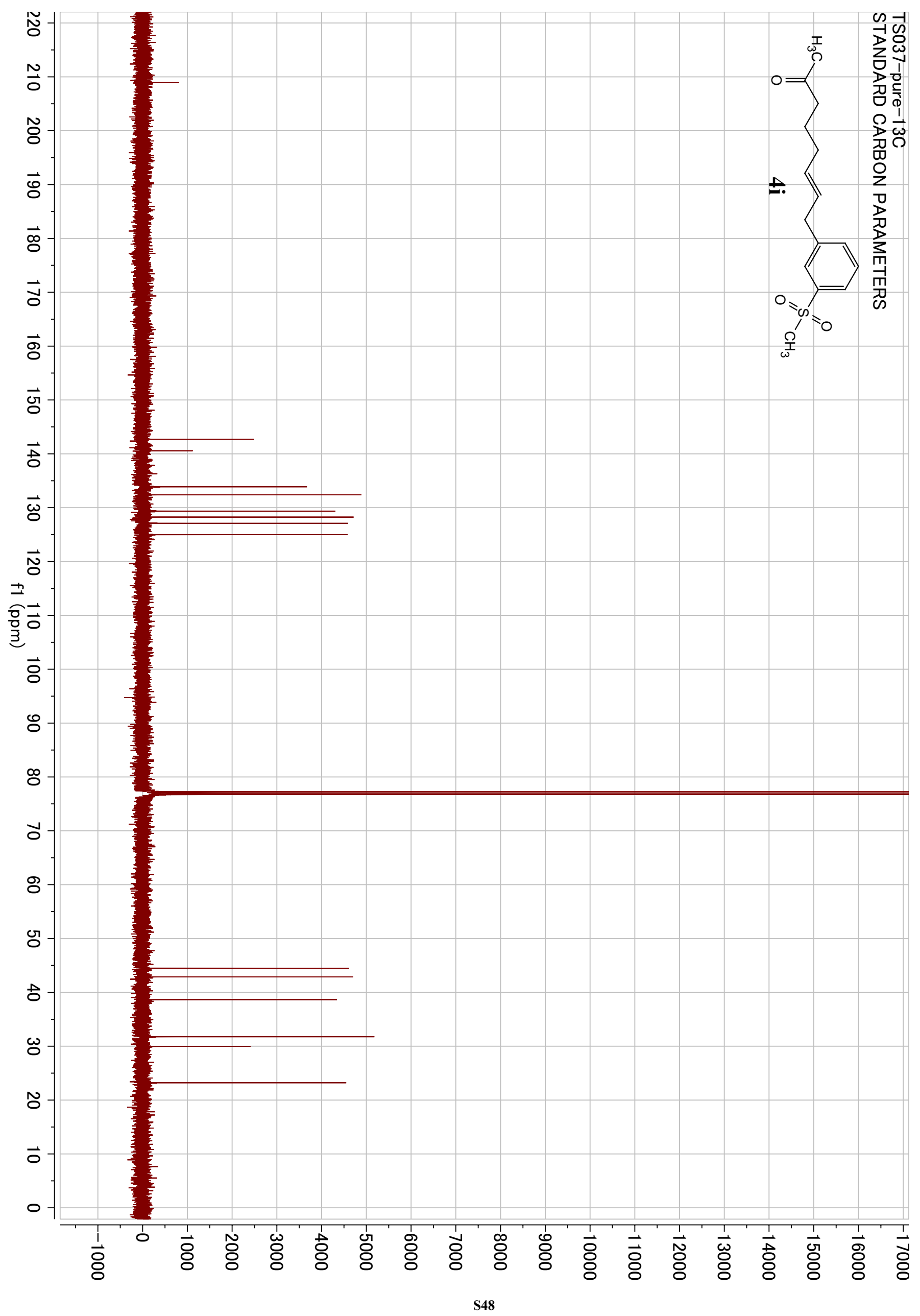




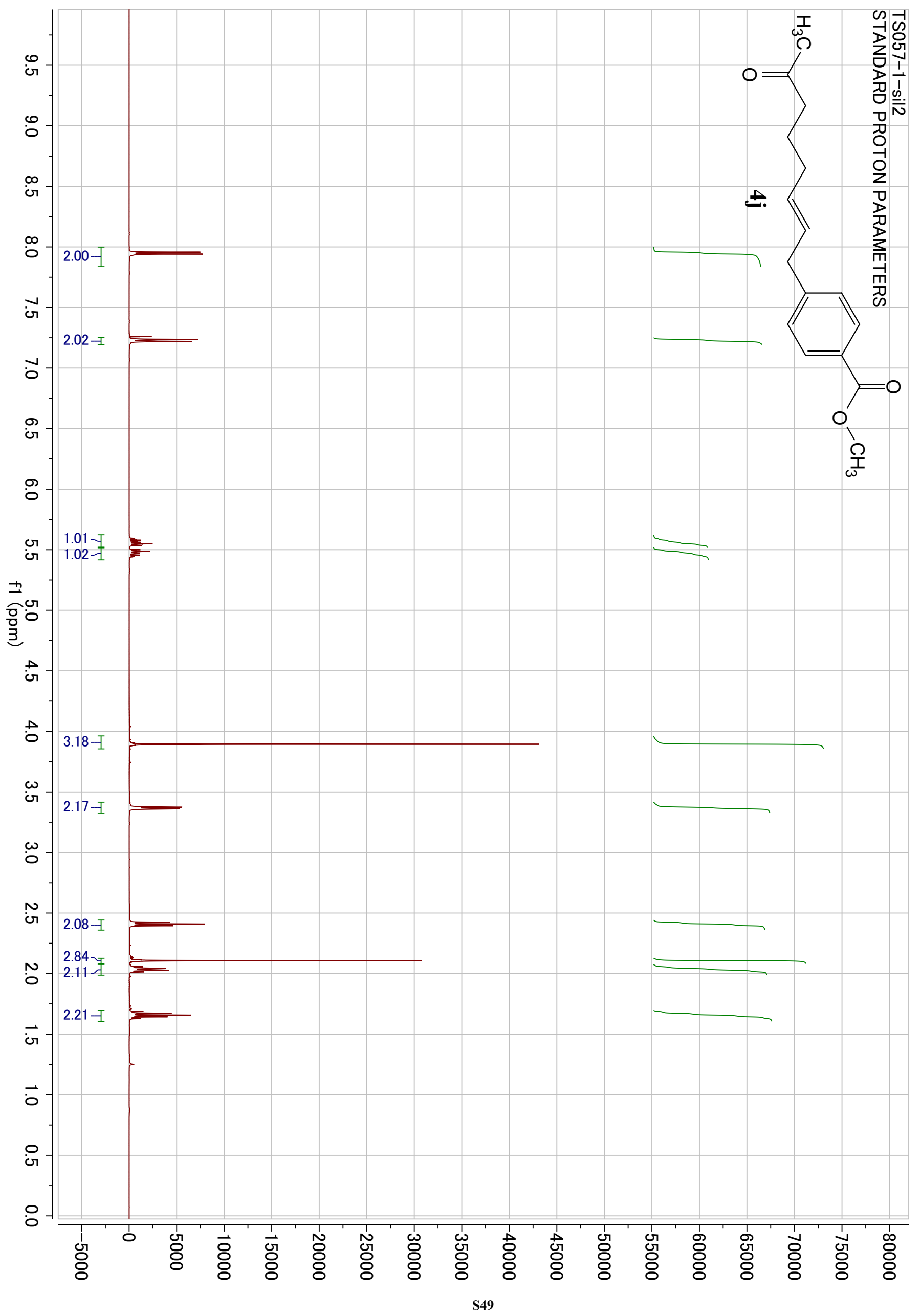




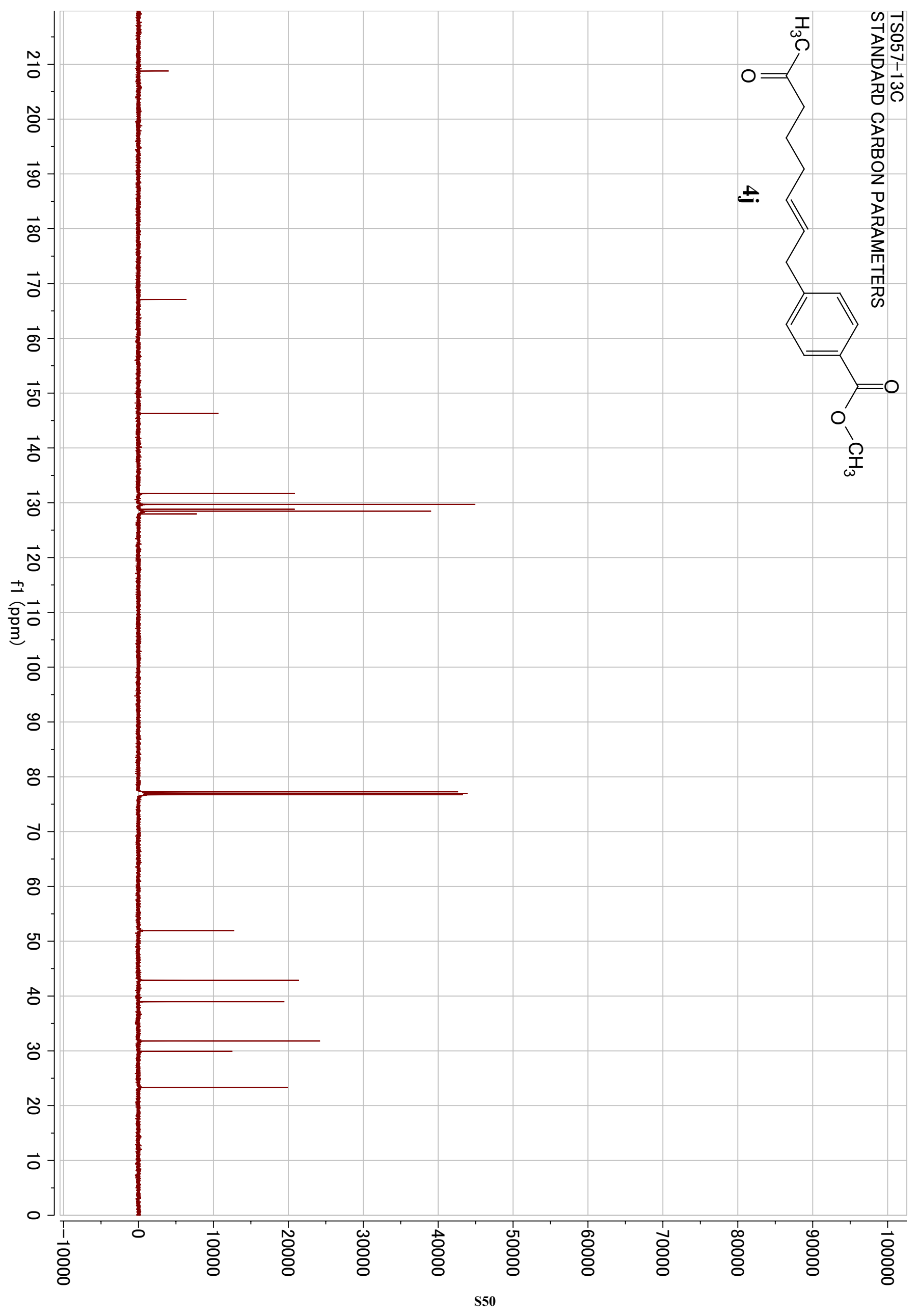




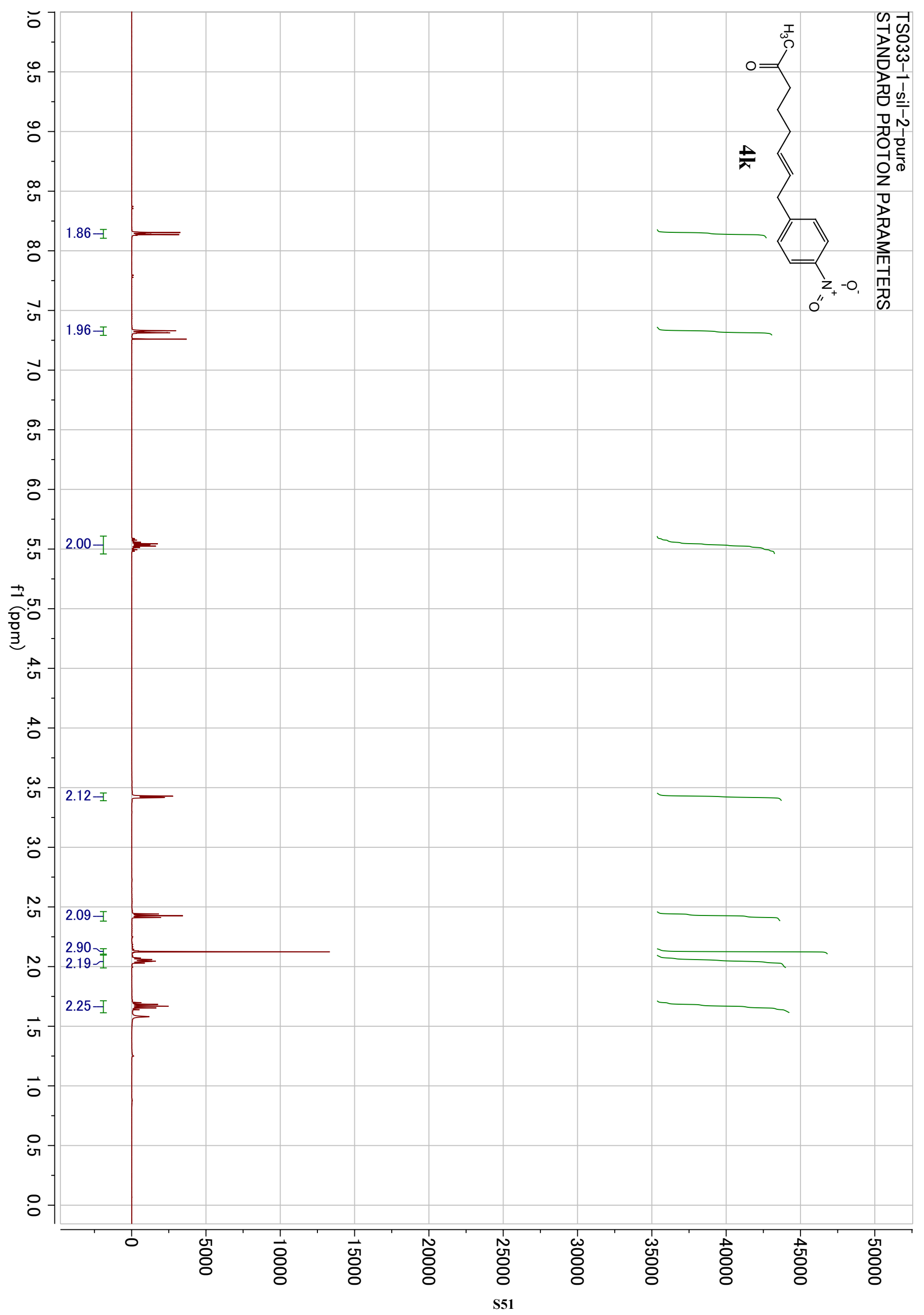




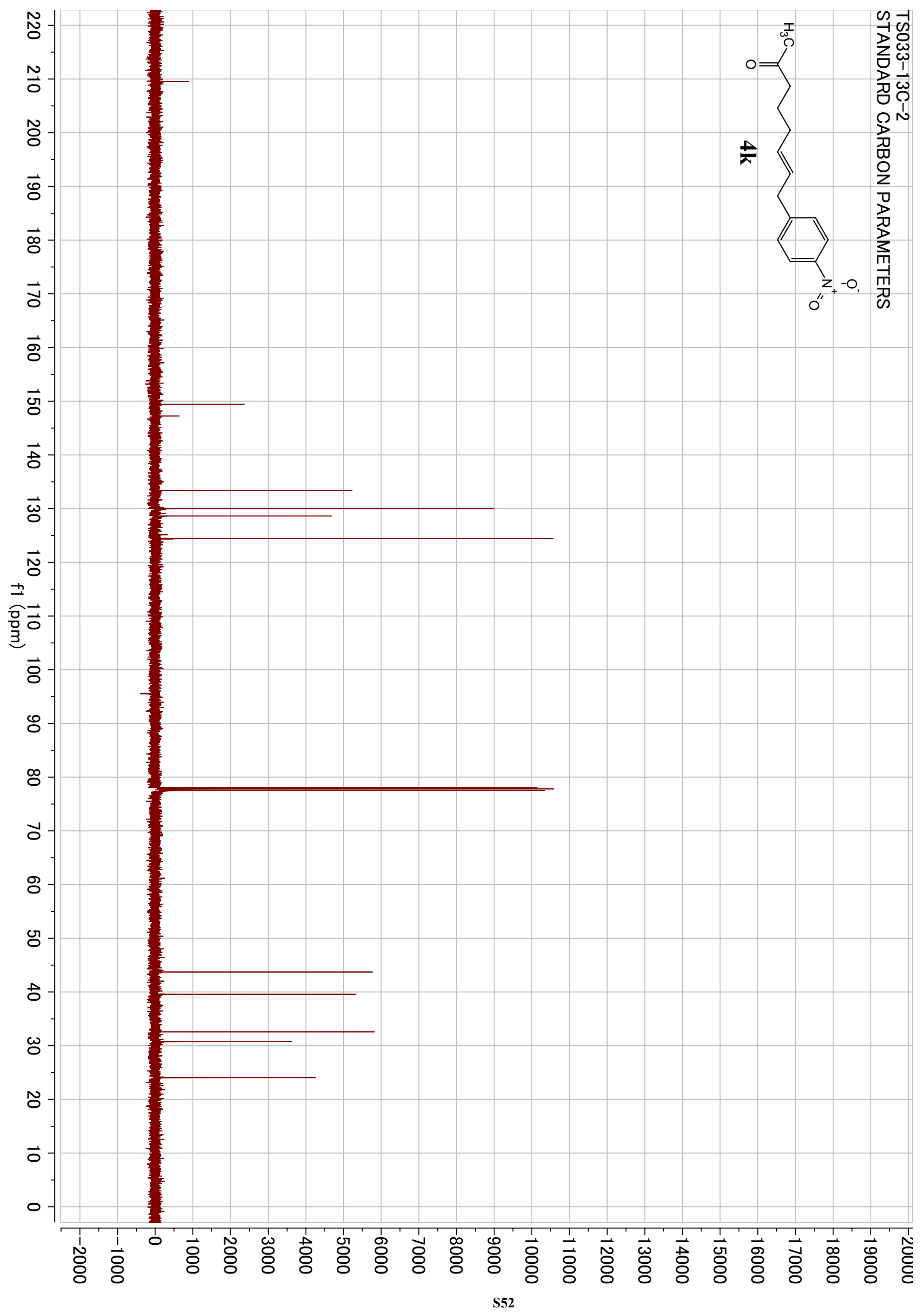




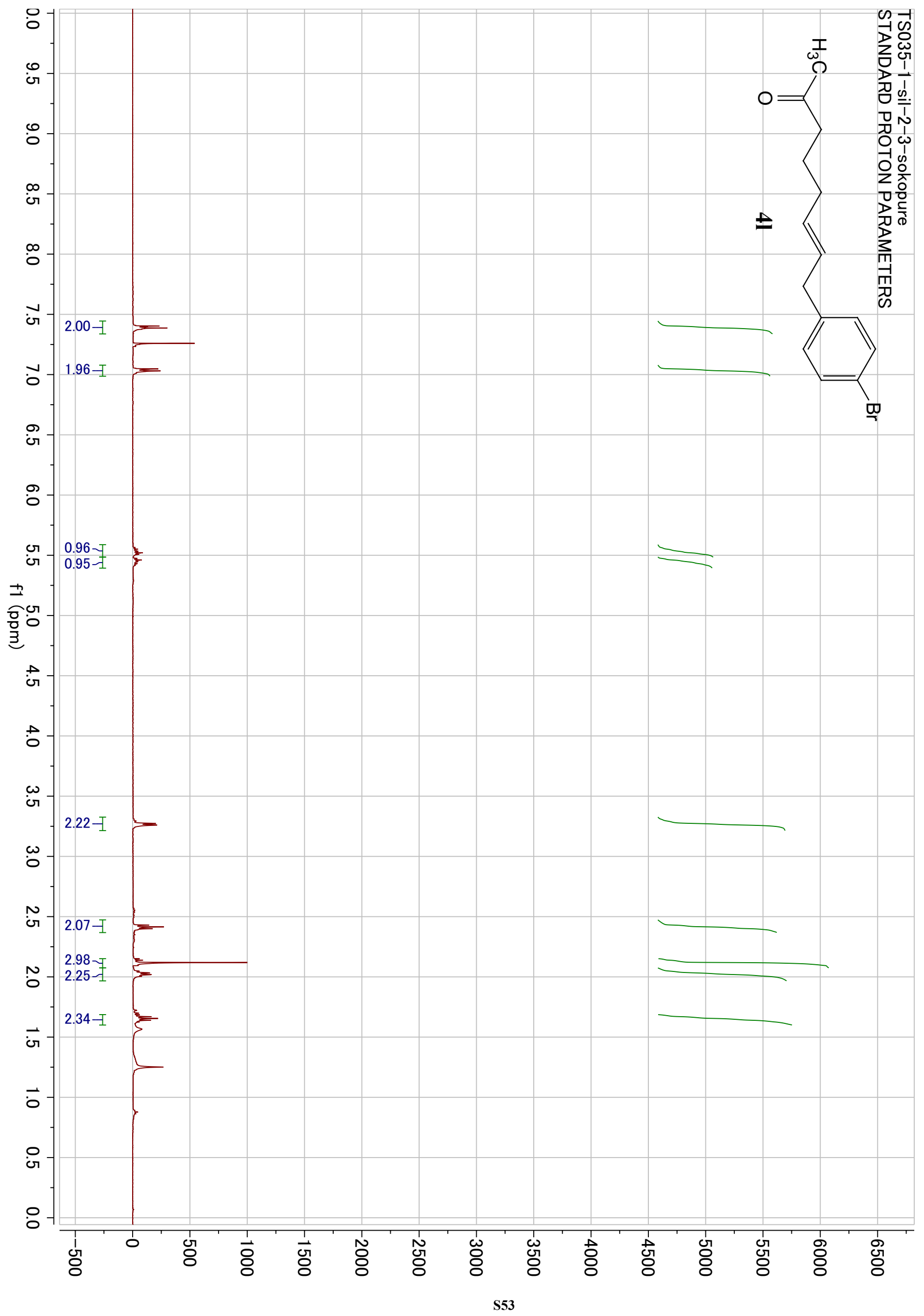




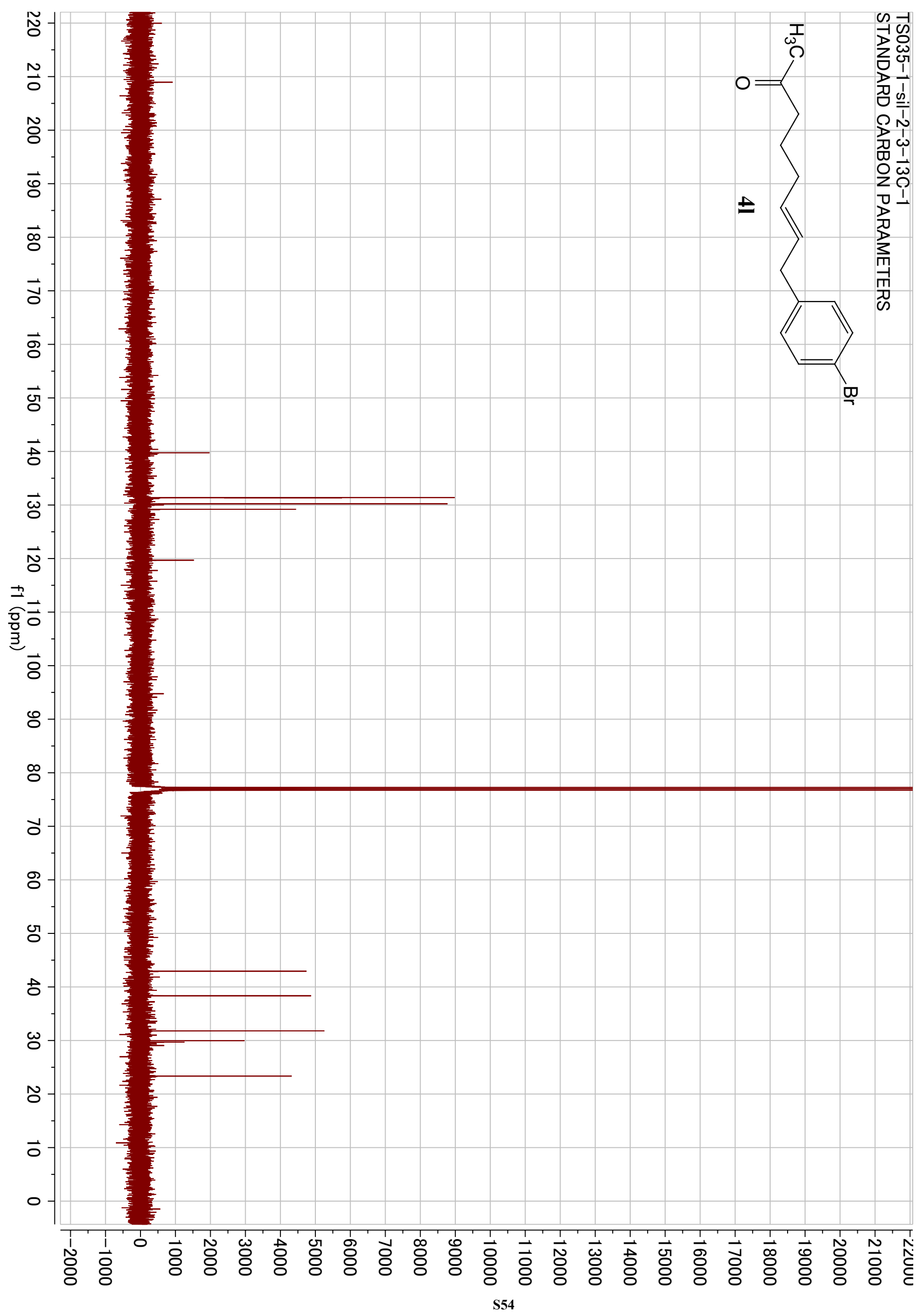




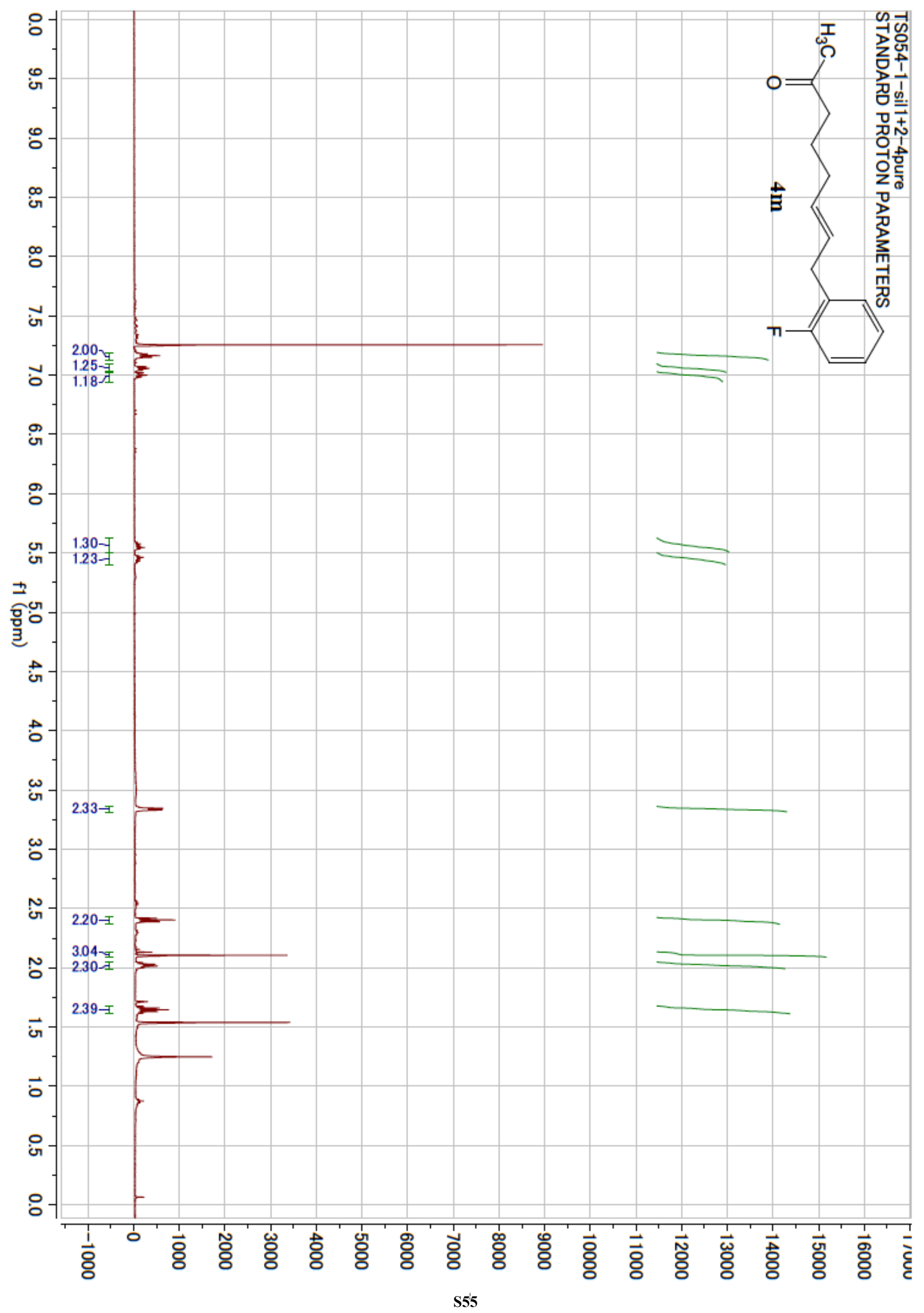




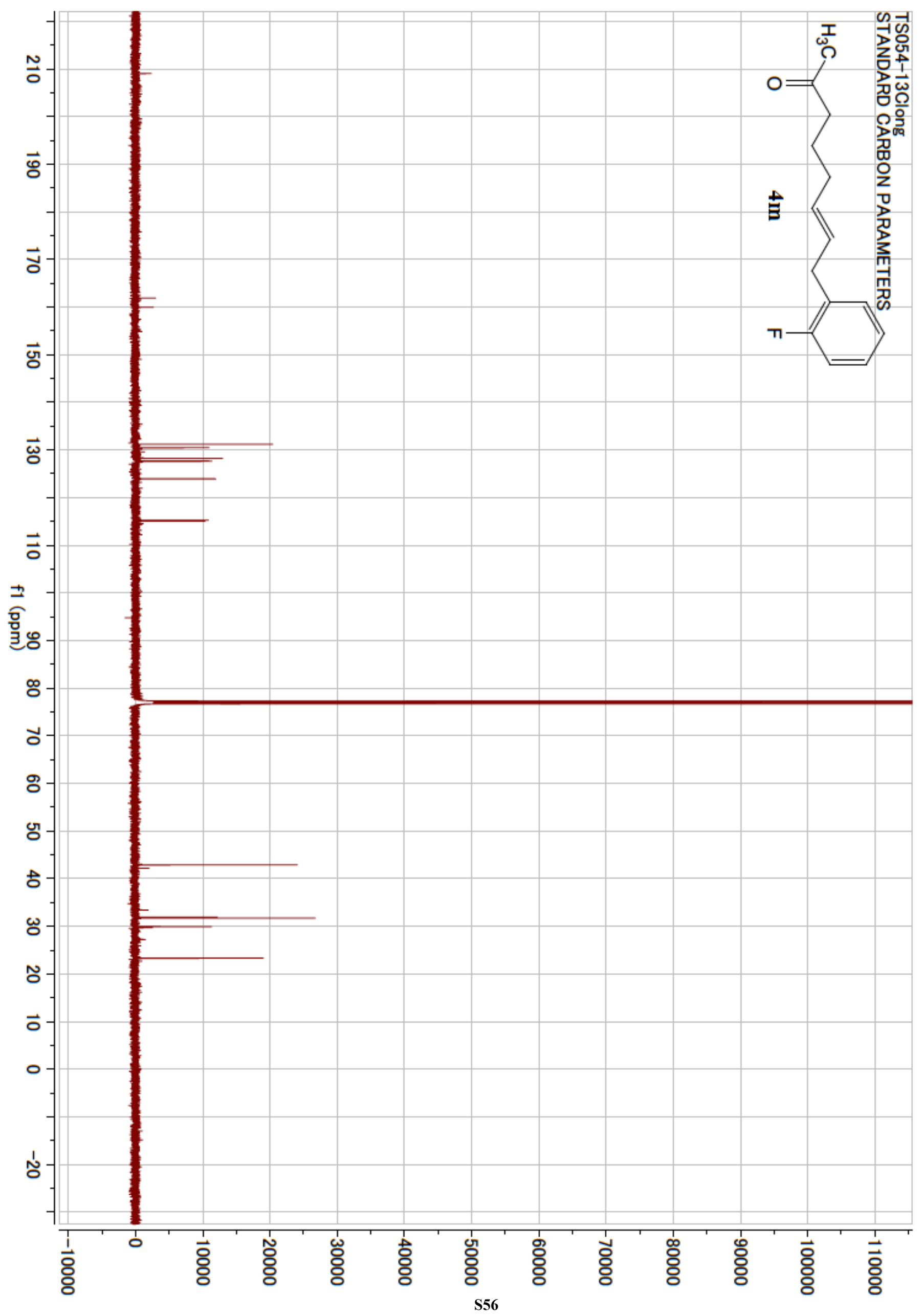



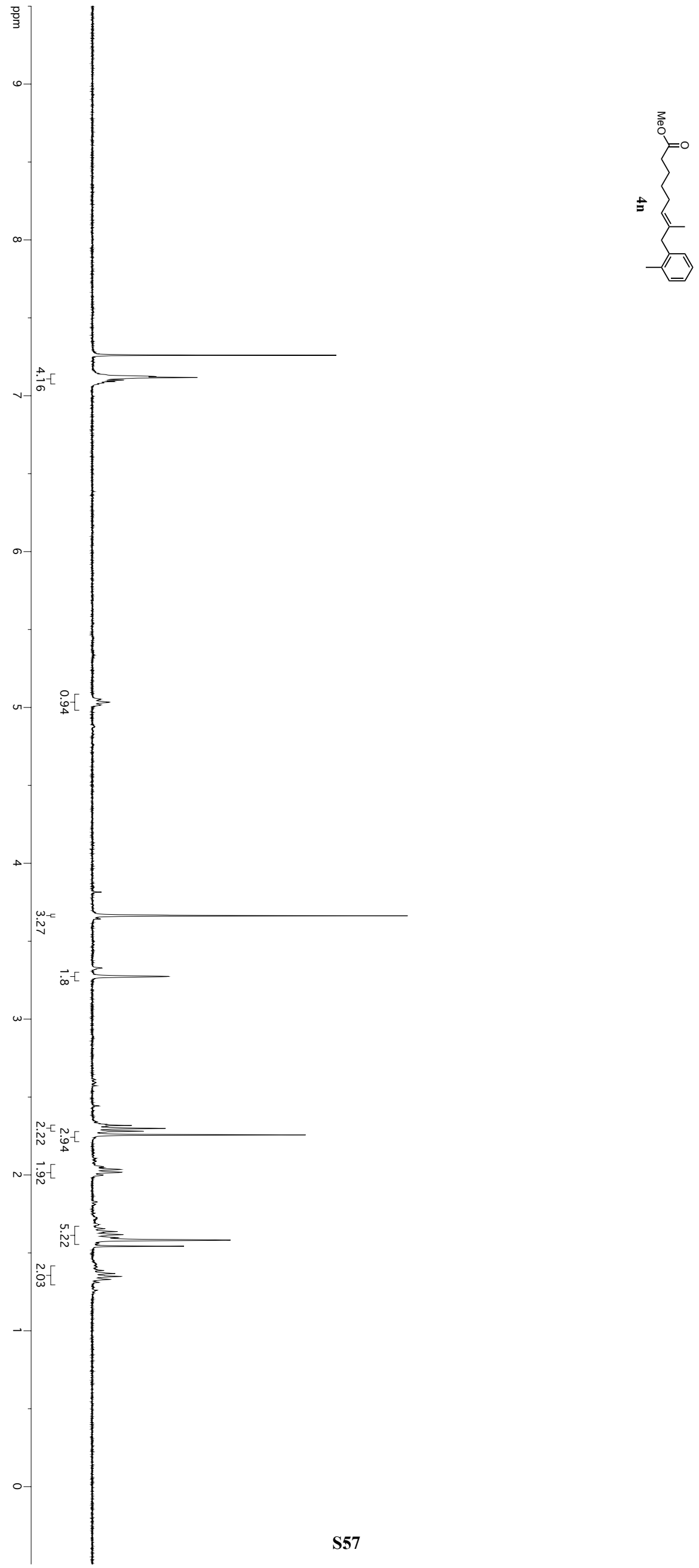

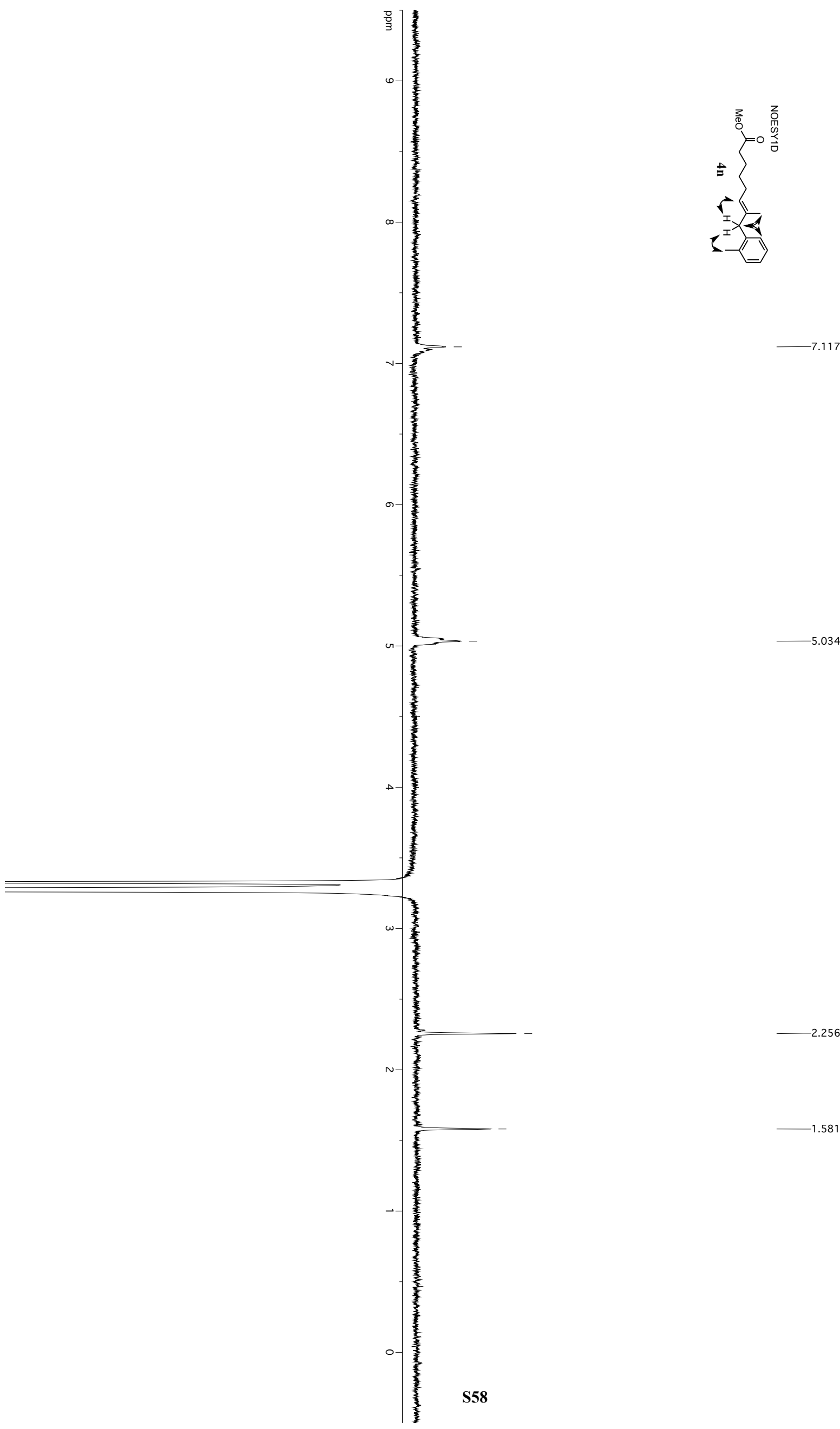

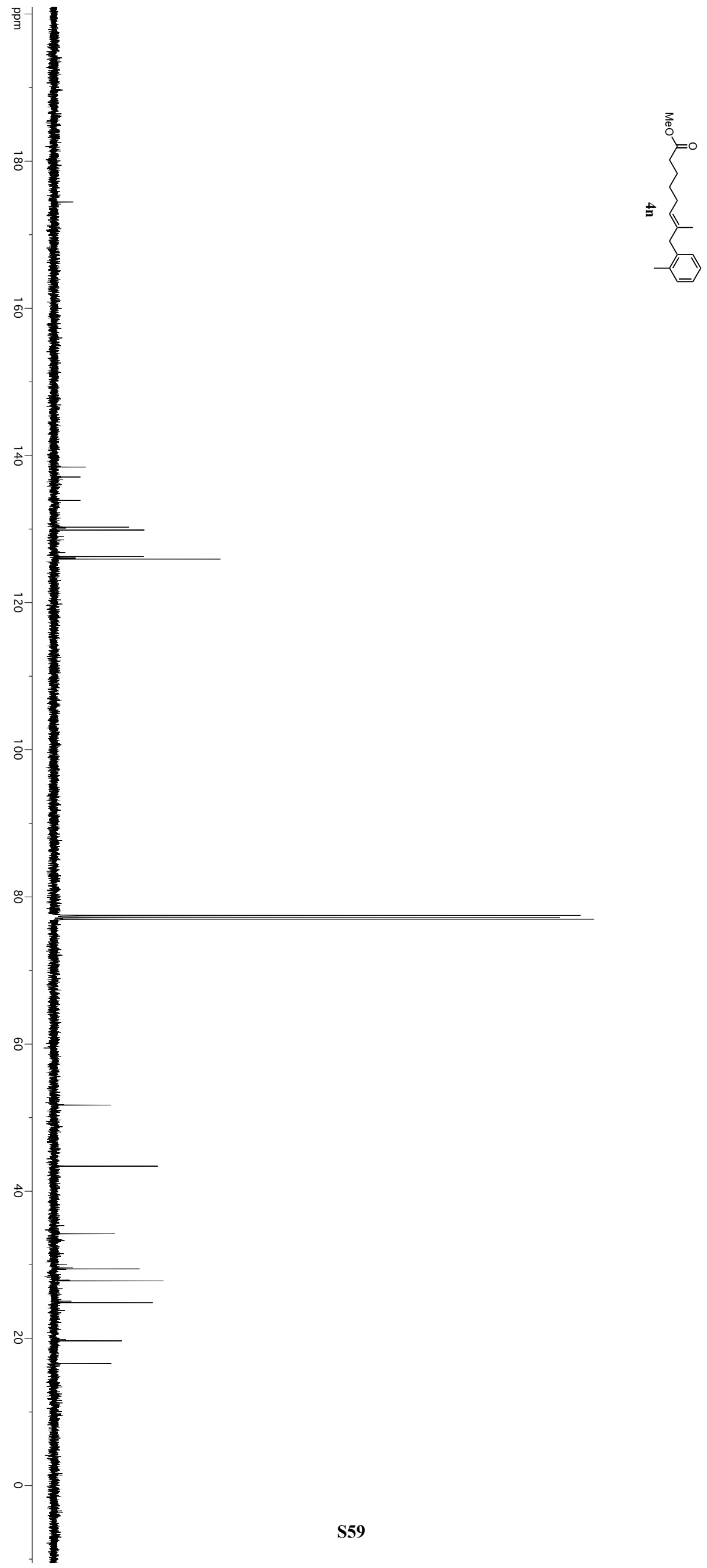


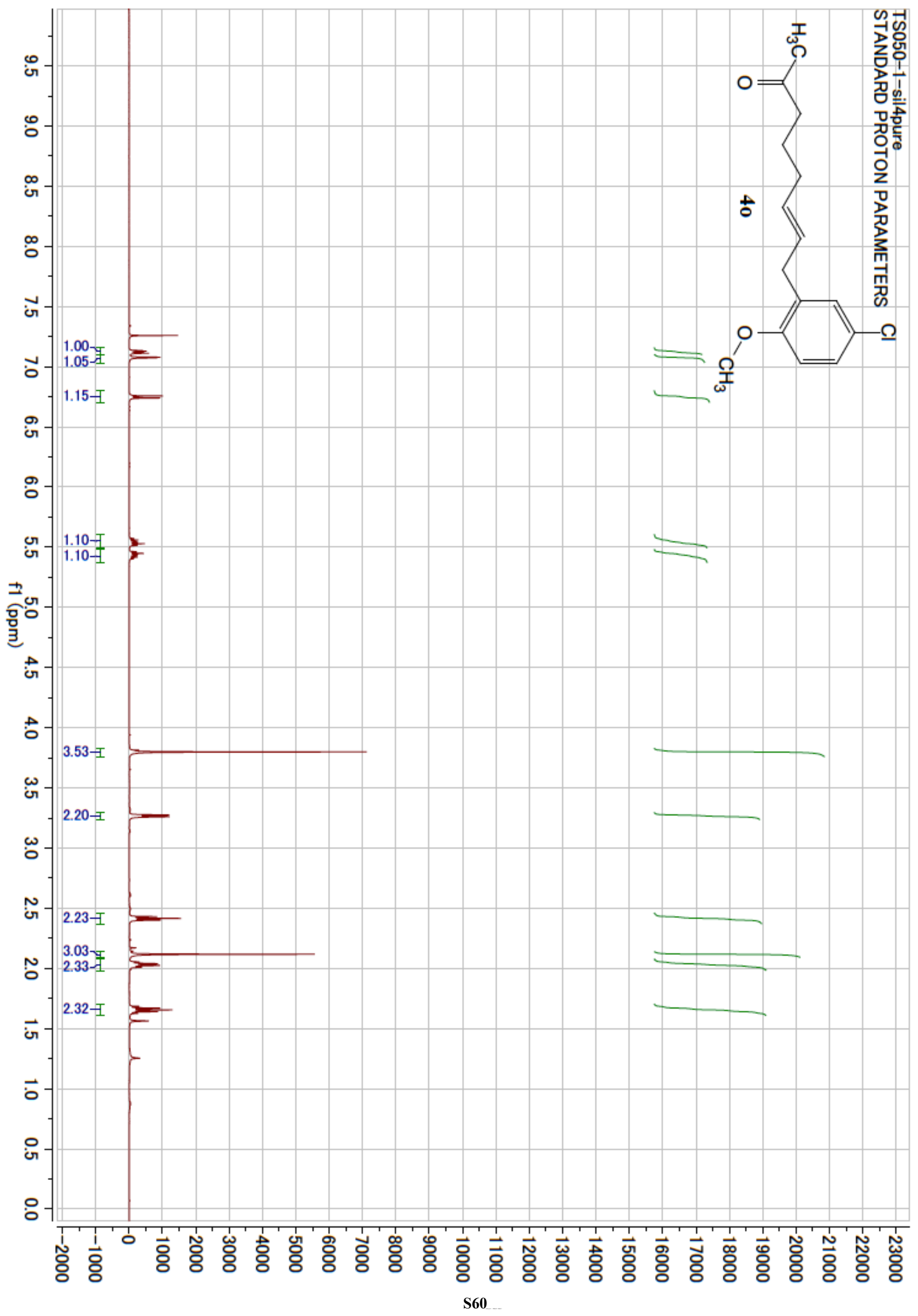




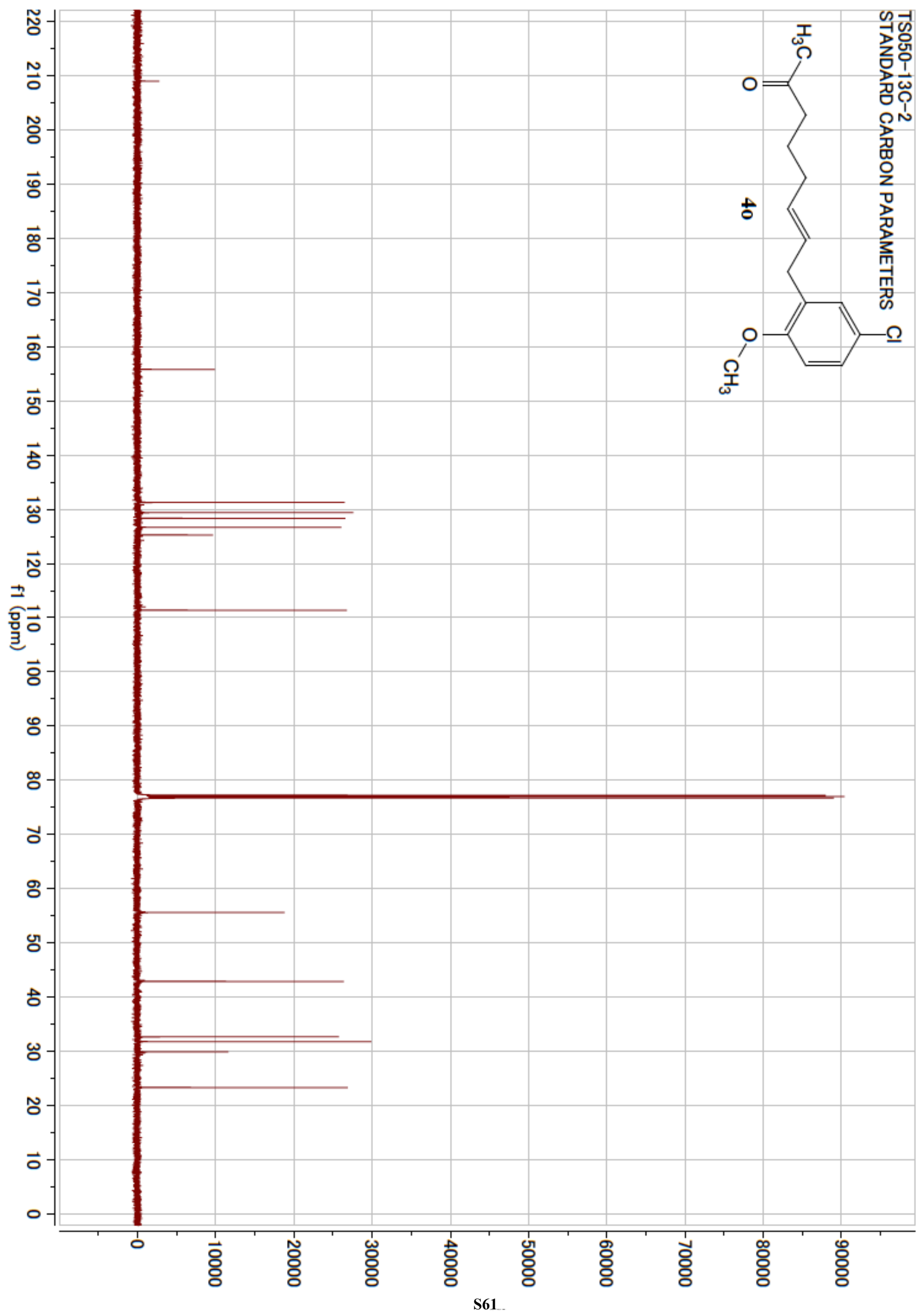




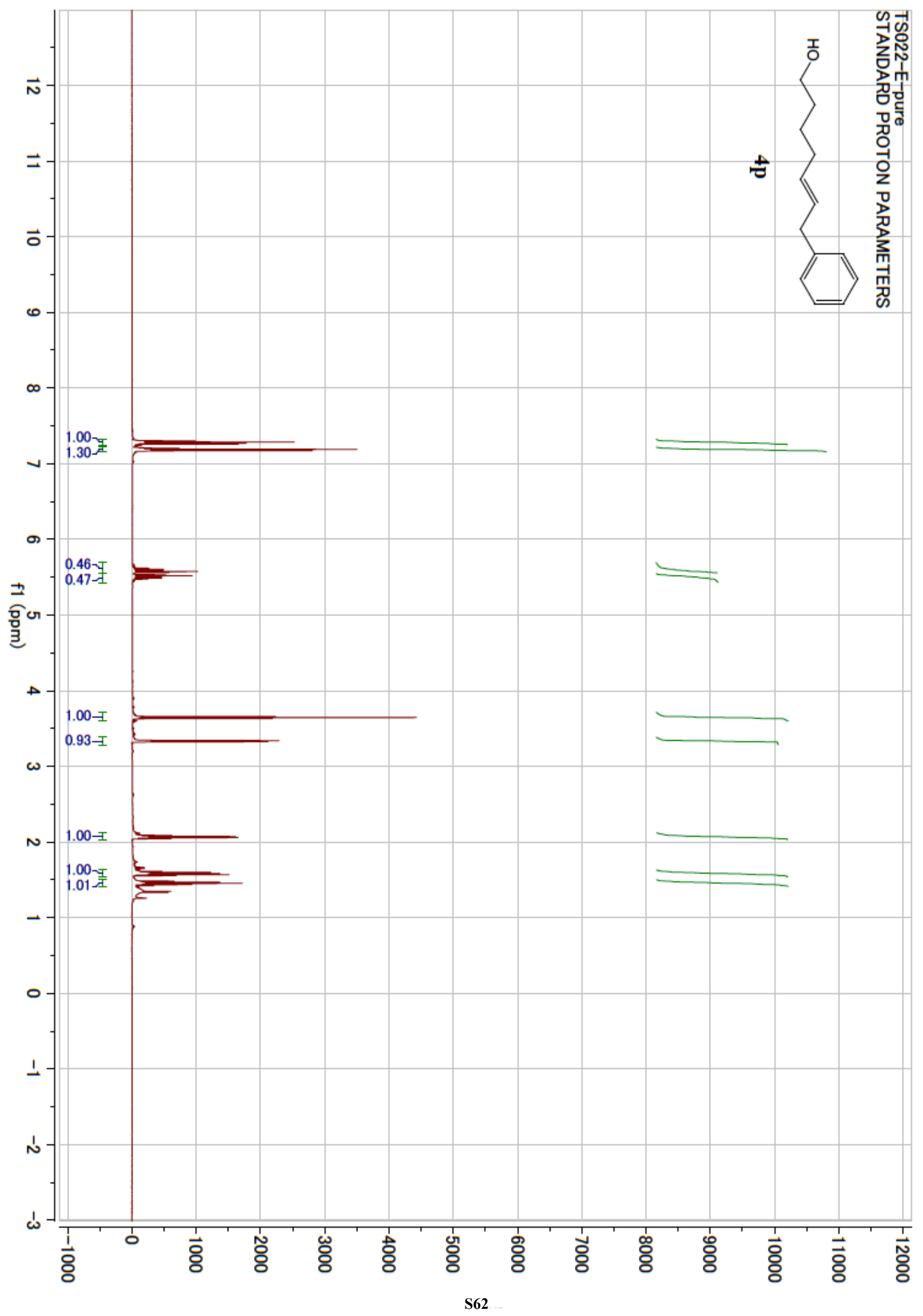




$$
E
$$




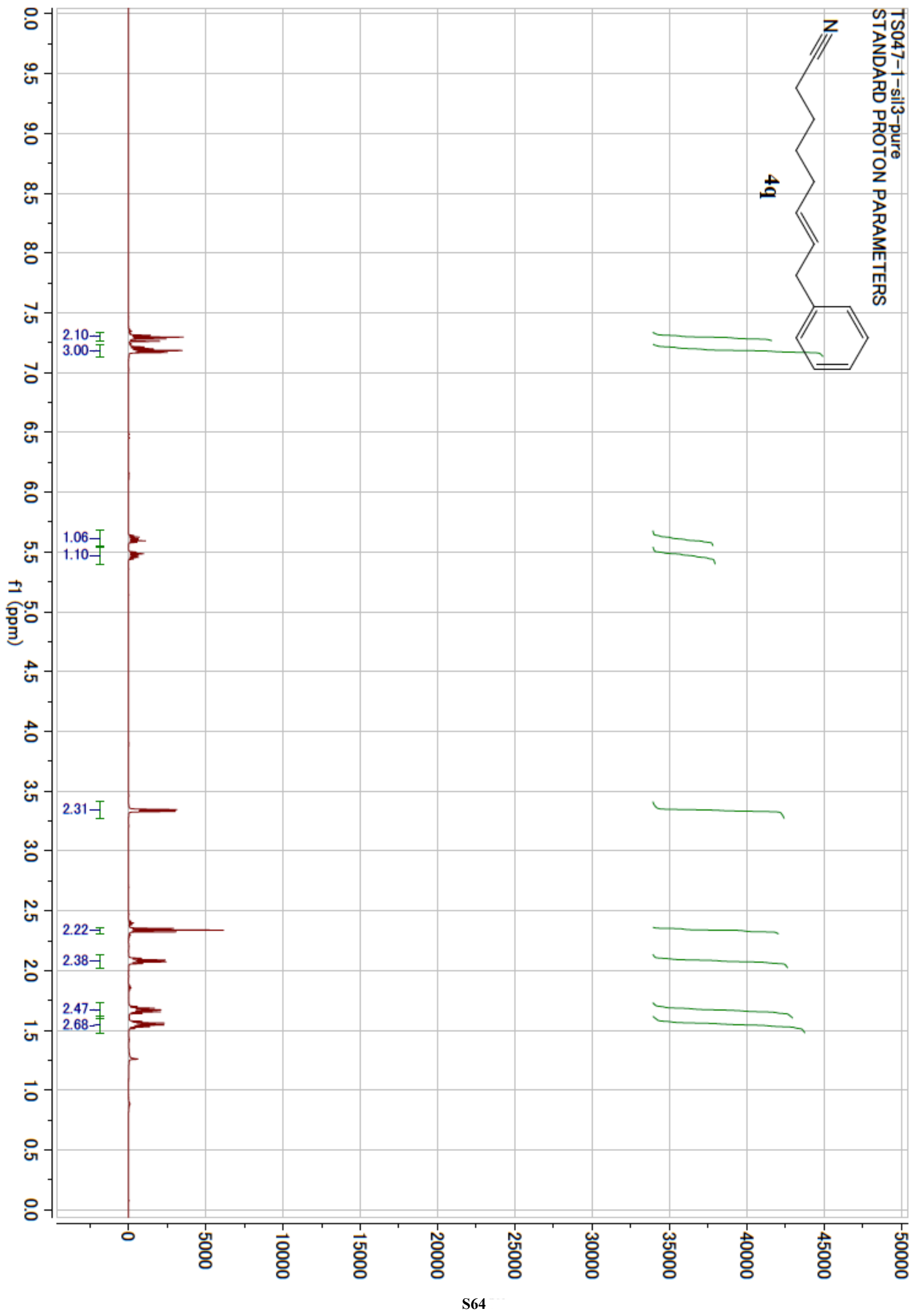




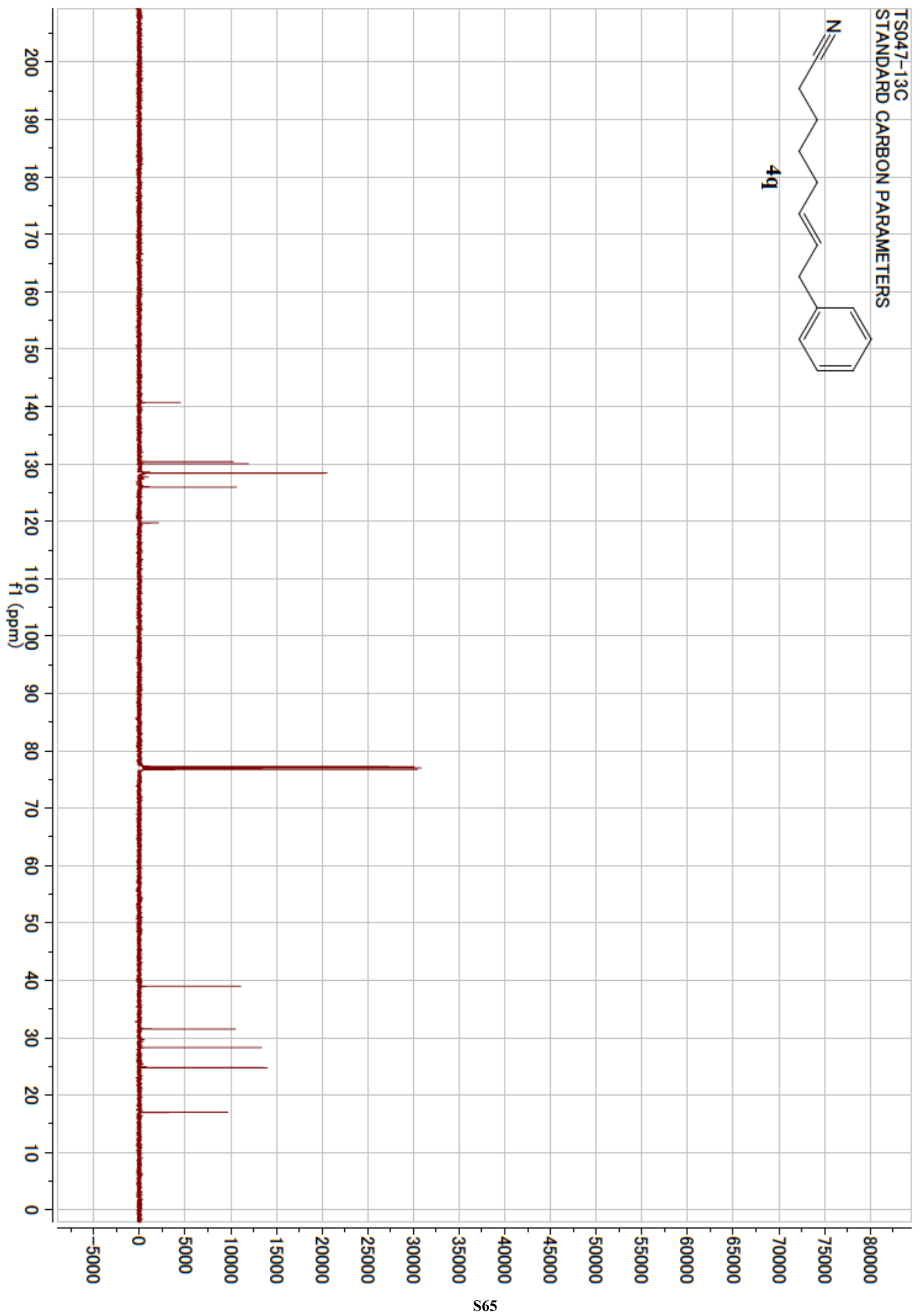




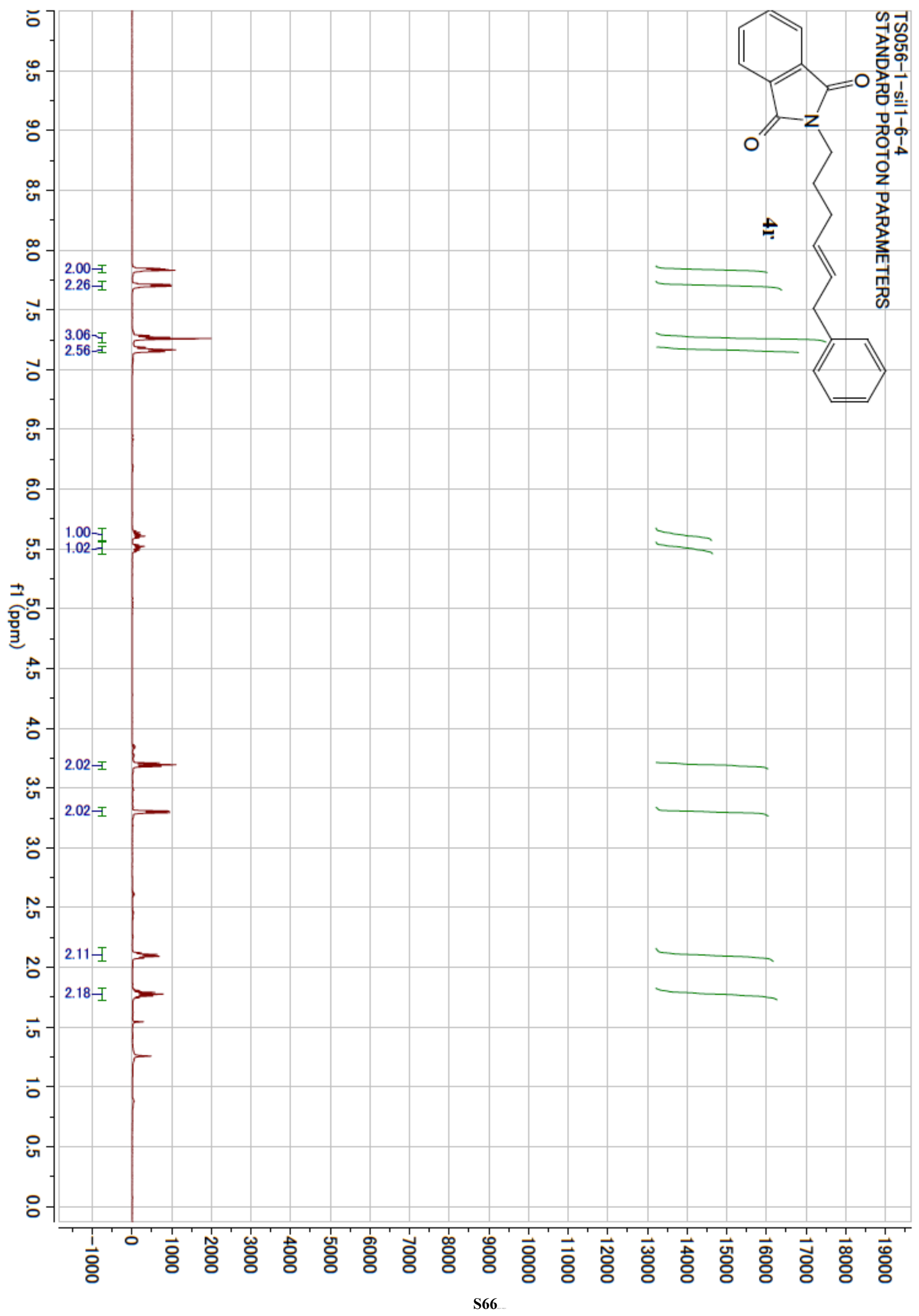




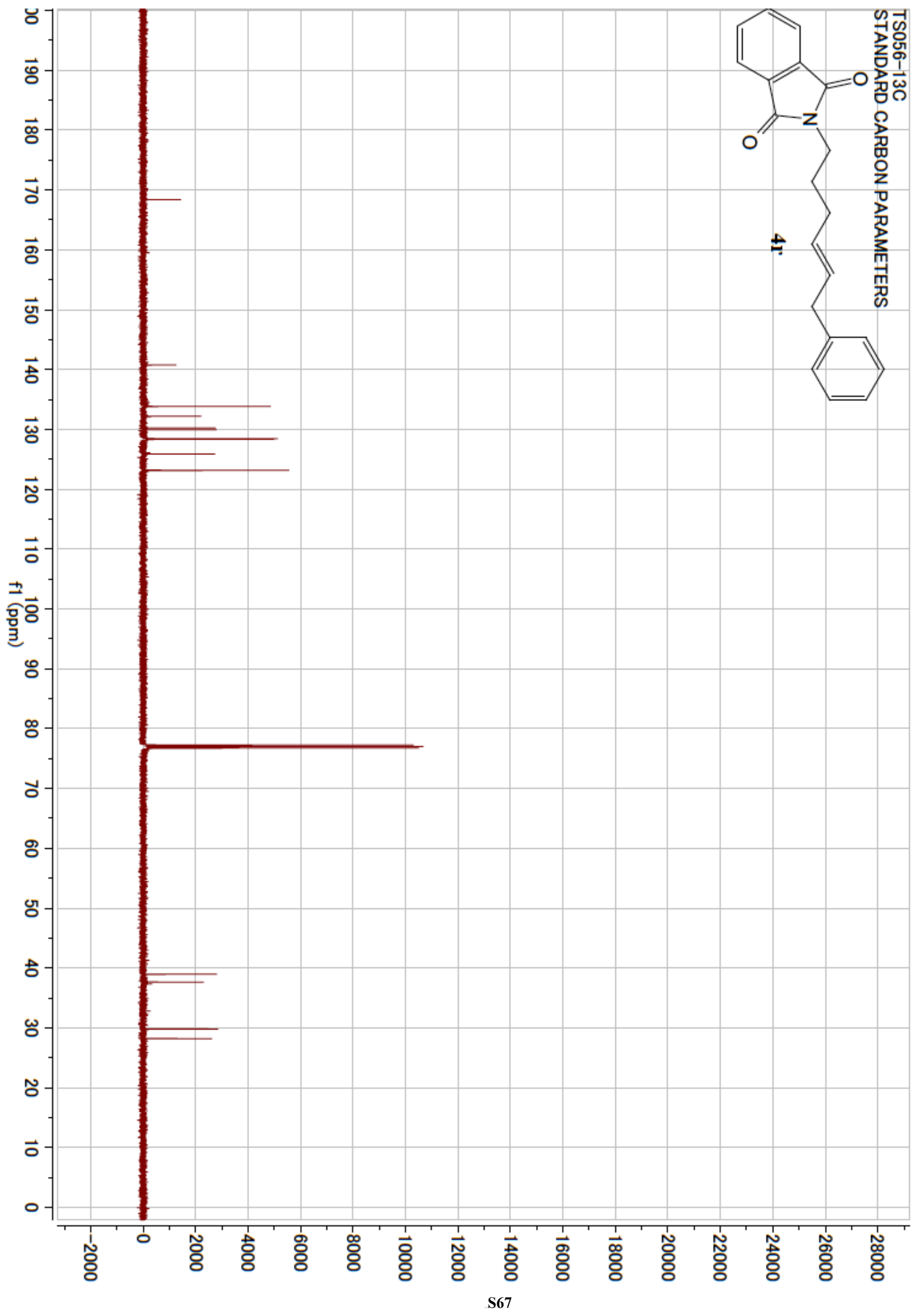




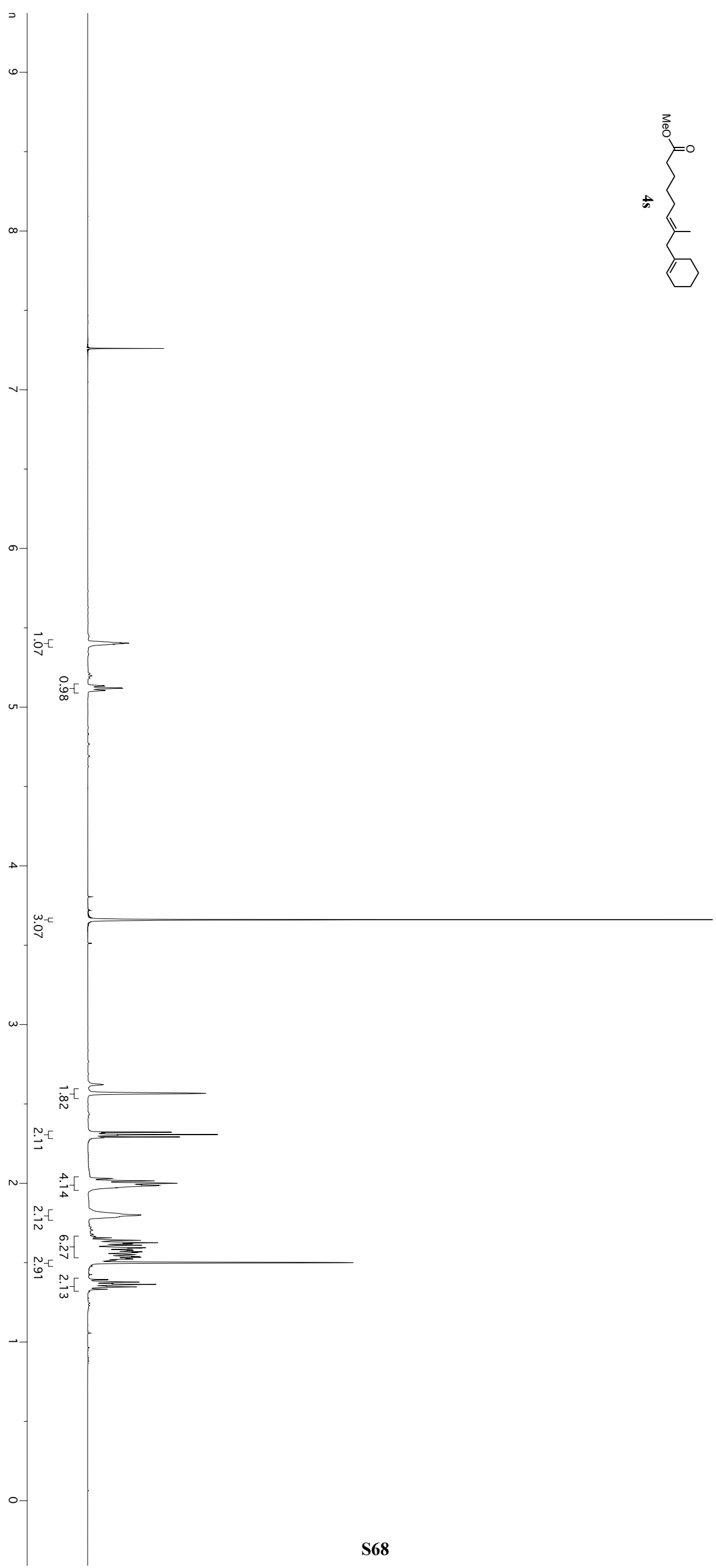




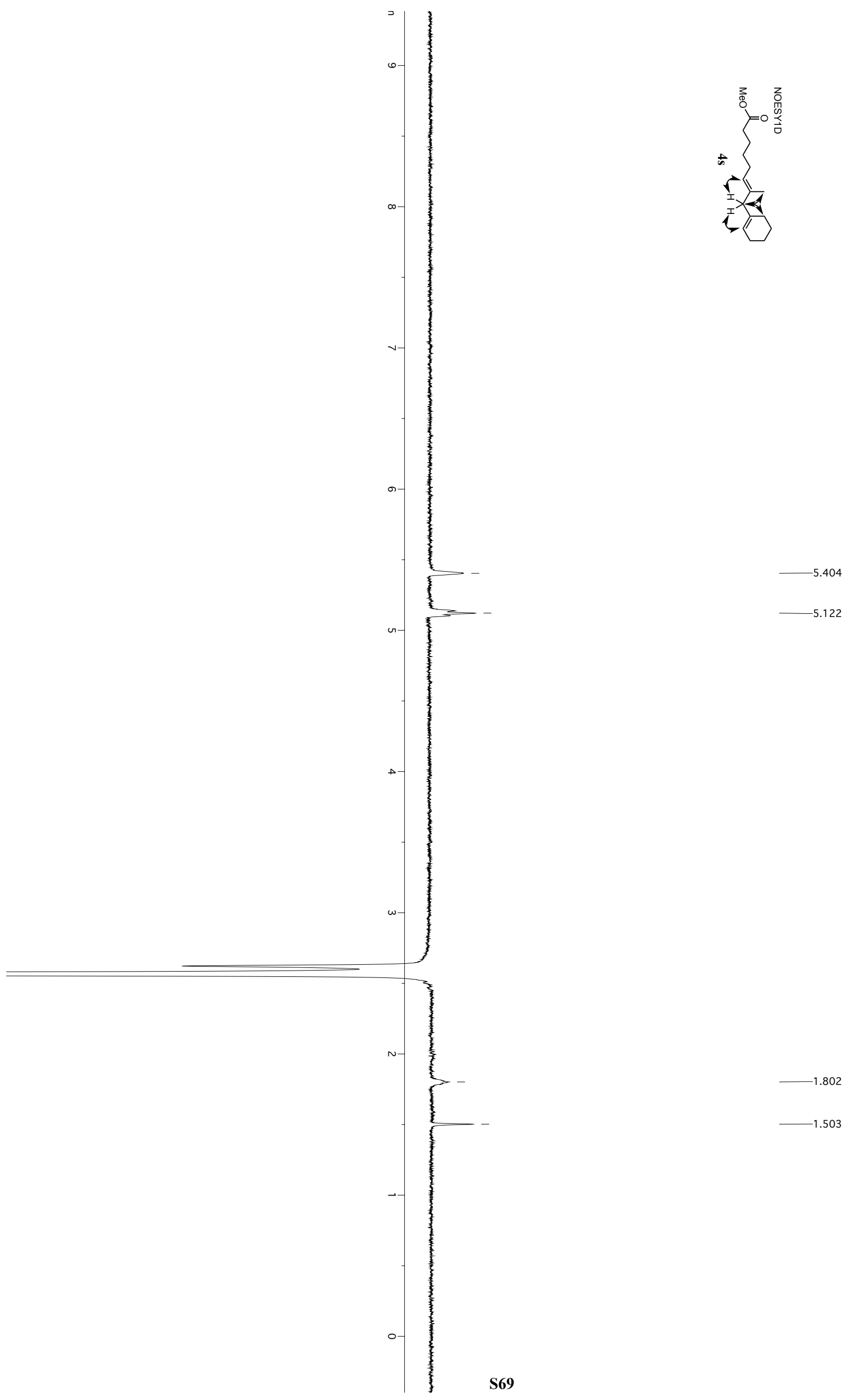



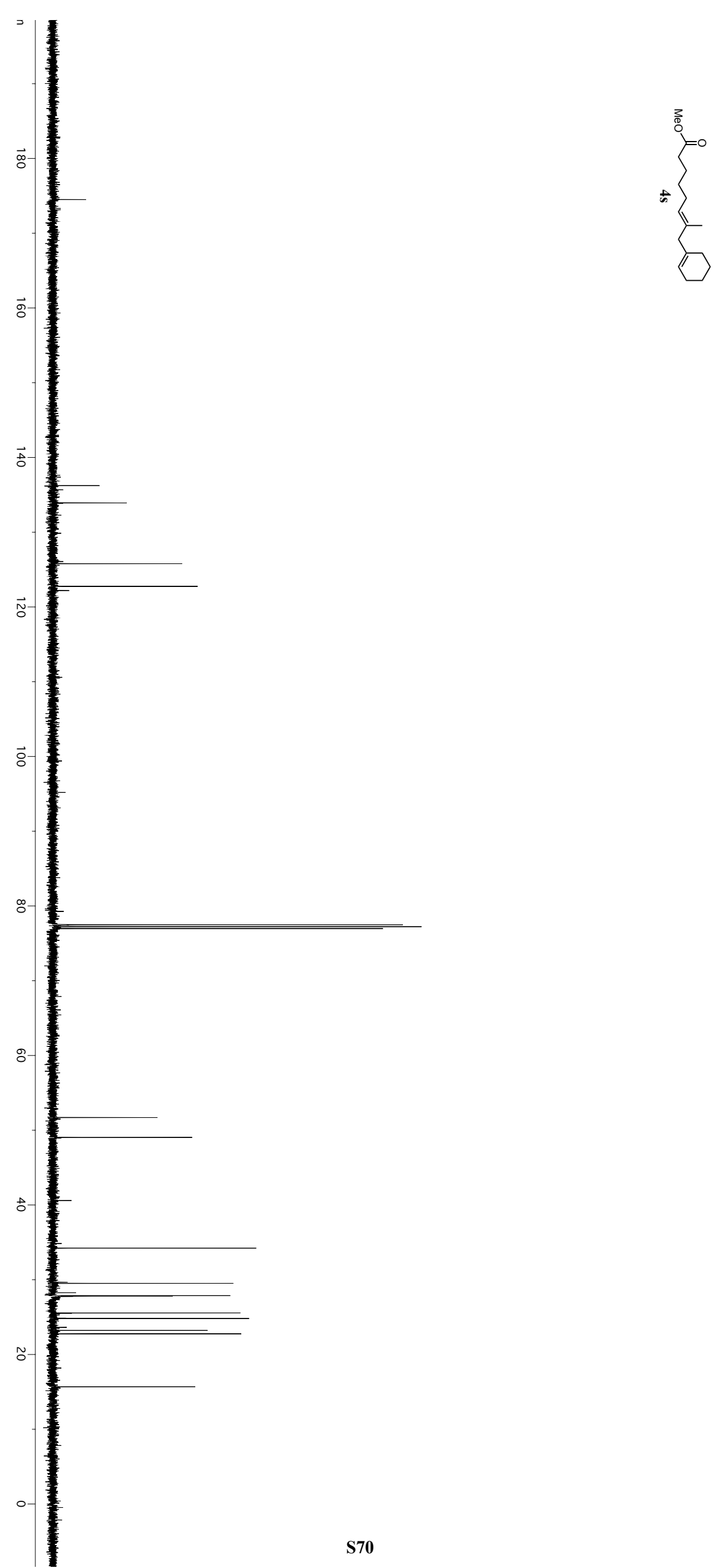


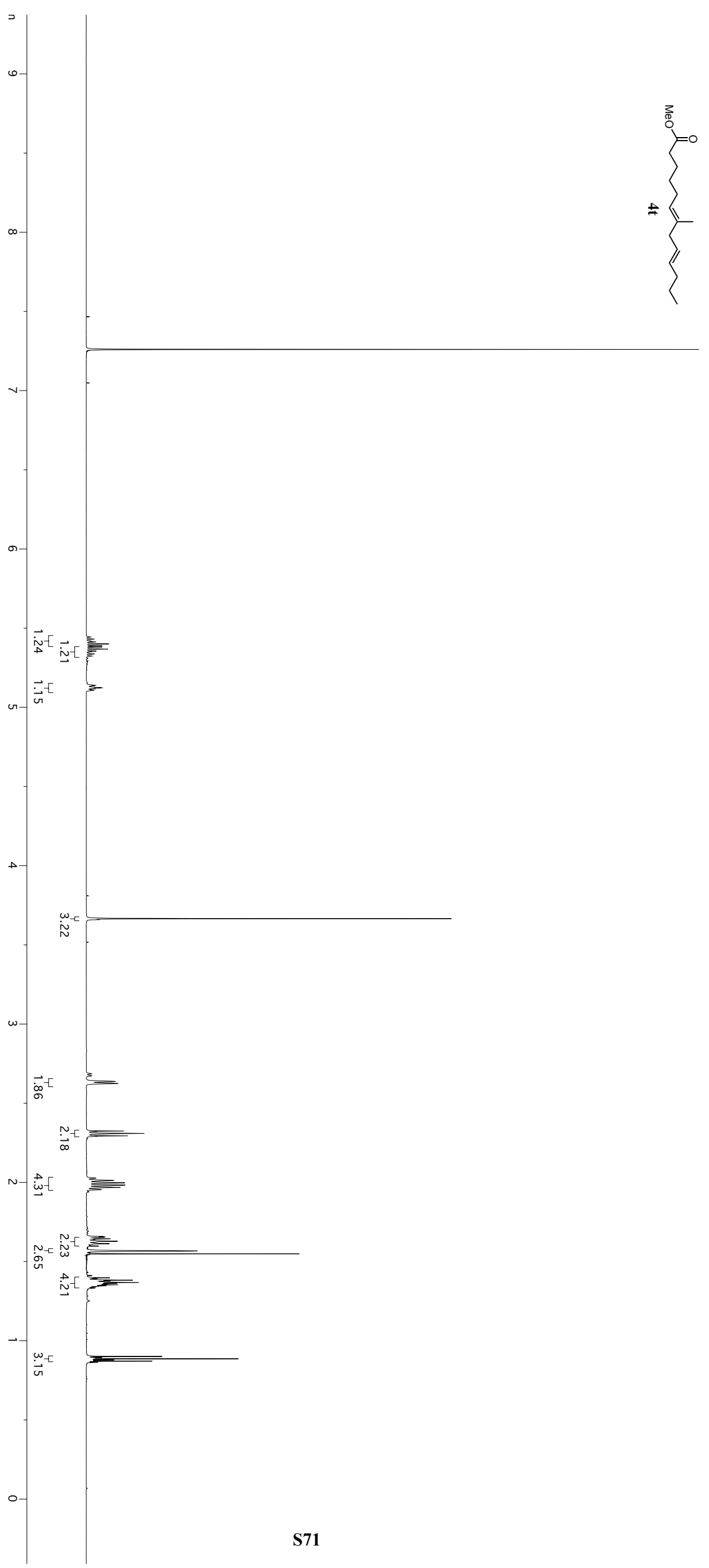




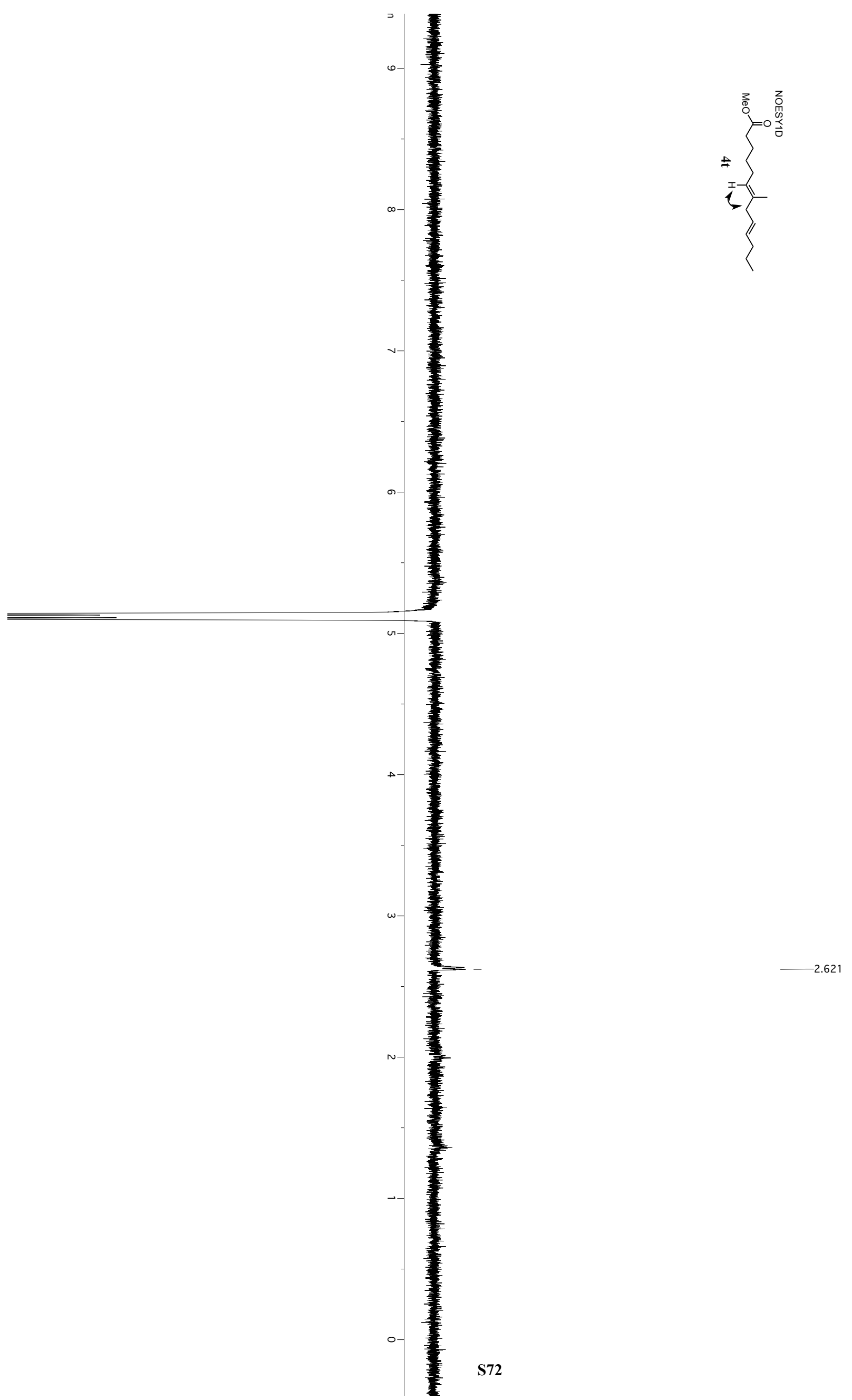




$$
\mid
$$



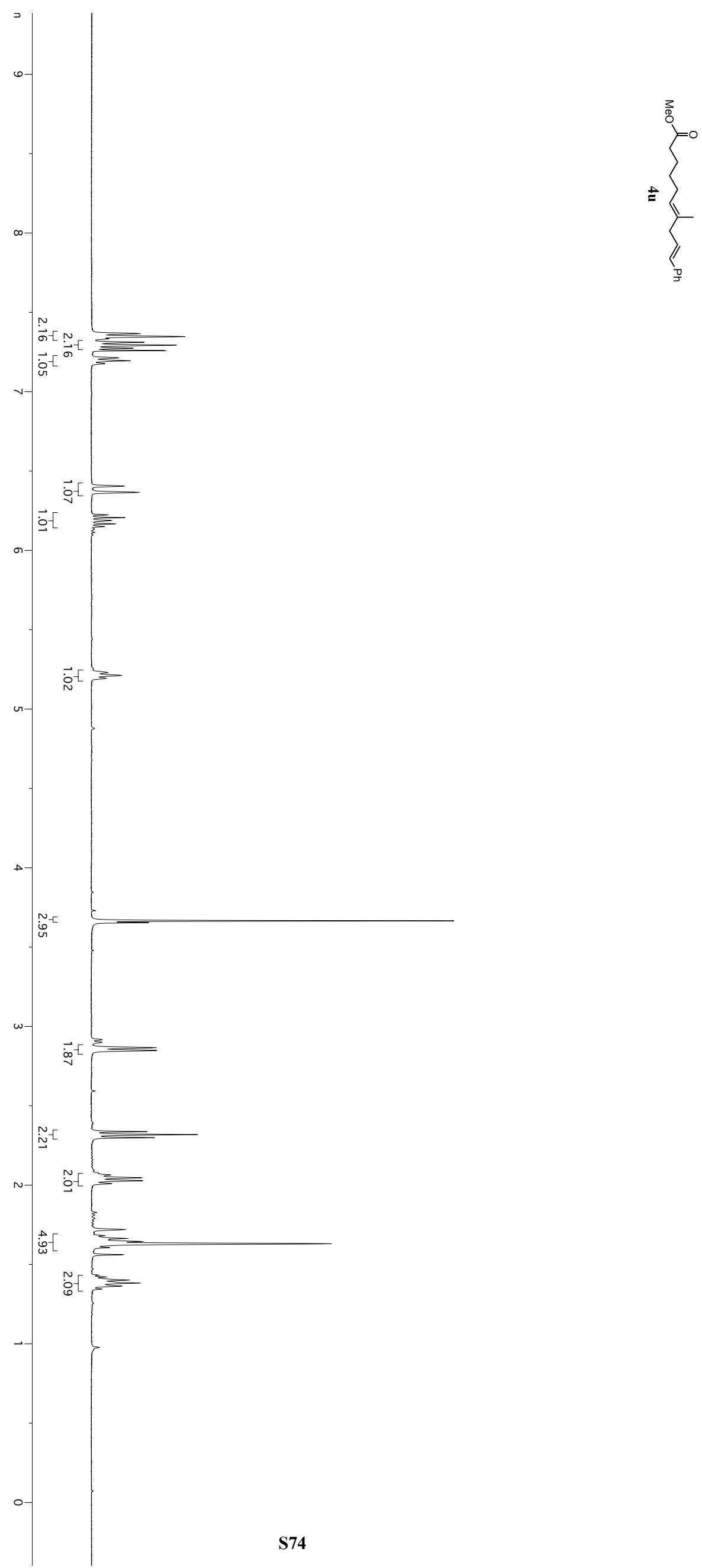


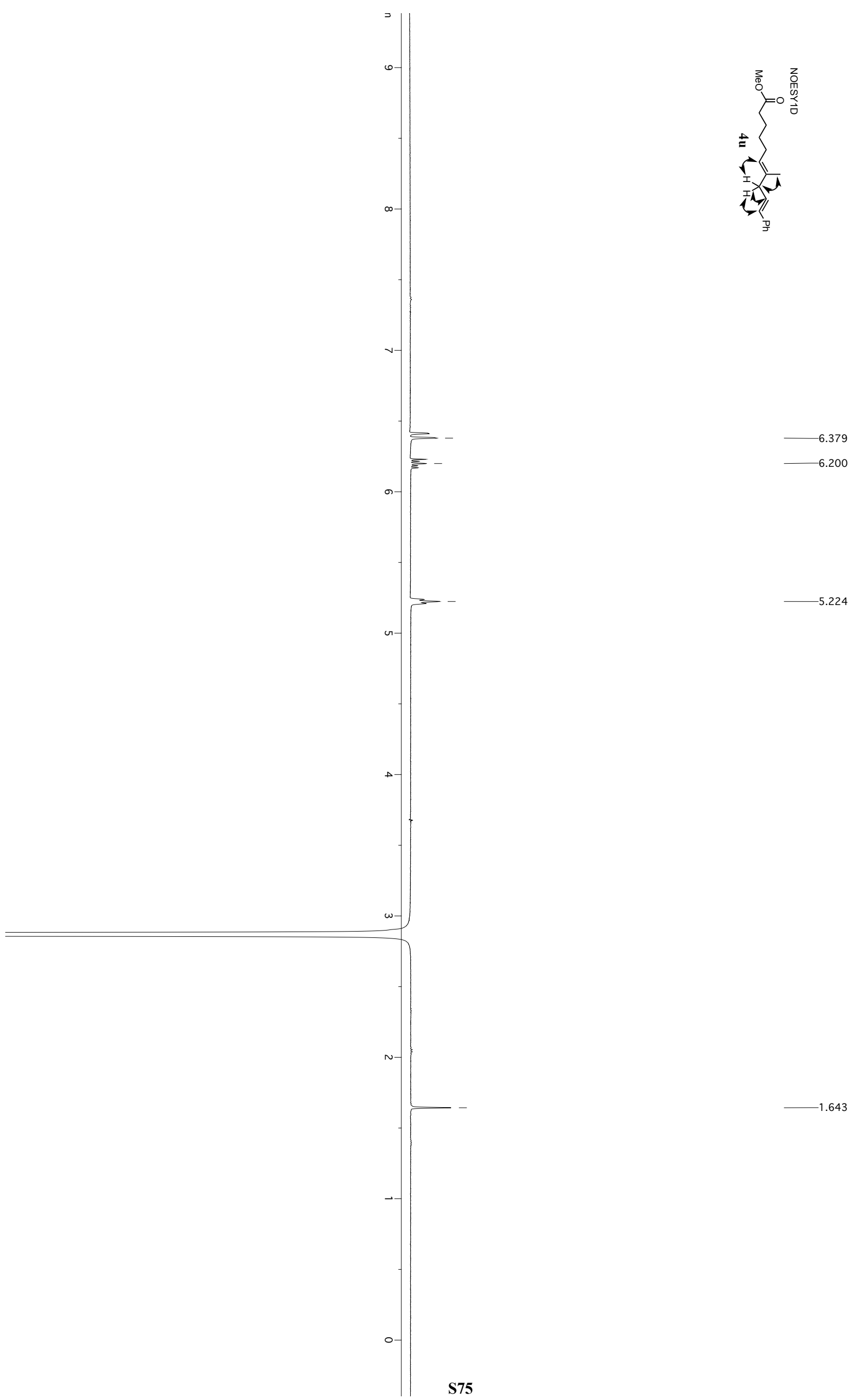




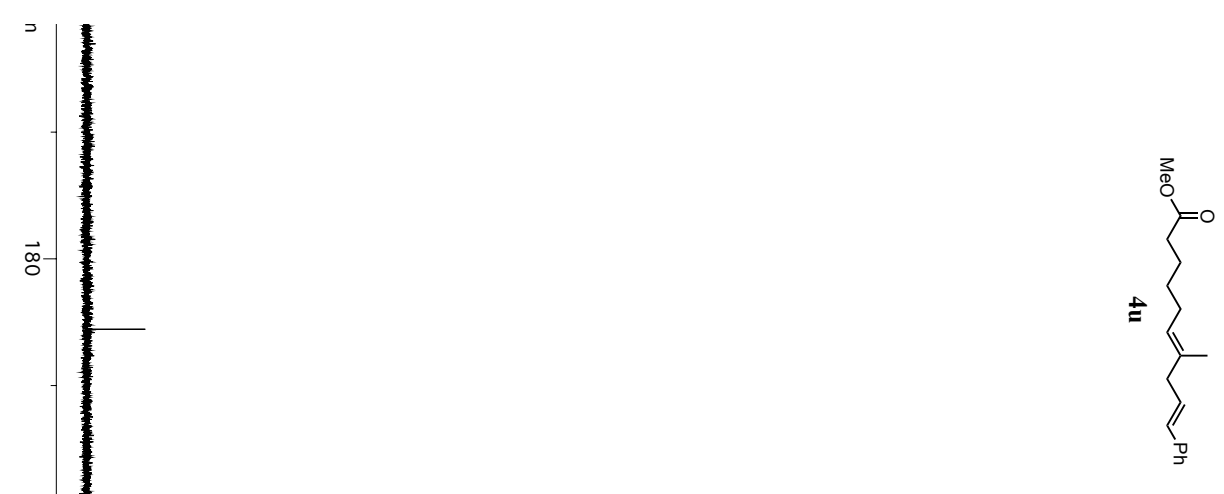




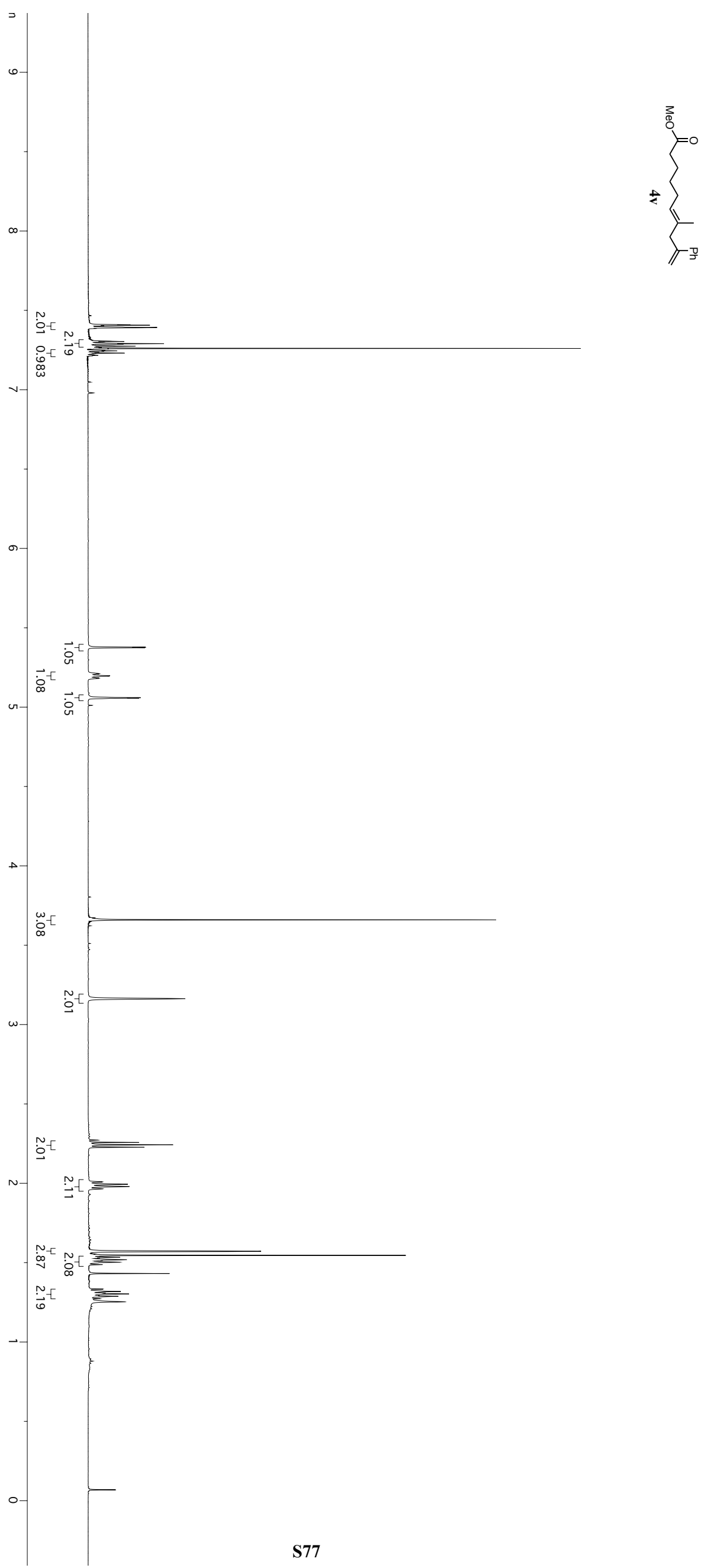




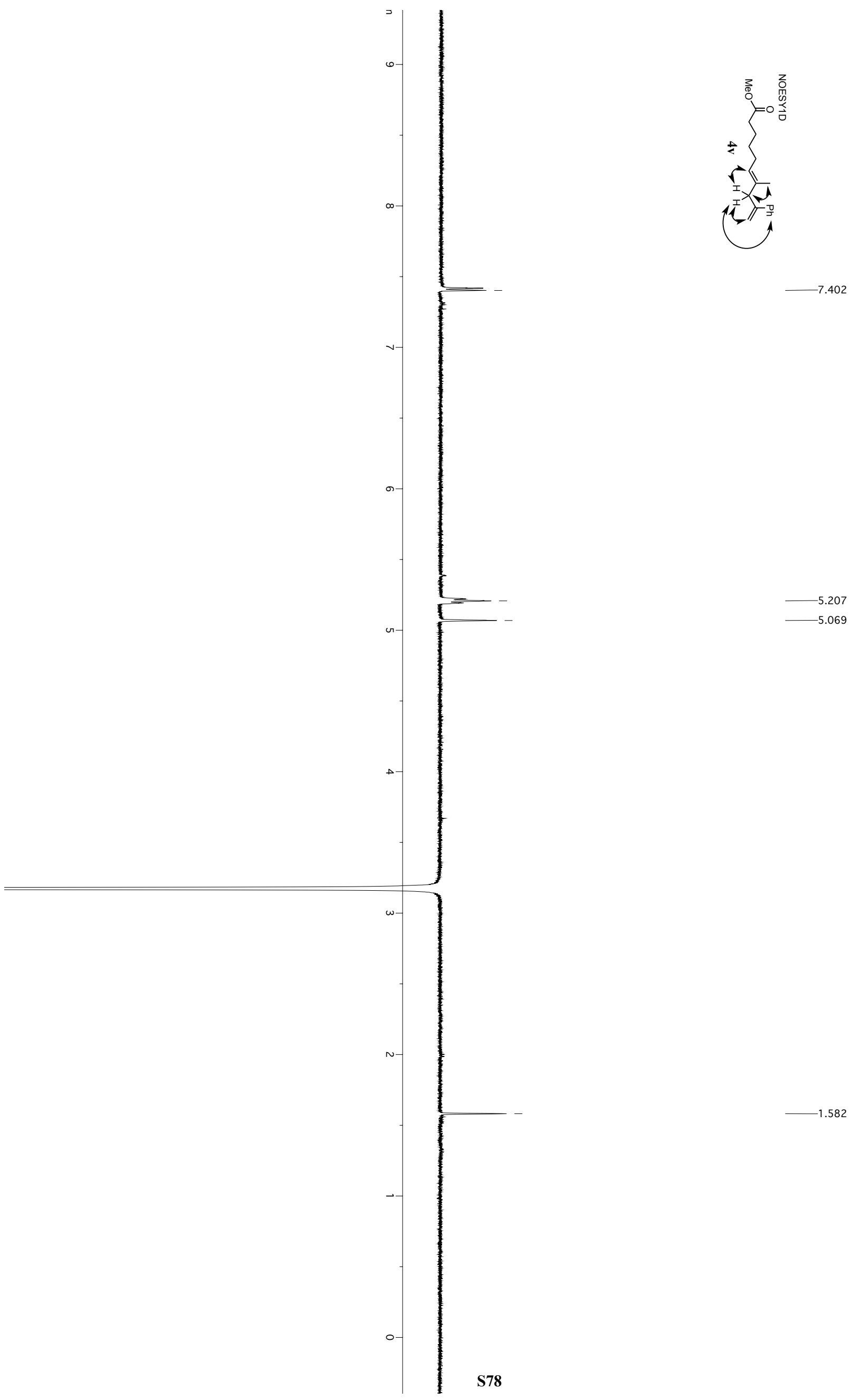




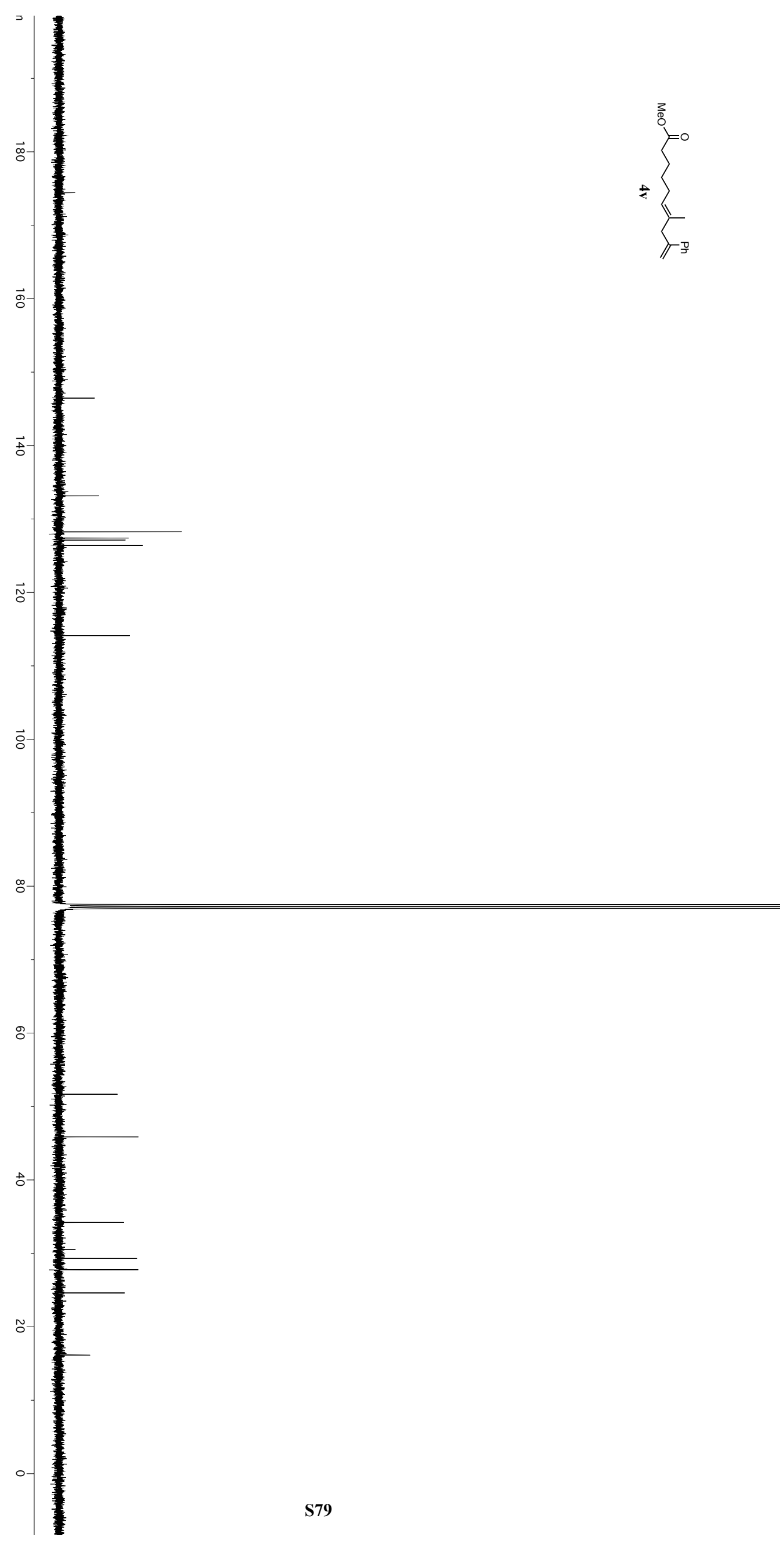

